Preprint typeset in JHEP style - HYPER VERSION

\title{
BPS spectra and 3-manifold invariants
}

\author{
Sergei Gukov ${ }^{1}$, Du Pei ${ }^{1,2}$, Pavel Putrov ${ }^{3}$, Cumrun Vafa ${ }^{4}$ \\ 1 Walter Burke Institute for Theoretical Physics, California Institute of Technology, \\ Pasadena, CA 91125, USA \\ 2 Center for Quantum Geometry of Moduli Spaces, Deparment of Mathematics, University \\ of Aarhus, DK-8000, Denmark \\ ${ }^{3}$ School of Natural Sciences, Institute for Advanced Study, Princeton, NJ 08540, USA \\ 4 Jefferson Physical Laboratory, Harvard University, Cambridge, MA 02138, USA
}

\begin{abstract}
We provide a physical definition of new homological invariants $\mathcal{H}_{a}\left(M_{3}\right)$ of 3 manifolds (possibly, with knots) labeled by abelian flat connections. The physical system in question involves a $6 \mathrm{~d}$ fivebrane theory on $M_{3}$ times a 2-disk, $D^{2}$, whose Hilbert space of BPS states plays the role of a basic building block in categorification of various partition functions of $3 \mathrm{~d} \mathcal{N}=2$ theory $T\left[M_{3}\right]: D^{2} \times S^{1}$ half-index, $S^{2} \times S^{1}$ superconformal index, and $S^{2} \times S^{1}$ topologically twisted index. The first partition function is labeled by a choice of boundary condition and provides a refinement of Chern-Simons (WRT) invariant. A linear combination of them in the unrefined limit gives the analytically continued WRT invariant of $M_{3}$. The last two can be factorized into the product of half-indices. We show how this works explicitly for many examples, including Lens spaces, circle fibrations over Riemann surfaces, and plumbed 3-manifolds.
\end{abstract}

CALT-TH-2016-039 


\section{Contents}

1. Introduction 2

2. Fivebranes on 3-manifolds and categorification of WRT invariant 5

2.1 Preliminaries 5

$2.2 D^{2} \times S^{1}$ partition function of $T\left[M_{3}\right]$ and WRT invariant 5

2.3 Superconformal index of $T\left[M_{3}\right]$ and its factorization 11

2.4 Further refinement $\quad 12$

2.5 Topologically twisted index of $T\left[M_{3}\right] \quad 12$

2.6 Generalization to $U(N) \quad 13$

2.7 Relation to open GW invariants on $T^{*} M_{3} \quad 14$

2.8 3-manifolds and the "bottom row" 16

3. Examples $\quad 19$

$3.1 M_{3}=S^{3} \quad 19$

3.1.1 Categorifying the index 21

3.1.2 The $t=q^{\beta}$ reduction from BPS spectral sequence 22

$3.2 M_{3}=L(p, 1) \quad 24$

3.2.1 Refined superconformal index 24

3.2.2 Topologically twisted index of $T[L(p, 1)] \quad 26$

$\begin{array}{ll}3.2 .3 t=q^{\beta} \text { reduction } & 29\end{array}$

3.2.4 Cyclotomic expansion 31

3.2.5 Positivity of coefficients 33

3.2.6 Comparison with refined CS 35

$3.3 M_{3}=O(-p) \rightarrow \Sigma_{g}$

3.3.1 Homological blocks and WRT invariant 39

3.3.2 Factorization of the superconformal index 40

3.3.3 Comparison with refined CS 42

$3.4 M_{3}=$ plumbed $\quad 43$

3.4.1 Homological blocks $\widehat{Z}_{a} \quad 44$

3.4.2 Problems on the $T\left[M_{3}\right]$ side $\quad 47$

4. Adding knots and links into $M_{3} \quad 47$

4.1 Links in $M_{3}$ and line operators in $T\left[M_{3}\right] \quad 48$

4.2 Links in $S^{2} \times S^{1}$ and Rozansky's proposal $\quad 52$

4.3 Links in $L(p, 1) \quad 55$

4.3.1 Unrefined index of $T[L(p, 1)]$ with Wilson loops 56

$\begin{array}{lll}\text { 4.3.2 Refinement and categorification } & 57\end{array}$

$\begin{array}{lll}4.4 & \text { Comparison with refined CS } & 58\end{array}$ 
B. Cabling in $M_{3}$ versus color in $T\left[M_{3}\right]$

\section{Introduction}

Can we hear the shape of a drum? Much like harmonics of a musical instrument, spectra of quantum systems contain wealth of useful information. Of particular interest are supersymmetric or the so-called BPS states which, depending on the problem at hand, can manifest themselves either as minimal surfaces, or solutions to partial differential equations, or other "extremal" objects. Thus, a spectrum of BPS states in Calabi-Yau compactifications can be used to reconstruct the geometry of the Calabi-Yau space itself and, as we explain in this paper, spectra of BPS states play a similar role in low-dimensional topology.

The old approach to constructing numerical invariants of 3- and 4-manifolds, as well as the corresponding homological invariants of 3-manifolds, is based on gauge theory. The famous examples are Donaldson-Witten and Seiberg-Witten (SW) invariants of 4- and 3-manifolds, and corresponding instanton and monopole [1] Floer homologies of 3-manifolds. All of them were extensively studied in mathematical literature and have appropriate rigorous definitions that go back to the previous century. The numerical invariants are realized in terms of counting solutions to certain partial differential equations, while the homological invariants build on the ideas of Andreas Floer [2]. In particular, Seiberg-Witten invariants of 4-manifolds had great success distinguishing some homeomorphic but non-diffeomorphic 4-manifolds. And, in the world of 3-manifolds, the so-called Heegaard Floer homology constructed by Ozsvath and Szabo [3] gives a much simpler and non-gauge theoretic definition of a homological invariant, which is believed to be equivalent to the monopole Floer homology.

A seemingly different class of 3-manifold invariants, the so-called Witten-ReshetikhinTuraev (WRT) invariants [4,5], comes from a different type of TQFT, which sometimes is called "of Schwarz type" to distinguish it from the TQFTs "of cohomological type" mentioned in the previous paragraph [6]. At the turn of the century, however, the distinction between the two types started to blur and the ideas of the present paper suggest it may even go away completely in the future. In fact, the first hints for this go back to the early work [7-10] that relates Seiberg-Witten theory in three dimensions to Chern-Simons theory with $U(1 \mid 1)$ super gauge group. The latter provides a much simpler invariant compared to the usual Chern-Simons theory, say, with $S U(2)$ gauge group, due to cancelations between bosonic and fermionic contributions. Therefore, if one can find a 4d TQFT that categorifies $S U(2)$ Chern-Simons theory in 3d, similar to how 4d SW theory categorifies 3d SW theory, it would help a great deal with the classification problem of smooth 4 -manifolds. The first step in 
constructing such categorification is, of course, to find a homological invariant of 3-manifolds whose (equivariant) Euler characteristic gives the WRT invariant.

The existence of such 4d TQFT was envisioned by Crane and Frenkel [11] more than 20 years ago, and the first evidence came with the advent of knot homology [12-14] which categorifies WRT invariants of knots and links (realized by Wilson lines in CS theory) in $S^{3}$, also known as the colored Jones polynomial. The physical understanding of a homological approach to HOMFLY polynomials was independently initiated in the physics literature in [15], which later led to the physical interpretation of Khovanov-Rozansky homology as certain BPS Hilbert spaces [16-19] (see e.g. [20,21] for an overview and an extensive list of references).

The physical construction suggests that there should be a homological invariant that categorifies (in a certain sense) the WRT invariant of general 3-manifolds with knots inside. Namely, such homological invariant can be understood as the BPS sector of the Hilbert space of the $6 \mathrm{~d} \mathcal{N}=(2,0)$ theory (that is a theory describing dynamics of coincident M5-branes in M-theory) on $M_{3} \times D^{2} \times \mathbb{R}$ with a certain supersymmetry preserving background along $M_{3} \times D^{2}$. Equivalently, if one first reduces the $6 \mathrm{~d}$ theory on $M_{3}$, it can be understood as the BPS Hilbert space of the effective $3 \mathrm{~d} \mathcal{N}=2$ theory $T\left[M_{3}\right]$ on $D^{2} \times \mathbb{R}$. On the other hand, if one first compactifies on $D^{2}$, one does not get an ordinary 4 d gauge theory on $M_{3} \times \mathbb{R}$ like 4d SW gauge theory ${ }^{1}$ :

space of BPS states $\mathcal{H}_{\mathrm{BPS}}$

of $3 \mathrm{~d} \mathcal{N}=2$ theory $T\left[M_{3}\right]$ on $D^{2}$
6 d $(2,0)$ theory on $\mathbb{R} \times D^{2} \times M_{3}$

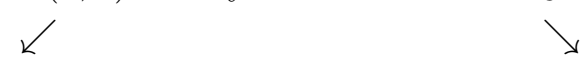

$$
\begin{gathered}
\text { Hilbert space } \mathcal{H}_{M_{3}} \text { of } \\
\text { 4d "Crane-Frenkel TQFT" } \\
\text { on } M_{3}
\end{gathered}
$$

There is another natural SUSY-preserving background on which one can quantize $T\left[M_{3}\right]$. Since the IR physics of $T\left[M_{3}\right]$ is governed by a non-trivial $3 \mathrm{~d} \mathcal{N}=2 \mathrm{SCFT}$, one can consider its radial quantization and study its Hilbert space on $S^{2} \times \mathbb{R}$. This should provide us with another non-trivial homological invariant of $M_{3}$ which should have roughly the same level of complexity as the BPS Hilbert space on $D^{2} \times \mathbb{R}$ which categorifies the WRT invariant, but with several advantages due to the presence of operator-state correspondence and no need to specify a boundary condition at $\partial D^{2}=S^{1}$.

The set of boundary conditions that one can put at $\partial D^{2} \cong S^{1}$ in the path integral can be understood as follows. Let us represent $D^{2}$ as an elongated cigar, which asymptoically looks like $S^{1} \times \mathbb{R}$. After compactification of the stack of fivebranes on $S^{1}$ we obtain $5 \mathrm{~d}$ maximally supersymmetric gauge theory. The supersymmetric vacua of such theory on $M_{3} \times \mathbb{R}$ (where $\mathbb{R}$ is the original time direction) are given by flat connections ${ }^{2}$ on $M_{3}$. The number of such

\footnotetext{
${ }^{1}$ Roughly speaking, the effective $4 \mathrm{~d}$ theory is an infinite KK-like tower on $4 \mathrm{~d}$ gauge theories. However one needs to appropriately sum it up. The decategorified counterpart of such summation was studied in [22].

${ }^{2}$ The choice of such flat connections should not be confused with the choice of flat connections in CS theory on $M_{3}$. As will be explained later in detail they are related by S-transform.
} 
supersymetric vacua is the same as the number of boundary conditions ( $c f .[18,23-25])$. As we will see later, the subset of such boundary conditions that corresponds to abelian flat connections plays important role.

In this paper we study the relation between such homological invariants and their decategorified counterparts - superconformal indices. The structure described above, of course, will also manifest itself at the level of partition functions, i.e. indices, once we compactify the time on $S^{1}$. For some of the examples in this paper for there are no cancellations among states in computing the refined index. In those cases, the homological invariants are faithfully captured by the refined index computation.

The organization of the paper is as follows. In Section 2 we review some results of [26] and summarize general conjectures about homological invariants of closed 3-manifolds mentioned here and their decategorified versions. In Section 3 we consider various examples for which we explicitly verify these conjectures. In Section 4 we extend it to the case of 3-manifolds with knots. Note, the main part of Section 4 is written in jargonish shorthand, using the concepts and notations introduced earlier. Various details and generalizations of this work can be found in the appendices. Thus, Appendix A explains how the WRT invariants of general negative definite plumbed 3-manifolds can be analytically continued away from roots of unity to produce power series in $q$ with integer powers and integer coefficients, required for categorification. In Appendix B, we compare the ordinary Khovanov homology of the $n$-th cabling of the unknot in a 3 -sphere to the refined partition function of $3 \mathrm{~d} \mathcal{N}=2$ theory $T\left[S^{3}\right]$ in the presence of line operators, $c f$. Figure 1. Finally, in Appendix C we explain how categorification of the index of $T\left[M_{3}\right]$ relates to categorification of the Turaev-Viro invariants.

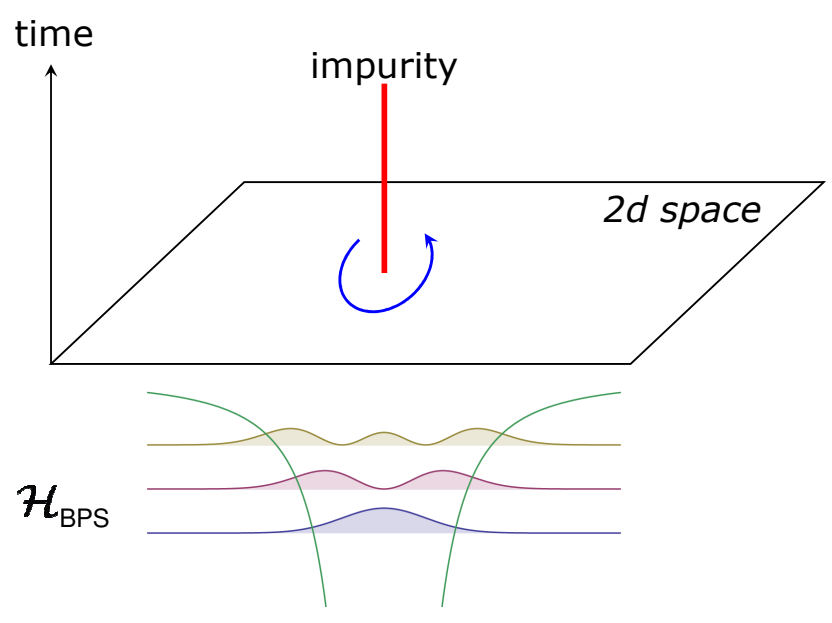

Figure 1: The space of BPS states in $3 \mathrm{~d} \mathcal{N}=2$ theory on $\mathbb{R} \times D^{2}$ with an impurity, relevant to the physical realization of Heegaard Floer homology $H F\left(M_{3}\right)$, monopole Floer homology $H M\left(M_{3}\right)$, as well as categorification of WRT invariants of 3-manifolds with knots. 


\section{Fivebranes on 3-manifolds and categorification of WRT invariant}

The goal of this section is to introduce the key players and their interrelation. To keep the discussion simple and concrete, we choose the gauge group to be $G=S U(2)$ for most of it, and then in section 2.6 briefly comment how everything can be generalized to higher ranks.

\subsection{Preliminaries}

Before we present a mathematically-friendly summary of our proposal and the physics behind it, we need to introduce some notations, especially those relevant to abelian flat connections that will be central in our discussion.

Consider a closed and connected 3-manifold $M_{3}$, with $\partial M_{3}=\emptyset$. In order to present the results in full generality, it will be useful to consider the linking pairing on the torsion part of $H_{1}\left(M_{3}, \mathbb{Z}\right)$ :

$$
\begin{aligned}
\ell k: \text { Tor } H_{1}\left(M_{3}\right) \otimes \operatorname{Tor} H_{1}\left(M_{3}\right) & \longrightarrow \mathbb{Q} / \mathbb{Z} \\
{[a] \otimes[b] } & \longmapsto \#\left(a \cap b^{\prime}\right) / n
\end{aligned}
$$

where $b^{\prime}$ is a 2-chain such that $\partial b^{\prime}=n b$ for an integer $n$. Such $b^{\prime}$ and $n$ exist because $[b]$ is torsion. As usual, \# $\left(a \cap b^{\prime}\right)$ denotes the number of intersection points counted with signs determined by the orientation. Note that the linking form provides an isomorphism between Tor $H_{1}\left(M_{3}\right)$ and its Pontryagin dual (Tor $\left.H_{1}\left(M_{3}\right)\right)^{*} \equiv \operatorname{Hom}\left(\operatorname{Tor} H_{1}\left(M_{3}\right), U(1)\right)$ via the pairing $e^{2 \pi i \ell k(\cdot, \cdot)}$.

The $\mathbb{Z}_{2}$ Weyl group acts on the elements $a \in$ Tor $H_{1}\left(M_{3}, \mathbb{Z}\right)$ via $a \mapsto-a$. The set of orbits is the set of connected components of abelian flat $S U(2)$ connections on $M_{3}$ (i.e., connections in the image of $\rho: \mathcal{M}_{\text {flat }}\left(M_{3}, U(1)\right) \rightarrow \mathcal{M}_{\text {flat }}\left(M_{3}, S U(2)\right)$ from the embedding $U(1) \subset S U(2)){ }^{3}$

$$
\text { Tor } H_{1}\left(M_{3}, \mathbb{Z}\right) / \mathbb{Z}_{2} \cong \pi_{0} \mathcal{M}_{\text {flat }}^{\text {ab }}\left(M_{3}, S U(2)\right) \text {. }
$$

It is also useful to introduce a shorthand notation for the stabilizer subgroup:

$$
\mathcal{W}_{a} \equiv \operatorname{Stab}_{\mathbb{Z}_{2}}(a)=\left\{\begin{array}{c}
\mathbb{Z}_{2}, a=-a \\
1, \text { otherwise }
\end{array}\right.
$$

\section{2 $D^{2} \times S^{1}$ partition function of $T\left[M_{3}\right]$ and WRT invariant}

Now we are ready to present a slightly generalized and improved version of the results from $[26$, sec. 6].

\section{Categorification of WRT invaraiant}

Let $Z_{S U(2)_{k}}\left[M_{3}\right]$ be the partition function of $S U(2)$ Chern-Simons theory with "bare" level $(k-2)$ on $M_{3}$, also known as the WRT invariant. We use the standard "physics" normalization where

$$
Z_{S U(2)_{k}}\left[S^{2} \times S^{1}\right]=1
$$

\footnotetext{
${ }^{3}$ It is in fact $\left(\text { Tor } H_{1}\left(M_{3}, \mathbb{Z}\right)\right)^{*} / \mathbb{Z}_{2}$ that is canonically identified with components of abelian flat connections. However, as the distinction between Tor $H_{1}\left(M_{3}, \mathbb{Z}\right)$ and its dual is only important in section 2.2 , we will use the same set of labels $\{a, b, \ldots\}$ for elements in both groups.
} 
and

$$
Z_{S U(2)_{k}}\left[S^{3}\right]=\sqrt{\frac{2}{k}} \sin \left(\frac{\pi}{k}\right) .
$$

The following conjecture was proposed in $[26]{ }^{4}$

Conjecture 2.1 The WRT invariant can be decomposed into the following form:

$$
Z_{S U(2)_{k}}\left[M_{3}\right]=\left.(i \sqrt{2 k})^{b_{1}\left(M_{3}\right)-1} \sum_{\substack{a, b \in \\ \text { Tor } H_{1}\left(M_{3}, \mathbb{Z}\right) / \mathbb{Z}_{2}}} e^{2 \pi i k \ell k(a, a)} S_{a b} \widehat{Z}_{b}(q)\right|_{q \rightarrow e^{\frac{2 \pi i}{k}}}
$$

with $^{56}$

$$
\widehat{Z}_{b}(q) \in 2^{-c} q^{\Delta_{b}} \mathbb{Z}[[q]] \quad \Delta_{b} \in \mathbb{Q}, \quad c \in \mathbb{Z}_{+}
$$

convergent in $|q|<1$ and

$$
S_{a b}=\frac{e^{4 \pi i \ell k(a, b)}+e^{-4 \pi i \ell k(a, b)}}{\left|\mathcal{W}_{a}\right| \sqrt{\left|\operatorname{Tor} H_{1}\left(M_{3}, \mathbb{Z}\right)\right|}}
$$

In other words, we claim the existence of new 3 -manifold invariants $\widehat{Z}_{a}$, which admit $q$-series expansion with integer powers and integer coefficients (hence, more suitable for categorification) and from which the WRT invariant can be reconstructed via (2.6). While the formal mathematical definition of the invariants $\widehat{Z}_{a}$ is waiting to be discovered, they admit a physics definition that will be reviewed below and can be independently computed via techniques of resurgent analysis. In particular, each term

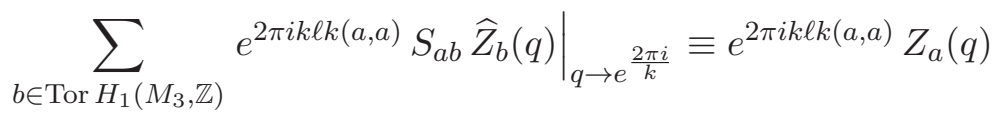

in the sum (2.6) is a certain resummation of the perturbative (in $\frac{2 \pi i}{k}$ or, equivalently, in $(1-q))$ expansions of the WRT invariant around the corresponding abelian flat connection $a[22]$.

In order to avoid unnecessary technical complications, in the rest of this paper we assume that Tor $H_{1}\left(M_{3}, \mathbb{Z}\right)$ has no $\mathbb{Z}_{2}$ factors. ${ }^{7}$ Under this assumption, the $S$-matrix satisfies

$$
\sum_{b} S_{a b} S_{b c}=\delta_{a c}
$$

\footnotetext{
${ }^{4} \mathrm{~A}$ related conjecture was made in [27]. However it did not include the $S$-transform, which is crucial for restoring integrality and categorification.

${ }^{5}$ The constant positive integer $c$ depends only on $M_{3}$ and in a certain sense measures its "complexity". In many simple examples $c=0$, and the reader is welcome to ignore $2^{-c}$ factor which arises from some technical subtleties. Its physical origin will be explained later in the paper.

${ }^{6}$ Later in the text we will sometimes use slightly redefined quantities, $\widehat{Z}_{a}(q) \rightarrow q^{\Delta} \widehat{Z}_{a}(q)$, where $\Delta$ is a common, $a$ independent rational number.

${ }^{7}$ Recall that Tor $H_{1}\left(M_{3}, \mathbb{Z}\right)$, as a finitely generated abelian group, can be decomposed into Tor $H_{1}\left(M_{3}, \mathbb{Z}\right)=$ $\prod_{i} \mathbb{Z}_{p_{i}}$. We ask for a fairly weak condition that $\mathbb{Z}_{2}$ doesn't appear in this decomposition. In other words, $M_{3}$ is a $\mathbb{Z}_{2}$-homology sphere. Equivalently, there is a unique Spin structure on $M_{3}$, so that there is no ambiguity in specifying Nahm-pole boundary condition for $\mathcal{N}=4 S U(2) \mathrm{SYM}$ on $M_{3} \times \mathbb{R}_{+}[18]$. The general case, in principle, could also be worked out. We leave it as an exercise to an interested reader.
} 
Moreover, as will be discussed in detail below, physics predicts the existence of a $\mathbb{Z} \times \mathbb{Z} \times$ Tor $H_{1}\left(M_{3}, \mathbb{Z}\right) / \mathbb{Z}_{2}$ graded homological invariant of $M_{3}$ :

$$
\mathcal{H}_{D^{2}}\left[M_{3}\right]=\bigoplus_{a \in \operatorname{Tor}} \bigoplus_{1}\left(M_{3}, \mathbb{Z}\right) / \mathbb{Z}_{2} \mathcal{H}_{a}\left[M_{3}\right], \quad \mathcal{H}_{a}\left[M_{3}\right]=\bigoplus_{\substack{i \in \mathbb{Z}+\Delta a \\ j \in \mathbb{Z}}} \mathcal{H}_{a}^{i, j}
$$

which categorifies the $q$-series $\widehat{Z}_{a}(q)$. Namely,

$$
\widehat{Z}_{a}(q)=2^{-c} \sum_{\substack{i \in \mathbb{Z}+\Delta_{a} \\ j \in \mathbb{Z}}} q^{i}(-1)^{j} \operatorname{dim} \mathcal{H}_{a}^{i, j} .
$$

Because of their close relation to homological invariants, we usually refer to $\widehat{Z}_{a}(q)$ as homological blocks. The vector space $\mathcal{H}_{D^{2}}\left[M_{3}\right]$ can be interpreted as the closed 3-manifold analog of Khovanov-Rozansky knot homology. From this point of view, $e^{4 \pi i \ell k(a, \cdot)}$ can be understood as the variable associated with Tor $H_{1}\left(M_{3}, \mathbb{Z}\right) / \mathbb{Z}_{2}$ grading and enters into the decomposition (2.6) much like $q$, the variable for one of the $\mathbb{Z}$-gradings (the " $q$-grading"). Note that the label $a$ in $\mathcal{H}_{a}^{i, j}$ is reminiscent of the $\operatorname{Spin}^{c}$ structure in Heegaard/monopole Floer homologies of 3-manifolds. This fact, of course, is not an accident and plays an important role in the relation between Heegaard/monopole Floer homologies of 3-manifolds and the categorification of WRT invariants [26].

Next, we describe the physics behind the Conjecture 2.1. (A mathematically inclined reader may skip directly to Conjecture 2.2.)

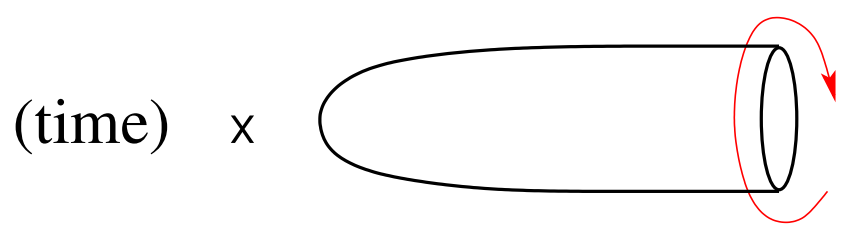

Figure 2: Another representation of the background in Figure 1.

\section{Physics behind the proposal}

From physics point of view, the homological invariants $\mathcal{H}_{a}\left[M_{3}\right]$ can be realized by the following M-theory geometry,

$$
\begin{array}{ccccc}
N \text { fivebranes: } & \mathbb{R} \times & M_{3} & \times & D^{2} \\
& & \cap & & \cap \\
\text { space-time: } & \mathbb{R} \times & T^{*} M_{3} & \times & T N \\
& & \circlearrowright & & \circlearrowright \\
\text { symmetries: } & & \text { "U } U(1)_{N} " & & U(1)_{q} \times U(1)_{R} .
\end{array}
$$

or, equivalently, any of its dual descriptions (some of which will be discussed below). Here, the first two lines summarize the geometry of the fivebranes and their ambient space, whereas 
the last line describes their symmetries. The reason " $U(1)_{N}$ " appears in quotes is that it is a symmetry of our physical system only when $M_{3}$ is a Seifert 3-manifold, unlike the "universal" symmetry group $U(1)_{q} \times U(1)_{R}$.

In order to preserve supersymmetry for a general metric on $M_{3}$, it has to be embedded in the geometry of ambient space-time as a supersymmetric (special Lagrangian) cycle $M_{3} \subset C Y_{3}$ which, according to McLean's theorem, always looks like $M_{3} \subset T^{*} M_{3}$ near the zero section. Equivalently, the geometry $T^{*} M_{3}$ represents a partial topological twist on the fivebrane worldvolume, upon which three of the scalar fields on the world-volume become sections of the cotangent bundle of $M_{3}$. As a result, one can first reduce the $6 \mathrm{~d}(2,0)$ theory - the worldvolume theory of M5-branes - on $M_{3}$ to obtain a $3 \mathrm{~d} \mathcal{N}=2$ SCFT usually denoted as $T\left[M_{3} ; G\right]$, where $G=U(N)$ or $S U(N), N$ being the number of M5 branes. All SUSYprotected objects like partition functions, index and BPS spectra of the resulting theory $T\left[M_{3} ; G\right]$ do not depend ${ }^{8}$ on the metric of $M_{3}$, and give rise to numerical as well as homological invariants of $M_{3}$.

Similarly, in order to preserve supersymmetry of the brane system (2.13) along the other world-volume directions of the fivebranes, one needs to introduce a SUSY-preserving background along $D^{2}$. Moreover, it needs to be done in a way that preserves the rotation symmetry $U(1)_{q} \times U(1)_{R}$ and allows to keep track of the corresponding quantum numbers (spins) of BPS states, as required for categorification. The suitable background can be described in a number of equivalent ways: as the Omega-background along $T N \cong \mathbb{R}_{q, t}^{4}$ in which $D^{2} \cong \mathbb{R}^{2}$ is embedded as a linear subspace, or as a $U(1)_{q} \times U(1)_{R}$ invariant Lagrangian submanifold (the "cigar") $D^{2}$ in the Taub-NUT space $T N$ where one keeps track of the spin with respect to the rotation symmetry, $c f$. Figure 2. To emphasize that one keeps track of both spins under $U(1)_{q} \times U(1)_{R}$ symmetry, the adjective refined is often added to the invariant, BPS state, or other object under consideration.

When $M_{3}$ is a Seifert manifold, the brane system (2.13) enjoys an extra symmetry $U(1)_{N}$ that appears in a degeneration limit of the metric on $M_{3}$ and can be used to redefine the R-symmetry of the SCFT $T\left[M_{3}\right]$. When $M_{3}$ is $\Sigma \times S^{1}$, the symmetry $U(1)_{N} \times U(1)_{R}$ is further enhanced to the $S U(2)_{N} \times S U(2)_{R}$ R-symmetry of the $3 \mathrm{~d} \mathcal{N}=4$ theory $T\left[\Sigma \times S^{1}\right]$; when $M_{3}$ is a generic Seifert manifold, one combination gives the R-symmetry of the $3 \mathrm{~d} \mathcal{N}=2$ theory $T\left[M_{3}\right]$ which we denote as $U(1)_{R}$, while another is a flavor symmetry $U(1)_{\beta}$, see $[26$, sec. 3.4] for details.

After reduction on $M_{3}$, the system $(2.13)$ gives a theory $T\left[M_{3} ; G\right]$ in space-time $D^{2} \times \mathbb{R}$, illustrated in Figure 1, and we can consider its Hilbert space with a certain boundary condition at $\partial D^{2}=S^{1}$. For $N=2$ and $G=S U(2)$ — the case that we will be mostly considering in this paper — these boundary conditions turn out to be labeled by $a \in \operatorname{Tor} H_{1}\left(M_{3}, \mathbb{Z}\right) / \mathbb{Z}_{2}$.

${ }^{8} \mathrm{~A}$ "folk theorem" states that any continuous deformation of the metric on $M_{3}$ results in a $Q$-exact term of the supergravity background. However, there may be dependence on discrete data such as the Atiyah 2 -framing [28]. 
Then, we arrive at a set of doubly-graded homological invariants of $M_{3}$ labeled by $a$,

$$
\mathcal{H}_{a}\left[M_{3}\right]=\mathcal{H}_{T\left[M_{3}\right]}\left(D^{2} ; a\right)=\bigoplus_{\substack{i \in \mathbb{Z}+\Delta_{a}, j \in \mathbb{Z}}} \mathcal{H}_{a}^{i, j},
$$

given by the BPS sector of the Hilbert space of $T\left[M_{3} ; S U(2)\right]$. This is the subspace annihilated by two of the four supercharges of the $3 \mathrm{~d} \mathcal{N}=2$ supersymmetry (different choices are related by automorphisms of the superconformal algebra, resulting in isomorphic BPS spaces). The grading $i$ counts the charge under the $U(1)_{q}$ rotation of $D^{2}$ and the "homological" grading $j$ corresponds to R-charge of the $U(1)_{R}$ R-symmetry. When $M_{3}$ is Seifert, the $U(1)_{\beta}$ symmetry will give rise to the third grading ${ }^{9}$ on $\mathcal{H}_{a}\left[M_{3}\right]$.

One can understand $\mathcal{H}_{a}\left[M_{3}\right]=\mathcal{H}_{T\left[M_{3}\right]}\left(D^{2} ; a\right)$ as the massless multi-particle BPS spectrum of $T\left[M_{3}\right]$ with a label $a \in$ Tor $H_{1}\left(M_{3}, \mathbb{Z}\right) / \mathbb{Z}_{2}$ being a discrete charge. From M-theory point of view, the BPS particles of $T\left[M_{3}\right]$ arise from M2-branes ending on the pair of M5branes that realize the $A_{1} 6 \mathrm{~d} \mathcal{N}=(2,0)$ theory. The boundaries of M2-branes wrap 1-cycles $(\widetilde{a},-\widetilde{a})$ so that $[\widetilde{a}]=a \in$ Tor $H_{1}\left(M_{3}, \mathbb{Z}\right) / \mathbb{Z}_{2}$. This is similar to the counting of BPS states in [15]. Note, however, that BPS particles that arise from M2-branes ending on a non-torsion 1-cycles of $M_{3}$ have mass and do not enter into the IR BPS spectrum. Therefore, it is elements in Tor $H_{1}\left(M_{3}, \mathbb{Z}\right) / \mathbb{Z}_{2}$ that give rise to physical boundary conditions which specifies a superselection sector labeled by this brane charge, resulting in a physical BPS Hilbert space $\mathcal{H}_{a}$ with integrality property. In contrast, a flat connection, given by an element of (Tor $\left.H_{1}\left(M_{3}, \mathbb{Z}\right)\right)^{*} / \mathbb{Z}_{2}$ doesn't correspond to any physical boundary conditions. Instead, it is a linear combination of physical boundary conditions leading to a mixture of different charge sectors.

From this point of view, the S-transform in Conjecture 2.1 carries out the change of basis between charges (valued in Tor $H_{1}\left(M_{3}, \mathbb{Z}\right) / \mathbb{Z}_{2}$ ) and holonomies (valued in $\left(\text { Tor } H_{1}\left(M_{3}, \mathbb{Z}\right)\right)^{*} / \mathbb{Z}_{2}$ ) using the natural pairing between them via the "Aharonov-Bohm phase." More precisely, the M2-branes ending on M5-branes produce particles in the effective $3 \mathrm{~d}$ theory carrying electricmagnetic charge $b \in$ Tor $H_{1}\left(M_{3}, \mathbb{Z}\right) / \mathbb{Z}_{2} \cdot{ }^{10}$ On the other hand, $a \in\left(\operatorname{Tor} H_{1}\left(M_{3}, \mathbb{Z}\right)\right)^{*} / \mathbb{Z}_{2}$ specified the holomony, and, by viewing (Tor $\left.H_{1}\left(M_{3}, \mathbb{Z}\right)\right)^{*}$ as the group of characters of Tor $H_{1}\left(M_{3}, \mathbb{Z}\right)$, we have

$$
S_{a b} \propto \sum_{\mathbb{Z}_{2} \text { orbit of } a} \chi_{a}(b)=\sum_{\mathbb{Z}_{2} \text { orbit of } a} e^{4 i \pi \ell k(a, b)} .
$$

Geometrically, $S_{a b}$ is the trace of the holonomy of the flat connection labeled by $a$ along the 1-cycle representing homology class $b$.

Alternatively, one can understand the boundary conditions in the type IIB duality frame of the brane system (2.13), where S-transform can be interpreted as the S-duality of type

\footnotetext{
${ }^{9}$ Not to be confused with the extra "HOMFLY grading" e.g. on the right-hand side of (2.56).

${ }^{10}$ Note that in the case of $N$ M5-branes wrapping $M_{3}$, the M2-branes produce states charged under the magnetic $U(1)^{N} / S_{N}$ (not necessarily $\left.U(N)\right)$ symmetry, as explained in [15].
} 
IIB string theory. Indeed, a quotient of the eleven-dimensional space-time by a circle action $U(1)_{q}$ lands us in type IIA string theory, which can be further T-dualized along the "time" circle $S^{1}$. (Equivalently, these two dualities can be combined into one step, which is the standard M-theory / type IIB duality.) The resulting system involves D3-branes ending on a D5-brane, and S-duality of type IIB string theory maps it into a stack of D3-branes ending on an NS5-brane [18]. Note, that the natural choice of boundary conditions at infinity for a system of D3-branes ending on an NS5-brane is an arbitrary (not necessarily abelian) $S L(2, \mathbb{C})$ flat connection on $M_{3}$. Such choice of a flat connection corresponds to considering analytically continued $S U(2)$ Chern-Simons theory on the Lefschetz thimble associated to that flat connection. However, in general, the corresponding partition function is not continuous in a disk $|q|<1$ due to Stokes phenomena. Instead, in (2.9) we consider quantities $Z_{a}(q)$ which are labeled by abelian flat connections only, and analytic inside $|q|<1$. As explained in [22], for a given value of $\arg k$ one can express $Z_{a}(q)$ as a linear combination of the Feynman path integral on Lefschetz thimbles. If one were to write the S-transform in the basis corresponding to Lefschetz thimbles, instead of $Z_{a}(q)$, the S-matrix would be $k$-dependent.

By definition, each homological block $\widehat{Z}_{a}(q)$ is the graded Euler characteristics (2.24) of $\mathcal{H}_{a}$ that can be computed as an supersymmetric partition function of $T\left[M_{3}\right]$ on $D^{2} \times_{q} S^{1}$ with an $\mathcal{N}=(0,2)$ supersymmetric boundary condition $a$ and metric corresponding to rotation of the disk $D^{2}$ by $\arg q$ when we go around the $S^{1}$,

$$
\widehat{Z}_{a}(q)=Z_{T\left[M_{3}\right]}\left(D^{2} \times_{q} S^{1} ; a\right) .
$$

If one knows the Lagrangian description of $T\left[M_{3}\right]$, this partition function can be computed using localization, see e.g. [29].

Note, if the Lagrangian description of $T\left[M_{3}\right]$ contains chiral multiplets charged under the gauge symmetry, carrying zero R-charge (at the unitarity bound) and with Neumann boundary conditions, then the integral computing the $D^{2} \times{ }_{q} S^{1}$ partition function (i.e. the halfindex) will be singular in general. For example, for $g$ adjoint chiral multiplets the partition function has the following form:

$$
\frac{1}{2} \int_{|z|=1} \frac{d z}{2 \pi i z} \frac{f(z, q)}{\left(1-z^{2}\right)^{g-1}\left(1-z^{-2}\right)^{g-1}}, \quad f(z, q)=f\left(z^{-1}, q\right) \in \mathbb{Z}\left[z, z^{-1}\right][[q]] .
$$

A natural way to regularize it is to take the principle value prescription:

$$
\begin{aligned}
& \frac{1}{2} \text { v.p. } \int_{|z|=1} \frac{d z}{2 \pi i z} \frac{f(z, q)}{\left(1-z^{2}\right)^{g-1}\left(1-z^{-2}\right)^{g-1}} \equiv \\
& \frac{1}{4}\left(\int_{|z|=1+\epsilon}+\int_{|z|=1-\epsilon}\right) \frac{d z}{2 \pi i z} \frac{f(z, q)}{\left(1-z^{2}\right)^{g-1}\left(1-z^{-2}\right)^{g-1}} .
\end{aligned}
$$

As we will see in many examples, this regularization prescription is in agreement with the relation between $\widehat{Z}_{a}(q)=Z_{T\left[M_{3}\right]}\left(D^{2} \times_{q} S^{1} ; a\right)$ and the WRT invariant. This is also the source 
of the $2^{-c}$ factor in (2.7). ${ }^{11}$ The presence of $1 / 2$ factors in $\widehat{Z}_{a}$ can be interpreted as presence of factors $\cong \mathbb{C}[x]$ with $\operatorname{deg}_{q} x=0$ in $\mathcal{H}_{a}$. The $q$-graded Euler charecteristic of $\mathbb{C}[x]$ is naively divergent: $1+1+1+\ldots$, but its zeta-regularization gives $1 / 2$.

\subsection{Superconformal index of $T\left[M_{3}\right]$ and its factorization}

Although the new invariants $\widehat{Z}_{a}(q)$ and their categorification $\mathcal{H}_{a}\left[M_{3}\right]$ have a direct connection to the WRT invariant via (2.6), from the viewpoint of $3 \mathrm{~d} \mathcal{N}=2$ theory $T\left[M_{3} ; S U(2)\right]$, it is not the most natural or simplest object to consider. The main reason is that, in principle, there are infinitely many possible boundary conditions that could be considered. ${ }^{12}$ Identifying the correct subset that appears in (2.16) may be subtle, yet possible, as we shall see in concrete examples.

The more natural object is the superconformal index of $T\left[M_{3}\right]$ or, equivalently, the partition function on $S^{2} \times S^{1}$ with a certain supersymmetry preserving background:

$$
\mathcal{I}(q) \equiv \operatorname{Tr}_{\mathcal{H}_{S^{2}}}(-1)^{F} q^{R / 2+J_{3}}=Z_{T\left[M_{3}\right]}\left(S^{2} \times{ }_{q} S^{1}\right)
$$

where $\mathcal{H}_{S^{2}}$ is the space of BPS states of $3 \mathrm{~d} \mathcal{N}=2 \operatorname{SCFT} T\left[M_{3}\right]$ or, equivalently, $Q$-cohomology of all physical local operators, $F$ is the fermion number, $R$ is the generator of the $U(1)_{R}$ Rsymmetry and $J_{3}$ is the Cartan generator of the $S O(3)$ isometry of $S^{2}$. By construction, this index has the desired integrality property ${ }^{13}$ and can be categorified,

$$
\mathcal{H}_{S^{2}}=\bigoplus_{i, j} \mathcal{H}_{S^{2}}^{i, j}, \quad \mathcal{I}(q)=\sum_{i, j \in \mathbb{Z}} q^{i}(-1)^{j} \operatorname{dim} \mathcal{H}_{S^{2}}^{i, j} \in \mathbb{Z}[[q]]
$$

Another advantage of $\mathcal{H}_{S^{2}}$ compared to $\mathcal{H}_{D^{2}}$ is that it has a natural ring structure which is given by multiplication of BPS operators. One of the statements of $3 \mathrm{~d} / 3 \mathrm{~d}$ correspondence is that the partition function $Z_{T\left[M_{3}\right]}\left(S^{2} \times_{q} S^{1}\right)$ computes the partition function of complex Chern-Simons on $M_{3}$ with real part of the "level" being 0 and analytically continued imaginary part $\propto \tau \equiv \log q /(2 \pi i)$. Motivated by the topological/anti-topological fusion [23] and its recent $3 \mathrm{~d}$ incarnation $[24,25,30,31]$, we would like to make the following conjecture:

\footnotetext{
${ }^{11}$ Such factor can appear even without singularity, when there is only $g=1$ adjoint chiral with R-charge 0 . It cancels the contribution of the vector multiplet, leaving

$$
\frac{1}{2} \int \frac{d z}{2 \pi i z} f(z, q)=\frac{1}{2}\left[z^{0} \text { coefficient of } f(z, q)\right]
$$

where one can take $f(z, q)=1$, as an example.

${ }^{12}$ If we understand the boundary condition as a coupling of the $3 \mathrm{~d}$ theory to a $2 \mathrm{~d}$ theory living on the boundary, then the infinite number of possibilities can be seen, for example, from the fact that we can always introduce a $2 \mathrm{~d}$ theory decoupled from the bulk.

${ }^{13}$ For reasons similar to the ones described at the end of the previous section, in general a negative power of 2 can appear as an overall factor. We omit it in some generic formulas to avoid clutter and instead focus attention on the conceptual structure. As mentioned earlier and as we shall see in examples, the effect of such fractions on categorification can typically be traced to the existence of bosonic modes with zero $q$-grading.
} 
Conjecture 2.2 For $\widehat{Z}_{a}(q)$ as in Conjecture 2.1, the following holds

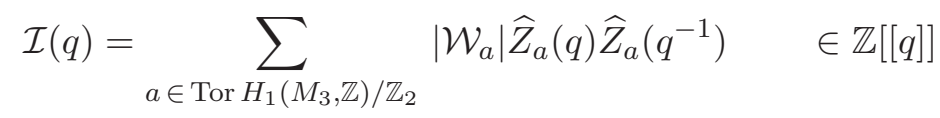

where $\widehat{Z}_{a}(1 / q)$ is an apropriate extension ${ }^{14}$ of $\widehat{Z}_{a}(q)$ to the region $|q|>1$.

\subsection{Further refinement}

As we discussed above, for Seifert 3-manifolds the theory $T\left[M_{3}\right]$ has an extra $U(1)_{\beta}$ flavor symmetry. In particular, this is the case for $M_{3}=L(p, 1)$, which will serve as an important example to us later. This flavor symmetry in $T\left[M_{3}\right]$ results in the presence of an extra $\mathbb{Z}$ grading in homological invariants $\mathcal{H}_{D^{2}}$ and $\mathcal{H}_{S^{2}}$ considered in the previous sections and a possibility to consider the corresponding refined indices (equivariant Euler characteristics):

$$
\widehat{Z}_{a}(q ; t)=2^{-c} \sum_{\substack{i \in \mathbb{Z}+\Delta_{a}, j, \ell \in \mathbb{Z}}} q^{i} t^{\ell}(-1)^{j} \operatorname{dim} \mathcal{H}_{a}^{i, j ; \ell},
$$

and

$$
\mathcal{I}(q ; t)=\sum_{i, j, \ell \in \mathbb{Z}} q^{i} t^{\ell}(-1)^{j} \operatorname{dim} \mathcal{H}_{S^{2}}^{i, j ; \ell}
$$

where, from the physics point of view, $\ell$ is the $U(1)_{\beta}$ charge. The refined indices obviously provide more information about the underlying vector spaces and, as we will see in examples, can be used sometimes to compute (conjecturally) the full homological invariants via the "homological-flavor locking" phenomenon that we will explain later.

The refined version of the factorization formula (2.22) reads

$$
\mathcal{I}(q ; t)=\sum_{a \in \operatorname{Tor}}\left|\mathcal{W}_{a}\right| \widehat{Z}_{a}(q ; t) \widehat{Z}_{a}\left(q^{-1} ; t^{-1}\right) / \mathbb{Z}_{2}
$$

\subsection{Topologically twisted index of $T\left[M_{3}\right]$}

Another interesting invariant of $M_{3}$ which can be realized as an observable of $T\left[M_{3}\right]$ and has a categorification by construction is the topologically twisted index on $S^{2} \times S^{1}$ [32]. Namely, one can consider the $3 \mathrm{~d} \mathcal{N}=2$ theory $T\left[M_{3}\right]$ with a background value of the $U(1)_{R}$-symmetry connection equal to the spin connection on $S^{2}$. In terms of the effective $2 \mathrm{~d} \mathcal{N}=(2,2)$ theory obtained by compactifying $T\left[M_{3}\right]$ on $S^{1}$, this is the familiar A-twist along the $S^{2}$.

\footnotetext{
${ }^{14}$ For a generic 3 -manifold $M_{3}$, the analytic continuation of the series $\widehat{Z}_{a}(q) \in \mathbb{Z}[[q]]$ convergent in $|q|<1$ may not exist outside $|q|=1$, at least in the standard way. However, one possible way to define it for general $M_{3}$ is

$$
\left.\widehat{Z}_{a}\left(q^{-1}\right) \equiv \widehat{Z}_{a}(q)\right|_{M_{3} \rightarrow \bar{M}_{3}},
$$

where $\bar{M}_{3}$ denotes $M_{3}$ with the reversed orientation. Note that therefore $\mathcal{I}(q)$, unlike $\widehat{Z}_{a}(q)$, is insensetive to the orientation.
} 
The topologically twisted index and the underlying homological invariant of $M_{3}$ have the same fugacities/gradings as the superconformal index:

$$
\mathcal{I}_{\text {top }}(q ; t)=\sum_{i, j, \ell \in \mathbb{Z}} q^{i} t^{\ell}(-1)^{j} \operatorname{dim} \mathcal{H}_{S_{\text {top }}^{2}}^{i, j \ell}
$$

As in the superconformal index $\mathcal{I}(q ; t)$, here the parameter $q$ plays the role of the Omegabackground parameter corresponding to rotating $S^{2}$ along one of the axes. However, in general the topologically twisted index $\mathcal{I}_{\text {top }}(q ; t)$ has a much simpler structure (as will be explained later) compared to the superconformal index $\mathcal{I}(q)$. Namely, $\mathcal{I}_{\text {top }}(q ; t)$ is a rational function of $q, t$ (i.e. it has a form of the index of quantum mechanics with two supercharges), whereas $\mathcal{I}(q, t)$ can be as transcendental as, say, a quantum dilogarithm or (Jacobi) mock modular form. Nevertheless, the topologically twisted index is expected to have a similar factorization into homological blocks:

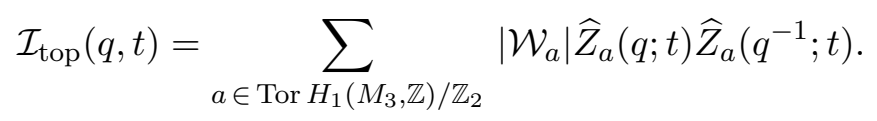

The difference from (2.26) is due to the fact that the supersymmetric backround chosen for superconformal $S^{2} \times S^{1}$ index can be interpreted as doing topological A-twist along one of the $D^{2} \times S^{1}$ halves and anti-A-twist along the other $D^{2} \times S^{1}$ half,

$$
\mathcal{I}(q ; t)=\overline{\mathrm{A}}-\mathrm{twist}
$$

whereas the background for the topologically twisted $S^{2} \times S^{1}$ index is such that the same A-twist is performed along both halves:

$$
\mathcal{I}_{\text {top }}(q ; t)=\mathrm{A}-\text { twist }
$$

\subsection{Generalization to $U(N)$}

For simplicity, in this paper we mostly consider Chern-Simons theory on $M_{3}$ with gauge group being $G=S U(2)$ and the $3 \mathrm{~d} / 3 \mathrm{~d}$ dual theory $T\left[M_{3} ; S U(2)\right]$. However, in principle, everything we say above and below for $S U(2)$ can be easily generalized to the $S U(N)$ or $U(N)$ case; there are no obstructions for that. In particular, the formulae appearing in Conjecture 2.1 generalize as follows for $G=U(N)$ :

$$
\begin{gathered}
Z_{U(N)_{k}}\left[M_{3}\right]=(i \sqrt{2 k})^{N\left(b_{1}\left(M_{3}\right)-1\right) / 2} \sum_{a \in\left(\operatorname{Tor} H_{1}\left(M_{3}, \mathbb{Z}\right)\right)^{N} / S_{N}} e^{\pi i k \sum_{i=1}^{N} \ell k\left(a_{i}, a_{i}\right)} Z_{a}(q), \\
Z_{a}(q)=\left.\sum_{b \in\left(\operatorname{Tor} H_{1}\left(M_{3}, \mathbb{Z}\right)\right)^{N} / S_{N}} e^{\pi i k \sum_{i=1}^{N} \ell k\left(a_{i}, a_{i}\right)} S_{a b} \widehat{Z}_{b}(q)\right|_{q \rightarrow e^{\frac{2 \pi i}{k}}}
\end{gathered}
$$




$$
\begin{aligned}
S_{a b}= & \frac{\sum_{\sigma \in S_{N}} e^{2 \pi i \sum_{i=1}^{N} \ell k\left(a_{i}, b_{\sigma(i)}\right)}}{\left|\operatorname{Stab}_{S_{N}}(a)\right| \cdot\left|\operatorname{Tor} H_{1}\left(M_{3}, \mathbb{Z}\right)\right|^{N / 2}}, \\
& \widehat{Z}_{a}(q) \in(N !)^{-c} q^{\Delta_{a}} \mathbb{Z}[[q]] .
\end{aligned}
$$

As before, using the linking pairing one can identify (Tor $\left.H_{1}\left(M_{3}\right)\right)^{N} / S_{N}$ with the set of connected components of abelian flat connections:

$$
\left(\operatorname{Tor} H_{1}\left(M_{3}\right)\right)^{N} / S_{N} \stackrel{\ell k}{\cong} \operatorname{Hom}\left(\operatorname{Tor} H_{1}\left(M_{3}\right), U(1)^{N}\right) / S_{N} \cong \pi_{0} \mathcal{M}_{\text {flat }}^{\text {ab }}\left(M_{3}, U(N)\right)
$$

where $S_{N}$ is the permutation group of a set with $N$ elements. The factorization formulae (2.26) and (2.28) generalize as follows

$$
\begin{aligned}
& \mathcal{I}(q, t)=\sum_{a \in\left(\operatorname{Tor} H_{1}\left(M_{3}, \mathbb{Z}\right)\right)^{N} / S_{N}}\left|\mathcal{W}_{a}\right| \widehat{Z}_{a}(q, t) \widehat{Z}_{a}\left(q^{-1}, t^{-1}\right), \\
& \mathcal{I}_{\text {top }}(q, t)=\sum_{a \in\left(\operatorname{Tor} H_{1}\left(M_{3}, \mathbb{Z}\right)\right)^{N} / S_{N}}\left|\mathcal{W}_{a}\right| \widehat{Z}_{a}(q, t) \widehat{Z}_{a}\left(q^{-1}, t\right),
\end{aligned}
$$

with

$$
\mathcal{W}_{a} \equiv \operatorname{Stab}_{S_{N}}(a)
$$

Physically, there are several ways to understand the special role of abelian flat connections or, more generally, reducible flat connections for $G$ of higher rank. As we already mentioned in section 2.2, from the viewpoint of quantum field theory (on fivebrane world-volume or its various reductions and limits) this follows directly from the resurgent analysis. On the other hand, by looking at the same system (2.13) from the vantage point of the Calabi-Yau 3-fold, the set (2.35) which labels the new invariants $\widehat{Z}_{a}(q)$ and their categorification $\mathcal{H}_{a}\left[M_{3}\right]$ can be understood as charges of the BPS states or enumerative invariants of the Calabi-Yau 3-fold. We elaborate on this perspective in the following two subsections, expanding the web of dualities and interpretations.

\subsection{Relation to open GW invariants on $T^{*} M_{3}$}

Consider $U(N)$ level- $k$ Chern-Simon theory on a rational homology sphere $M_{3}$,

$$
b_{1}\left(M_{3}\right)=0,
$$

i.e. $H_{1}\left(M_{3}, \mathbb{Z}\right)=$ Tor $H_{1}\left(M_{3}, \mathbb{Z}\right)$ is a finite abelian group. (What follows can be considered as a generalization from the case $H_{1}\left(M_{3}, \mathbb{Z}\right) \cong \mathbb{Z}$ considered in [33]).

Similar to the $S U(2)$ case, $\sum_{i=1}^{N} \ell k\left(a_{i}, a_{i}\right)=\mathrm{CS}(a)$ is the Chern-Simons invariant of the abelian flat connection $a$, and $Z_{a}(q)$ is a Borel resummation [22] of the asymptotic expansion around $a$. Correspondingly, $\widehat{Z}_{b}(q)$ is a Borel resummation of the series of the following type:

$$
\widehat{Z}_{b}(q)=\sum_{m \geq 0} n_{m}^{b} q^{\Delta_{b}+m} \stackrel{\text { resum }}{=} \widehat{Z}_{b}^{\text {pert }}(k)=\sum_{m \geq 1} N_{m}^{b}\left(\frac{2 \pi i}{k}\right)^{m} \in \mathbb{Q}[[2 \pi i / k]] .
$$


Consider

$$
e^{2 \pi i a} \in \operatorname{Hom}\left(H_{1}\left(M_{3}\right), U(1)^{N}\right) / S_{N}
$$

as a formal variable in a generating series. Then $S_{a b}$, for each $b$, up to a factor, can be viewed as a basis element of the symmetric part of the group $\operatorname{ring}^{15}$ of $\left(H_{1}\left(M_{3}, \mathbb{Z}\right)\right)^{N}$ :

$$
\left|H_{1}\left(M_{3}\right)\right|^{N / 2}\left|\operatorname{Stab}_{S_{N}}(b)\right|^{-1} S_{a b} \in \mathbb{Z}\left[\left(H_{1}\left(M_{3}, \mathbb{Z}\right)\right)^{N}\right]^{S_{N}}, \quad b \in\left(H_{1}\left(M_{3}\right)\right)^{N} / S_{N},
$$

if we identify

$$
\prod_{i=1}^{N} e^{2 \pi i \ell k\left(a_{i}, c_{i}\right)} \equiv 1 \cdot c
$$

in the group ring of $\left(H_{1}\left(M_{3}\right)\right)^{N}$.

Then

$$
\left(2 k\left|H_{1}\left(M_{3}\right)\right|\right)^{N / 2}\left|\operatorname{Stab}_{S_{N}}(a)\right| Z_{a}(q) \in \mathbb{Z}\left[\left[q^{1 /\left|H_{1}\right|}\right]\right] \otimes_{\mathbb{Z}} \mathbb{Z}\left[\left(H_{1}\left(M_{3}, \mathbb{Z}\right)\right)^{N}\right]^{S_{N}}
$$

while

$$
\sum_{b \in\left(H_{1}\left(M_{3}\right)\right)^{N} / S_{N}} S_{a b} \widehat{Z}_{b}^{\text {pert }}(k) \in \mathbb{Q}[[2 \pi i / k]] \otimes_{\mathbb{Z}} \mathbb{Z}\left[\left(H_{1}\left(M_{3}, \mathbb{Z}\right)\right)^{N}\right]^{S_{N}} .
$$

On the other hand, one can consider open topological strings (A-model) on $T^{*} M_{3}$ with a Lagrangian brane along $M_{3}$ equipped with a rank- $N$ bundle and an abelian flat connection (local system) a. The free energy is given by

$$
F_{a}^{\text {top }}\left[T^{*} M_{3}\right]\left(g_{s}\right)=\sum_{g, h} g_{s}^{2 g+h-2} \sum_{c \in\left(H_{1}\left(M_{3}, \mathbb{Z}\right)\right)^{h}} N_{g ; c_{1}, \ldots, c_{h}} \prod_{j=1}^{h} e^{2 \pi i \sum_{i=1}^{N} \ell k\left(a_{i}, c_{j}\right)}
$$

where the open GW invariants

$$
N_{g ; c_{1}, \ldots, c_{h}} \in \mathbb{Q}
$$

count pseudoholomorphic maps from a genus- $g$ Riemann surface with $h$ holes to $T^{*} M_{3}$ such that the image of the $j$-th boundary component lands in a homology class $c_{j} \in H_{1}\left(M_{3}, \mathbb{Z}\right)$. The factor

$$
e^{2 \pi i \sum_{i=1}^{N} \ell k\left(a_{i}, c_{j}\right)}=\operatorname{Hol}_{a}\left(c_{j}\right)
$$

\footnotetext{
${ }^{15}$ For example, when $N=2$ and $H_{1}\left(M_{3}, \mathbb{Z}\right)=\mathbb{Z}_{2}$ we have:

$$
\mathbb{Z}\left[\left(H_{1}\left(M_{3}, \mathbb{Z}\right)\right)^{N}\right]^{S_{N}} \cong \mathbb{Z}\left[x_{1}, x_{2}\right]^{S_{2}} /\left\{x_{1}^{2}=1, x_{2}^{2}=1\right\}
$$

and

$$
\begin{aligned}
& S_{a,(0,0)} \propto 1 \\
& S_{a,(1,0)} \propto x_{1}+x_{2} \\
& S_{a,(1,1)} \propto x_{1} x_{2}
\end{aligned}
$$

where $x_{i}=e^{\pi i a}$.
} 
is the holonomy of the abelian flat connection $a$ along $c_{j}$. Note that

$$
N_{g ; c_{1}, \ldots, c_{h}}=0 \quad \text { unless } \quad c_{1}+\ldots+c_{h}=0 \in H_{1}\left(M_{3}, \mathbb{Z}\right)
$$

because there are no non-trivial 2-cycles in $T^{*} M_{3}$. When the flat connection $a$ is trivial, the generating function (2.48) takes a familiar form

$$
F_{0}^{\text {top }}\left[T^{*} M_{3}\right]\left(g_{s}\right)=\sum_{g, h} g_{s}^{2 g+h-2} N_{g ; h} N^{h}
$$

where the averaged version of open GW invariants

$$
N_{g ; h}=\sum_{c \in\left(H_{1}\left(M_{3}, \mathbb{Z}\right)\right)^{h}} N_{g ; c_{1}, \ldots, c_{h}}
$$

"forgets" where the boundary components map to.

Again, using the identification (2.45), the generating function (2.48) can be regarded as

$$
F_{a}^{\text {top }}\left[T^{*} M_{3}\right]\left(g_{s}\right) \in \mathbb{Q}\left[\left[g_{s}\right]\right] \otimes_{\mathbb{Z}} \mathbb{Z}\left[\left(H_{1}\left(M_{3}, \mathbb{Z}\right)\right)^{N}\right]^{S_{N}} .
$$

Then, the relation between CS and open topological strings can be formulated as follows: ${ }^{16}$

$$
\left.\exp F_{a}^{\mathrm{top}}\left[T^{*} M_{3}\right]\left(g_{s}\right)\right|_{g_{s}=\frac{2 \pi i}{k}} \propto \sum_{b} S_{a b} \widehat{Z}_{b}^{\text {pert }}(k)
$$

where the exponent is taken using multiplication rules in the ring in (2.54), the same as the ring ${ }^{17}$ in (2.47). The integer numbers $n_{m}^{b}$ in (2.40) are the "massless variants" of open DT/Ooguri-Vafa invariants of $T^{*} M_{3}$.

\subsection{3-manifolds and the "bottom row"}

Using a relation between the physical realizations of 3-manifold homology and a HOMFLY-PT

\begin{tabular}{|c|c|c|c|}
\hline dor & & & triply-g \\
\hline space-time: & $\mathbb{R} \times T^{*} S^{3} \times T N$ & phase & $T N$ \\
\hline $\begin{array}{r}N \text { M5-branes: } \\
\text { M5'-branes: }\end{array}$ & $\begin{array}{l}\mathbb{R} \times S^{3} \times D^{2} \\
\mathbb{R} \times L_{K} \times D^{2}\end{array}$ & transition & $\begin{aligned} & \mathbb{R} \times X \times T N \\
\mathrm{M}^{\prime}: & \mathbb{R} \times L_{K} \times D^{2}\end{aligned}$ \\
\hline
\end{tabular}
homology, respectively, we can produce a purely mathematical relation between the familiar knot homologies and less familiar 3-manifold homologies of this paper.

Namely, the familiar setup for homology of a knot $K \subset S^{3}$ involves $Q$-cohomology (space of BPS states) of the following system:

\footnotetext{
${ }^{16}$ Normalizations of $Z_{U(N)_{k}}$, as well as the first few terms in $F^{\text {top }}$, are subject to ambiguity, and we won't attempt to fix them here.

${ }^{17}$ Note, that any element of this ring has the form as in the right-hand side of (2.55) since (2.44) are basis elements.
} 
where $L_{K}$ is the conormal bundle of the knot $K$, and $X$ is the resolved conifold, i.e. the total space of $\mathcal{O}(-1) \oplus \mathcal{O}(-1)$ bundle over $\mathbb{C} \mathbf{P}^{1}$.

Note, on the triply-graded ("resolved") side the original M5-branes disappear and we are only left with a system of M5'-branes in a non-trivial Calabi-Yau background $X$. This is very similar to a physical realization of 3-manifold homology in (1.1) or (2.13) where, compared to the right-hand side of (2.56), the Lagrangian 3-manifold $M_{3}$ plays the role of $L_{K}$. Indeed, according to the McLean's theorem, the neighborhood of the Lagrangian submanifold $L_{K} \subset X$ in (2.56) can be identified with the total space of the cotangent bundle,

$$
N\left(L_{K}\right) \cong T^{*} L_{K}
$$

which is precisely the setup of (2.13). There is an important difference, however.

While in (2.13) the Calabi-Yau space is simply the cotangent bundle to the Lagrangian 3-manifold $M_{3}$, in (2.56) it only looks like $T^{*} L_{K}$ in the neighborhood of $L_{K}$. Globally, the topology of $X$ is different from $T^{*} L_{K}$ and, in particular, has non-trivial relative homology group

$$
H_{2}\left(X, L_{K}\right) \cong \mathbb{Z}
$$

It plays an important role in the physical realization of the colored HOMFLY-PT homology of the knot $K$; namely, the conserved charge captured by this relative homology is the so-called $a$-grading of the HOMFLY-PT homology of $K$.

As in [34], we can bridge the gap between the two systems (2.13) and (2.56) by taking the limit $^{18}$

$$
\log (a)=\operatorname{Vol}\left(\mathbb{C} \mathbf{P}^{1}\right) \rightarrow \infty
$$

This limit has a simple interpretation in almost every duality frame. For example, from the vantage point of the Calabi-Yau 3-fold $X$ it corresponds to the limit

$$
X \rightsquigarrow \mathbb{C}^{3}
$$

that, on the toric diagram of the resolved conifold $X$, corresponds to moving two trivalent vertices far away from each other. Keeping only one vertex in sight, we end up with $\mathbb{C}^{3}$ (whose enumerative invariants are counted by the refined topological vertex). After making this replacement in (2.56), we obtain a simpler fivebrane system, whose BPS spectrum $\left(Q_{-}\right.$ cohomology) categorifies only the bottom (resp. top) row of the HOMFLY-PT polynomial that contains the terms with minimal (resp. maximal) a-degree [34].

Now, after taking the limit (2.59)-(2.60), the second cohomology of $X$ is trivial and there is no winding around $\mathbb{C} \mathbf{P}^{1}$ that we had in the original system (2.56). This, of course, corresponds to the fact that, by taking the limit, we lost the $a$-grading focusing only on terms with the lowest $a$-degree. The $q$-degree and $t$-degree, however, are still present and come from $U(1)_{q} \times U(1)_{R}$ symmetry acting on $D^{2} \subset T N$.

\footnotetext{
${ }^{18}$ Note, in relating HOMFLY-PT homology to $\operatorname{sl}(N)$ knot homology one sets $a=q^{N}$, so that $\log (a) \sim N$.
} 


\begin{tabular}{|c|c|}
\hline Knot $K$ & $\mathcal{H}_{\text {bottom }}(K)$ \\
\hline \hline $\mathbf{3}_{\mathbf{1}}$ & $q^{-2}+q^{2} t^{2}$ \\
\hline $\mathbf{4}_{\mathbf{1}}$ & $t^{-2}$ \\
\hline $\mathbf{5}_{\mathbf{1}}$ & $q^{-4}+t^{2}+q^{4} t^{4}$ \\
\hline $\mathbf{5}_{\mathbf{2}}$ & $q^{-2}+t+q^{2} t^{2}$ \\
\hline $\mathbf{6}_{\mathbf{1}}$ & $t^{-2}+q^{-2} t^{-1}$ \\
\hline
\end{tabular}

Table 1: The reduced Poincaré polynomial of the bottom row of the HOMFLY-PT homology for simple knots colored by $\lambda=\square$. The unreduced HOMFLY-PT homology is infinite-dimensional and its Poincaré "polynomial" is, in fact, a power series in $q$ and $t$.

To summarize, by taking the limit (2.59)-(2.60) in the physical system (2.56), we obtain a relation between the bottom row of the HOMFLY-PT homology of $K$ and the homology of the 3-manifold $M_{3}=L_{K}$ :

$$
\mathcal{H}_{\text {bottom }}(K) \cong \mathcal{H}\left(M_{3}\right) .
$$

In fact, the geometry and topology of $M_{3}=L_{K}$ is closely related to that of the knot complement $S^{3} \backslash K$, see [35]. Namely, for a knot (= a link with one component), both $M_{3}=L_{K}$ and $S^{3} \backslash K$ have $H_{1} \cong \mathbb{Z}$. In particular, in both cases the abelian flat connections are labeled by $\mathbb{C}^{*}$-valued holonomies $x_{1}, \ldots, x_{N}$.

Note, (2.61) is a purely mathematical relation whose left-hand side is knot homology and whose right-hand side is a 3-manifold homology. One can explore it and try to make it more concrete, in particular using the physical setup (2.13) and (2.56). First, we need to develop a more precise dictionary between the RHS and LHS of (2.61). Following the above derivation, we see that the M5'-branes in (2.56) correspond to M5-branes in (2.13). Therefore, the $s l(N)$ homology of $M_{3}=L_{K}$ gets related to the bottom row of $\lambda$-colored HOMFLY-PT homology of $K$, where $\lambda$ is a partition with $N$ rows (or $N$ columns). Moreover, as in $[15,33]$, we need to relate the "partition basis" (natural on the LHS of (2.61)) to the "holonomy basis" (natural on the RHS of (2.61)). And, finally, it is important to keep in mind that in relating the physical systems (2.13) and (2.56) we obtain a relation (2.61) that involves unreduced knot homology ${ }^{19}$ in the sense of [13].

As a warm-up, let us take a closer look at the simple case of $N=1$ and $\lambda=\square$. Recall, that $L_{K}$ is a special Lagrangian submanifold in $\mathbb{R}^{6} \cong \mathbb{C}^{3}$. The moduli space $\mathcal{M}\left(L_{K}\right)$ of its Lagrangian deformations, together with a local system that it carries, is the space of SUSY vacua of $3 \mathrm{~d} \mathcal{N}=2$ theory $T\left[L_{K} ; U(1)\right]$ on a circle,

$$
\mathcal{M}\left(L_{K}\right) \cong \mathcal{M}_{\mathrm{SUSY}}\left(T\left[L_{K} ; U(1)\right]\right) \cong \mathcal{M}_{\text {flat }}\left(L_{K}, U(1)_{\mathbb{C}}\right)
$$

\footnotetext{
${ }^{19}$ The reduced HOMFLY-PT homology categorifies normalized HOMFLY-PT polynomial; it is finitedimensional for $\lambda=\square$ and links with one component (i.e. for knots). The unreduced version, on the other hand, categorifies unnormalized HOMFLY-PT polynomial and is infinite-dimensional for any color $\lambda$. The former has no obvious analogue for 3-manifolds.
} 
where in the last relation we used the McLean theorem. The cohomology of this moduli space is precisely the bottom row of the HOMFLY-PT homology of $K$ colored by $\lambda=\square$ [36]:

$$
\mathcal{H}_{\text {bottom }}(K) \cong H^{*}\left(\mathcal{M}\left(L_{K}\right)\right)
$$

In [37], the moduli space $\mathcal{M}\left(L_{K}\right)$ is described as a cluster variety.

Another simple example is $K=$ unknot and $\lambda=S^{r}$ (or $\lambda=\Lambda^{r}$ ), a single-row Young tableaux. The corresponding superpolynomial is

$$
\mathcal{P}_{\text {bottom }}^{S^{r}}(\text { unknot })=\prod_{i=1}^{r} \frac{1}{1-q^{2 i} t^{2(i-1)}} .
$$

These expressions can be found as $x^{r}$-coefficients of $Z_{\text {vortex }}(x ; q, t)$ for a single $3 \mathrm{~d} \mathcal{N}=2$ chiral multiplet. More generally, for $K=$ unknot colored by Young diagrams with $n$ rows (or columns) we end up with the $S^{1} \times D^{2}$ vortex partition function of $n 3 \mathrm{~d} \mathcal{N}=2$ chiral multiplets. This has to be compared with $g l(n)$ homology of $M_{3}=S^{3} \backslash$ (unknot).

It would be useful to develop the relation (2.61) between the familiar HOMFLY-PT homology and the less familiar 3-manifold homology further. We leave this to future work and now turn to detailed analysis of the latter.

\section{Examples}

In this section, we present many examples, illustrating the general proposal outlined in the previous section. In particular, our goal is two-fold: first, we wish to use the proposed physics definition of the new homological invariants $\mathcal{H}_{a}\left[M_{3}\right]$ to compute them in many concrete examples, to the extent that one can start exploring the structure of the results and explicitly test the Conjectures 2.1 and 2.2, which is our second goal. We start with the simplest threemanifold and gradually move to the study of more complex ones.

\section{$3.1 M_{3}=S^{3}$}

For $M_{3}=S^{3}$ and $G=U(N)$, the $3 \mathrm{~d} \mathcal{N}=2$ theory $T\left[S^{3}\right]$ is a $3 \mathrm{~d} \mathcal{N}=2$ Chern-Simons theory with gauge group $U(N)$ at level 1 and an adjoint chiral multiplet $\phi$, whose R-charge is equal to 2 [38]. This theory is dual (the duality is usually referred to as the "duality appetizer" [39-41]) to a system of $N$ free chirals, making it simple to analyze. The R-charges of the free chirals are given by $R=2,4, \ldots, 2 N$, and they have charges $1,2, \ldots, N$ under the $U(1)_{\beta}$ flavor symmetry that rotates the original adjoint chiral $\phi$ by a phase.

As a result, the superconformal index of the theory $T\left[S^{3}\right]$ can be expressed a simple product,

$$
\mathcal{I}_{U(N)}(q, t)=\prod_{i=1}^{N} \frac{\left(t^{-i} q^{1-i} ; q\right)_{\infty}}{\left(t^{i} q^{i} ; q\right)_{\infty}}
$$


where, as usual, the $q$-Pochhammer symbol is defined as

$$
(z ; q)_{n}=\prod_{j=0}^{n-1}\left(1-z q^{j}\right)
$$

Since $H_{1}\left(S^{3}\right)=0$, there is only one homological block $\widehat{Z}_{0}(q, t)$, realized as the $S^{1} \times D^{2}$ partition function of the $N$ free chirals with Neumann boundary conditions,

$$
\widehat{Z}_{0}(q, t)=\prod_{i=1}^{N} \frac{1}{\left(t^{i} q^{i} ; q\right)_{\infty}}
$$

In the terminology of [26] this is an unreduced homological block. ${ }^{20}$ In order to relate it to the WRT invariant of $S^{3}$ as in Conjecture 2.1, before taking the unrefined limit $t \rightarrow 1$, one has to divide by the contribution of the Cartan components of the adjoint chiral $\phi$. The result looks like

$$
\widehat{Z}_{0}(q)=\left.(t q ; q)_{\infty}^{N} \widehat{Z}_{0}(q, t)\right|_{t \rightarrow 1}=\prod_{j=1}^{N-1}\left(1-q^{j}\right)^{N-j}
$$

In the case of $G=S U(2)$, the dual theory consists of just one free chiral multiplet with R-charge 4 and $U(1)_{\beta}$ charge 2 , whose index is

$$
\mathcal{I}_{S U(2)}(q, t)=\frac{\left(t^{-2} q^{-1} ; q\right)_{\infty}}{\left(t^{2} q^{2} ; q\right)_{\infty}} .
$$

and we have

$$
\widehat{Z}_{0}(q, t)=\frac{1}{\left(t^{2} q^{2} ; q\right)_{\infty}}, \quad \widehat{Z}_{0}(q)=\left(1-q^{2}\right)
$$

Using the standard prescription for extending the quantum dilogarithm outside $|q|<1,{ }^{21}$

$$
\left(x ; q^{-1}\right)_{\infty} \propto \frac{1}{(x q ; q)_{\infty}},
$$

we have, for $G=U(N),{ }^{22}$

$$
\widehat{Z}_{0}(1 / q, 1 / t)=\prod_{i=1}^{N} \frac{1}{\left(t^{-i} q^{-i} ; 1 / q\right)_{\infty}}=\frac{1}{N !} \prod_{i=1}^{N}\left(t^{-i} q^{1-i} ; q\right)_{\infty} .
$$

\footnotetext{
${ }^{20}$ Although the terminology "reduced" and "unreduced" here is very similar to the one used in knot homology, there is no direct connection.

${ }^{21}$ Note that this is not an ordinary analytic continuation. The latter actually does not exists because $|q|=1$ is a natural boundary.

${ }^{22}$ Note that there is an ambiguous overall constant in (3.7). We fix it in (3.9) by requiring that the unrefined quantities are related by the ordinary analytic continuation. Namely,

$$
\left.\widehat{Z}_{0}(1 / q, 1 / t) \cdot\left(t^{-1} q^{-1} ; q^{-1}\right)_{\infty}^{N}\right|_{t \rightarrow 1}=\left.\widehat{Z}_{0}(1 / q, 1 / t) \cdot(1 / t ; q)_{\infty}^{-N}\right|_{t \rightarrow 1}=\widehat{Z}_{0}(1 / q)=\prod_{j=1}^{N-1}\left(1-q^{-j}\right)^{N-j}
$$


We see that the superconformal index (3.1) indeed admits a factorization $\grave{a} l a(2.26)$,

$$
\mathcal{I}(q, t)=\left|\mathcal{W}_{0}\right| \widehat{Z}_{0}(q, t) \widehat{Z}_{0}\left(q^{-1}, t^{-1}\right),
$$

with only one homological block in this case.

\subsubsection{Categorifying the index}

As $T\left[S^{3}, U(N)\right]$ is dual to a system of free chirals, its space of BPS states on $S^{2}$ — which gives the homological invariant associated with $M_{3}=S^{3}$ - factorizes as product

$$
\mathcal{H}\left(S^{3}\right)=\mathcal{H}_{2} \otimes \mathcal{H}_{4} \otimes \ldots \otimes \mathcal{H}_{2 N}
$$

where $\mathcal{H}_{2 i}$ is the BPS Hilbert space of a single $3 \mathrm{~d} \mathcal{N}=2$ chiral multiplet with R-charge $2 i$ and $U(1)_{\beta}$ flavor charge $i$. Thus, we now turn to the problem of categorifying the index of a free chiral multiplet.

Recall that for a $3 \mathrm{~d} \mathcal{N}=2$ chiral multiplet $\Phi$ of R-charge $r$ and flavor charge $f$, the superconformal index - equal to the equivariant Euler characteristic of the BPS Hilbert space $\mathcal{H}_{r, f}$ - is given by

$$
\mathcal{I}_{r}(q, t)=\chi_{q, t}\left(\mathcal{H}_{r, f}\right)=\sum_{i, j, \ell \in \mathbb{Z}} q^{i} t^{\ell}(-1)^{j} \operatorname{dim} \mathcal{H}_{r, f}^{i, j ; \ell}=\prod_{j=0}^{\infty} \frac{1-t^{-f} q^{1-r / 2+j}}{1-t^{f} q^{r / 2+j}} .
$$

Each term in this product corresponds to a generator of the BPS Hilbert space $\mathcal{H}_{r, f}$. One can identify the denominator of (3.12) as the contribution of the bosonic modes $\partial^{j} \phi$, and the numerator as the contribution of the fermionic modes $\partial^{j} \bar{\psi}_{+}$. Then $\mathcal{H}_{r, f}$ is freely generated by these generators as a supercommutative algebra,

$$
\mathcal{H}_{r, f}=\mathbb{C}\left[x_{i}, \xi_{i}\right] \cong \Omega^{\bullet}\left(\operatorname{Sym}^{\infty}(\mathbb{C})\right)
$$

with an infinite set of even generators $x_{0}, x_{1}, x_{2}, \ldots$ and odd generators $\xi_{0}, \xi_{1}, \xi_{2}, \ldots$ coming from

$$
\begin{aligned}
& x_{i} \longleftrightarrow \partial^{j} \phi, \\
& \xi_{i} \longleftrightarrow \partial^{j} \bar{\psi}_{+} .
\end{aligned}
$$

In fact, this result has already appeared in math and physics literature (see e.g. [26,34] and references therein), and it is isomorphic to the colored HOMFLY-PT homology of the unknot.

The charges of the generators are summarized below

\begin{tabular}{c|c|c|c|c} 
& $R$ & $F$ & $j_{3}$ & $\frac{R}{2}+j_{3}$ \\
\hline$\phi$ & $r$ & 1 & 0 & $\frac{r}{2}$ \\
\hline $\bar{\psi}_{+}$ & $1-r$ & -1 & $\frac{1}{2}$ & $1-\frac{r}{2}$ \\
\hline$\partial_{+}$ & 0 & 0 & 1 & 1 \\
\hline \hline$\partial^{j} \phi$ & $r$ & 1 & $j$ & $\frac{r}{2}+j$ \\
\hline$\partial^{j} \bar{\psi}_{+}$ & $1-r$ & -1 & $\frac{1}{2}+j$ & $1-\frac{r}{2}+j$
\end{tabular}.


Here $R$ and $F$ are generators of the R-symmetry $U(1)_{R}$ and the flavor symmetry $U(1)_{\beta}$, and $j_{3}$ generates a $U(1)$ subgroup of the $S U(2)$ isometry of the $S^{2}$. Using this data, one can also obtain the Poincaré polynomial of $\mathcal{H}_{r, f}^{i, j ; \ell}$

$$
\mathcal{P}_{r}(q, z, t)=\sum_{i, j, \ell \in \mathbb{Z}} q^{i} z^{j} t^{\ell} \operatorname{dim} \mathcal{H}_{r, f}^{i, j ; \ell}=\prod_{j=0}^{\infty} \frac{1+z^{1-r} t^{-f} q^{1-r / 2+j}}{1-z^{r} t^{f} q^{r / 2+j}}
$$

One interesting observation of [26] is that, for some simple 3 -manifolds $M_{3}$, it is often the case that one can trade the $\ell$-grading for the homological $j$-grading, making the Poincaré polynomial effectively computable. This phenomenon can be seen at the level of $\mathcal{H}_{r, f}$ for a single chiral. Indeed, the $(i, j, \ell)$-degrees of the generators are not independent, and under the substitution of variables $z^{r} \rightarrow t^{f}$ and $z \rightarrow-1$, the Poincaré polynomial for the first two gradings becomes (3.12),

$$
\mathcal{P}_{r}(q, z, 1)=\prod_{j=0}^{\infty} \frac{1+z^{1-r} q^{1-r / 2+j}}{1-z^{r} q^{r / 2+j}} \rightsquigarrow \quad \mathcal{P}_{r}(q,-1, t)=\mathcal{I}_{r}(q, t)=\prod_{j=0}^{\infty} \frac{1-t^{-f} q^{1-r / 2+j}}{1-t^{f} q^{r / 2+j}} .
$$

The homology (3.13) obtained from the index has the same form as the homology on $D^{2} \times{ }_{q} S^{1}$ found in [26]. This could be justified by the following argument. The geometry $S^{1} \times S^{2}$ is conformally flat, and the SUSY variation is obtain from that on flat space. The latter, in turn, is equivalent to partially A-twisted $3 \mathrm{~d} \mathcal{N}=2$ theory, which is precisely the setting of $[26]$.

\subsubsection{The $t=q^{\beta}$ reduction from BPS spectral sequence}

One interesting property of the invariants (3.1) and (3.12) is their behavior under the specialization $t=q^{\beta}$ with $\beta \in \mathbb{Z}$. First, they all become polynomial after taking this limit. For example,

$$
\mathcal{I}_{r, f=1}(q, t) \stackrel{t=q^{\beta}}{\longrightarrow} \prod_{j=0}^{\infty} \frac{1-q^{1-r / 2-\beta+j}}{1-q^{r / 2+\beta+j}}=\left(q^{1-r / 2-\beta} ; q\right)_{r+2 \beta-1},
$$

is simply a polynomial (as opposed to a power series). Second, they exhibit "positivity" in the sense that if one adds a minus sign setting $t=-q^{\beta}$, one finds a polynomial with only positive coefficients:

$$
\mathcal{I}_{r, f=1}\left(q, t=-q^{\beta}\right)=\prod_{j=0}^{r+2 \beta-2}\left(1+q^{1-r / 2-\beta+j}\right) .
$$

Here, these phenomena originate from the fact that, upon setting $t=q^{\beta}$, there is a lot of cancellation between contributions from bosonic and fermionic generators, with only $\bar{\psi}_{+}, \partial \bar{\psi}_{+}, \ldots$, $\partial_{+}^{2 \beta} \bar{\psi}_{+}$remaining. However, as we will see in later part of this paper, these phenomena are actually very universal and also appear in a wide variety of $3 \mathrm{~d} \mathcal{N}=2$ theories $T\left[M_{3}\right]$ with the symmetry $U(1)_{\beta}$. Therefore, it is interesting to understand the $t=q^{\beta}$ reduction at the categorified level. 
The natural uplift of the reduction of superconformal indices

$$
\mathcal{I}_{T\left[M_{3}\right]}(q, t) \quad \rightsquigarrow \quad \mathcal{I}_{T\left[M_{3}\right]}^{(\beta)}(q)=\mathcal{I}\left(q, q^{\beta}\right)
$$

is a BPS spectral sequence [42]:

$$
\mathcal{H}_{Q}^{\mathrm{BPS}} \rightsquigarrow \mathcal{H}_{Q+Q^{\prime}}^{\mathrm{BPS}}
$$

which starts with the space of BPS states of $T\left[M_{3}\right]$ on $S^{2}$ and converges to the space of $Q+Q^{\prime}$-cohomology with a deformed supercharge. For concreteness, we will focus on the example of a single chiral $\Phi=(\phi, \psi)$ with R-charge $r=2$, which can be understood as the theory $T\left[S^{3}, U(1)\right]$. The first step is to explicitly identify the space of BPS states $\mathcal{H}_{S^{2}}$ found in the previous section as the $Q$-cohomology for a supercharge in the following way.

All four supercharges of a $3 \mathrm{~d} \mathcal{N}=2$ theory can be preserved on $S^{2} \times \mathbb{R}$. We pick a supercharge $Q=\bar{Q}_{-}$parametrized by a Killing spinor $\bar{\zeta}_{+}$with

$$
j_{3}\left(\bar{\zeta}_{+}\right)=\frac{1}{2}, \quad R\left(\bar{\zeta}_{+}\right)=-1 .
$$

The supergravity background is given by

$$
V_{\mu}=A_{\mu}^{(R)}=-i \delta_{\mu 3}
$$

with the other components of the background multiplet set to zero. ${ }^{23}$ Then, the on-shell SUSY variation given by $\bar{Q}_{-}$is

$$
\begin{aligned}
\delta \phi & =0 \\
\delta \bar{\psi} & =0 \\
\delta \bar{\phi} & =\sqrt{2} \bar{\zeta}_{+} \bar{\psi}_{-}, \\
\delta \psi & =-\sqrt{2} i \gamma^{\mu} \bar{\zeta}_{+} D_{\mu} \phi+\sqrt{2} i \sigma \phi \bar{\zeta}_{+},
\end{aligned}
$$

where we have included a background $U(1)_{\beta}$ vector multiplet $\left(A_{\mu}, \lambda, \bar{\lambda}, \sigma, D\right)$, and $D_{\mu}$ is a covariant derivative that involves the Levi-Civita connection, $A_{\mu}$ and $A_{\mu}^{(R)}$. Then the $Q-$ closed states are $\phi, \bar{\psi}_{ \pm}$and their derivatives. Among them $\bar{\psi}_{-}, D_{3} \phi$ and $\partial_{-} \phi$ are $Q$-exact, while $D_{3} \bar{\psi}_{+}$and $\partial_{-} \bar{\psi}_{+}$are eliminated by the equation of motion. So we have found that the space of BPS states is exactly the cohomology of $Q=\bar{Q}_{-}$.

One might expect a spectral sequence to arise when $Q$ is deformed into $Q+Q^{\prime}$, whose second page is given by

$$
E_{2}^{\bullet, \bullet}=H^{\bullet}\left(\mathcal{H}_{Q}^{\bullet}, Q^{\prime}\right)
$$

${ }^{23}$ We have chosen the round metric and the veilbein

$$
e_{1}=d \theta, \quad e_{2}=\sin \theta d \varphi, \quad e_{3}=d x_{3}
$$


The simplest scenario is to have only non-trivial differentials on the second page, enabling us to read off $\mathcal{H}_{Q+Q^{\prime}}^{\bullet}$ from the third page, like most examples studied in [42].

When we take $t=q^{\beta}$, we are effectively shifting the $U(1)_{R}$ charge by $2 \beta$ multiple of the $U(1)_{\beta}$ charge. As a result, the operators $\phi$ and $\partial_{+}^{2 \beta+1} \bar{\psi}_{+}$have the same $R / 2+j_{3}$ quantum number and their contribution to the index cancels. Then, naively, we expect to have the following action of $Q^{\prime}$ on $\phi$ :

$$
\left[Q^{\prime}, \phi\right] \sim \partial_{+}^{2 \beta+1} \bar{\psi}_{+}
$$

However, no supercharges can achieve this, even if we turn on more general supergravity backgrounds, as $\phi$ and $\bar{\psi}$ live in different representations of the supersymmetry algebra and don't mix.

Another possibility is to have $\phi$ and $\partial_{+}^{2 \beta+1} \bar{\psi}_{+}$to be eliminated separately. This can be achieved by turning on $-2 \beta+1$ units of $U(1)_{\beta}$ flux along $S^{2}=\mathbb{C} \mathbf{P}^{1} \cdot{ }^{24}$ Now $\phi$ and $\bar{\psi}_{+}$are holomorphic sections of

$$
\phi \in H^{0}\left(\mathbb{C} \mathbf{P}^{1}, O(-2 \beta+1)\right), \quad \bar{\psi}_{+} \in H^{0}\left(\mathbb{C} \mathbf{P}^{1}, O(2 \beta)\right) .
$$

The former has no sections, while the latter has $2 \beta+1$ sections given by $\bar{\psi}_{+}, \partial_{+} \bar{\psi}_{+}, \ldots, \partial_{+}^{2 \beta} \bar{\psi}_{+}$.

At the level of SUSY transformations, we now have a term in $\delta \psi$ of the following form

$$
(\delta \psi)_{-} \sim \gamma^{-} \bar{\zeta}_{+} D_{-} \phi
$$

making all modes of $\phi$ exact under $Q+Q^{\prime}$. On the other hand, the equation of motion for $\bar{\psi}_{+}$will impose

$$
D_{-} \bar{\psi}_{+}=0
$$

but there are solutions to this equation, because $\bar{\psi}$ have negative charge under $U(1)_{\beta}$. It would be interesting to pursue this analysis further in non-trivial examples and also make contact with [43] and [44], where similar phenomena were studied in theories with larger supersymmetry.

\section{$3.2 M_{3}=L(p, 1)$}

\subsubsection{Refined superconformal index}

We now move to the case of $M_{3}=L(p, 1) \cong S^{3} / \mathbb{Z}_{p}$. The theory $T[L(p, 1), U(N)]$ is an $\mathcal{N}=2$ Chern-Simons theory at level $p$, with a chiral multiplet $\Phi$ in the adjoint representation with R-charge $R(\Phi)=2[38,45,46]$. Its superconformal index is given by (see e.g. [47])

$$
\begin{gathered}
\mathcal{I}_{U(N)}(q, t)=\sum_{m_{1} \geqslant \cdots \geqslant m_{N} \in \mathbb{Z}} \frac{1}{\left|\mathcal{W}_{m}\right|} \int_{\left|z_{i}\right|=1} \prod_{j} \frac{d z_{j}}{2 \pi i z_{j}} \prod_{i}^{N}\left(z_{i}\right)^{p m_{i}} \prod_{i \neq j}^{N} t^{-\left|m_{i}-m_{j}\right| / 2} q^{-R\left|m_{i}-m_{j}\right| / 4} \times \\
\left(1-q^{\left|m_{i}-m_{j}\right| / 2} \frac{z_{i}}{z_{j}}\right) \prod_{i \neq j}^{N} \frac{\left(\frac{z_{j}}{z_{i}} t^{-1} q^{\left|m_{i}-m_{j}\right| / 2+1-R / 2} ; q\right)_{\infty}}{\left(\frac{z_{i}}{z_{j}} t q^{\left|m_{i}-m_{j}\right| / 2+R / 2} ; q\right)_{\infty}} \times\left[\frac{\left(t^{-1} q^{1-R / 2} ; q\right)_{\infty}}{\left(t q^{R / 2} ; q\right)_{\infty}}\right]^{N}
\end{gathered}
$$

\footnotetext{
${ }^{24}$ In order for the background to be supersymmetric, one also needs to turn on a constant $\sigma$ for the background gauge multiplet, which won't affect the analysis in this section.
} 
Here $R$ stands for the R-charge of the adjoint chiral multiplet $\Phi$ and the fugacity $t$ is associated to the $U(1)_{\beta}$ flavor symmetry which acts on the adjoint chiral multiplet via $\Phi \mapsto e^{i \theta} \Phi$. Using some computer algebra (e.g. Mathematica) one can calculate explicitly $\mathcal{I}(q, t)$ as a series in $q$ up to a relatively high order. The coefficients are polynomials in $t$, that is

$$
\mathcal{I}(q, t) \in \mathbb{Z}[t][[q]]
$$

For gauge group $G=S U(2)$, the expression for the index simplifies to

$$
\begin{aligned}
\mathcal{I}_{S U(2)}(q, t)=\frac{1}{2} \sum_{m \in \mathbb{Z}} \int \frac{d z}{2 \pi i z} z^{2 p m} t^{-2|m|} q^{-2|m|}\left(1-z^{ \pm 2} q^{|m|}\right) & \\
& \times \frac{(1 / t ; q)_{\infty}\left(z^{ \pm 2} q^{|m|} / t ; q\right)_{\infty}}{(q t ; q)_{\infty}\left(z^{ \pm 2} q^{|m|+1} t ; q\right)_{\infty}}
\end{aligned}
$$

where we use the standard notation

$$
f\left(z^{ \pm 2}\right) \equiv f\left(z^{2}\right) f\left(z^{-2}\right) .
$$

The unreduced homological blocks can be calculated using the following formula [26]:

$$
\begin{aligned}
\widehat{Z}_{a}(q, t)=Z_{T\left[M_{3}\right]}\left(D^{2} \times_{q} S^{1} ; a\right) & = \\
\frac{1}{\left|\mathcal{W}_{a}\right|} & \frac{1}{(t q ; q)_{\infty}^{N}} \int_{\left|z_{i}\right|=1} \prod_{i=1}^{N} \frac{d z_{i}}{2 \pi i z_{i}} \prod_{i \neq j} \frac{\left(z_{i} / z_{j} ; q\right)_{\infty}}{\left(z_{i} / z_{j} t q ; q\right)_{\infty}} \Theta_{a}^{\mathbb{Z}^{N}, p}(z ; q),
\end{aligned}
$$

where $\Theta_{a}^{\mathbb{Z}^{N}} ; p(z ; q)$ is the theta function of the rank- $N$ lattice $\mathbb{Z}^{N}$ with quadratic form $p \cdot \operatorname{Id}$ :

$$
\Theta_{a}^{\mathbb{Z}^{N} ; p}(z, q)=\sum_{n \in p \mathbb{Z}^{N}+a} q^{\sum_{i=1}^{N} n_{i}^{2} / 2 p} \prod_{i=1}^{N} z_{i}^{n_{i}}
$$

We would like to check that the following relation holds:

$$
\mathcal{I}_{U(N)}(q, t)=\sum_{a \in \mathbb{Z}_{p}^{N} / S_{N}}\left|\mathcal{W}_{a}\right| \widehat{Z}_{a}(q, t) \widehat{Z}_{a}(1 / q, 1 / t)
$$

Compared to the case $p=1\left(M_{3}=S^{3}\right)$ considered in section 3.1, there are now multiple homological blocks. Another technical complication is that the formula (3.33) only defines $\widehat{Z}_{a}(q, t)$ for $|q|<1$, since the theta function is only given in terms of series convergent in $|q|<1$, with no canonical analytic continuation outside of the unit disk.

This problem will be resolved in section 3.2.4. For now, let us note that in the unrefined case $(t=1)$ such problem does not appear because

$$
\widehat{Z}_{a}(q)=\left.\frac{\widehat{Z}_{a}(q, t)}{(t q ; q)_{\infty}^{N}}\right|_{t \rightarrow 1}=\frac{1}{\left|\mathcal{W}_{a}\right|} \int_{\left|z_{i}\right|=1} \prod_{i=1}^{N} \frac{d z_{i}}{2 \pi i z_{i}} \prod_{i \neq j}\left(1-z_{i} / z_{j}\right) \Theta_{a}^{\mathbb{Z}^{N}, p}(z ; q) \quad \in \mathbb{Z}[q]
$$


is just a polynomial and is obviously well-defined for any $|q|<\infty$. Note, that the factorization of the superconformal index in the unrefined case was essentially checked in [41]. There it was shown that

$$
\mathcal{I}_{U(N)}(q)=\sum_{a \in \mathbb{Z}_{p}^{N} / S_{N}}\left|\mathcal{W}_{a}\right| Z_{a}(q) Z_{a}(1 / q)
$$

where $^{25}$

$$
\mathcal{I}_{U(N)}(q)=\left.\mathcal{I}_{U(N)}(q, t) \cdot \frac{(t q ; q)_{\infty}^{N}}{(1 / t ; q)_{\infty}^{N}}\right|_{t \rightarrow 1}
$$

and $Z_{a}(q)=\sum_{b} S_{a b} \widehat{Z}_{b}(q)$ is as in (2.31), the contributions of flat connection $a$ to WRT invariant/CS partition function on $M_{3}=L(p, 1)$. Then

$$
\mathcal{I}_{U(N)}(q)=\sum_{a \in \mathbb{Z}_{p}^{N} / S_{N}}\left|\mathcal{W}_{a}\right| \widehat{Z}_{a}(q) \widehat{Z}_{a}(1 / q)
$$

follows from (3.37) and $S^{2}=\operatorname{Id}(2.10)$ along with the symmetry

$$
\left|W_{a}\right| S_{a b}=\left|W_{b}\right| S_{b a}
$$

\subsubsection{Topologically twisted index of $T[L(p, 1)]$}

For group $G=S U(2)$, the topologically twisted index (refined by angular momentum) of $T[L(p, 1)]$ reads $[32]$ :

$$
\mathcal{I}_{\mathrm{top}}(q, t)=\frac{1}{2} \sum_{m \in \mathbb{Z}_{\mathrm{JK}}} \int_{\frac{d z}{2 \pi i z}} \frac{z^{2(p+2) m} q^{-m}\left(1-z^{2} q^{m}\right)\left(1-z^{-2} q^{m}\right)}{\left(z^{2} t q^{1-m} ; q\right)_{2 m-1}\left(z^{-2} t q^{1+m} ; q\right)_{-2 m-1}(t q ; q)_{-1}},
$$

where, as usual, the Pochhammer symbol with negative integer in the subscript is defined via the following identity:

$$
(x ; q)_{n}=\frac{1}{\left(x q^{n} ; q\right)_{-n}} .
$$

The contour in (3.54) is chosen according to the Jeffrey-Kirwan residue prescription. Namely, we either choose poles at $z=0, z= \pm \sqrt{t} q^{\cdots}$ or at $z=\infty, z= \pm 1 / \sqrt{t} q^{\cdots}$. The result is

${ }^{25}$ The full index $\mathcal{I}_{U(N)}(q, t)$ of $T[L(p, 1)]$ vanishes in the unrefined limit $t \rightarrow 1$,

$$
\mathcal{I}_{U(N)}(q, t) \sim\left(1-t^{-1}\right)^{N} \mathcal{I}_{U(N)}(q),
$$

due to the contribution of Cartan components of the adjoint chiral multiplet with R-charge 2, which saturate the unitarity bound. The rescaling in (3.40) removes their contribution and makes the limit finite. Another method of regularization is to take the same limit but in the form $t=q^{\epsilon}, \epsilon \rightarrow 0$, which correponds to taking R-charge to be $2-\epsilon$, and then just remove an appropriate power of $\epsilon$ :

$$
\mathcal{I}_{U(N)}^{\prime}(q)=\lim _{\epsilon \rightarrow 0} \mathcal{I}_{U(N)}(q, t) \cdot \epsilon^{-N}=(\log q)^{N} \mathcal{I}_{U(N)}(q) .
$$

This is the approach that was used in [41]. Then the $(\log q)^{N} \propto k^{-N}$ factor agrees with the factorization if we include the overall factor $k^{-N / 2}$ in (2.31) into the definition of $Z_{a}(q)$. 
strikingly simple:

$$
\mathcal{I}_{\text {top }}(q, t)=\left\{\begin{array}{clrl}
\frac{1}{\left(t^{2} q^{2} ; q\right)_{-3}} & \equiv\left(t^{2} / q ; q\right)_{3}, & p=1, \\
1-t^{4}, & & p=2, \\
1-t^{3}, & & p \geq 3 .
\end{array}\right.
$$

As expected, the result for $p=1$ is in agreement with the dual description by a free chiral multiplet with R-charge 4 . Note that for $p>1$ the result turns out to be $q$-independent. In fact, for large $p$, the twisted index of $T[L(p, 1) ; G]$ with a general Lie group $G$ becomes

$$
\mathcal{I}_{\text {top }} \stackrel{p \gg 1}{\longrightarrow} P_{-t}(G)
$$

where $P_{-t}(G)$ denotes the Poincaré polynomial of $G$ in variable $-t$. For $G=S U(2)$,

$$
P_{-t}(G)=1-t^{3}
$$

and, for $S U(3)$, one has [48]:

$$
\mathcal{I}_{\text {top }} \stackrel{p \gg 1}{\longrightarrow} 1-t^{3}-t^{5}+t^{8}=P_{-t}(S U(3)) .
$$

Additionally, the BPS spectrum can be identified with the cohomology of $G$,

$$
\mathcal{H}_{\mathrm{tw}-\mathrm{BPS}}^{\bullet}=H^{\bullet}(G)
$$

An argument for this relation mentioned above is essentially given in section 5 of [49] after Proposition 3, as summarized in the following.

The twisted index of $T[L(p, 1)]$ computes the equivariant index of certain K-theory class over $\mathfrak{M}_{H}\left(\mathbb{C P}^{1} ; G\right)$, the Hitchin moduli stack over $\mathbb{C P}^{1},{ }^{26}$

$$
\mathcal{I}_{\text {top }}(q, t)=\operatorname{Ind}_{t}\left(\mathfrak{M}_{H}, \mathcal{F}_{q} \otimes L^{p}\right) .
$$

Here, $\mathfrak{M}_{H}$ is a complicated derived stack, but using the projection map $\mathfrak{M}_{H}\left(\mathbb{C} \mathbf{P}^{1}\right) \rightarrow \mathfrak{M}\left(\mathbb{C P}^{1}\right)$ to the moduli stack of $G_{\mathbb{C}^{-} \text {bundles over }} \mathbb{C P}^{1}$, the above index can be written as ${ }^{27}$

$$
\mathcal{I}_{\text {top }}(q, t)=\operatorname{Ind}\left(\mathfrak{M}, S_{t} T_{\mathfrak{M}} \otimes \mathcal{F}_{q} \otimes L^{p}\right),
$$

and can be further decomposed into a summation over different strata of $\mathfrak{M}\left(\mathbb{C} \mathbf{P}^{1}\right)$, given by Grothendieck's classification theorem.

For sufficiently large $p$, the contribution from the unstable strata all vanish. And the semi-stable stratum is the classifying stack $B G_{\mathbb{C}}$ (it could be viewed as a single point -

\footnotetext{
${ }^{26}$ In this equation, $L$ is the determinant line bundle and $\mathcal{F}_{q}$ is an object in the derived category of coherent sheaves on $\mathfrak{M}_{H}$ with dependence on $q$. In general, as explain in [38,48], the twisted partition function of $T[L(k, 1)]$ on $S^{1} \times \Sigma$ gives the "equivariant Verlinde formula," which in turn can be written as a K-theory index over $\mathfrak{M}_{H}(\Sigma)$. This fact, combined with the projection map $\mathfrak{M}_{H} \rightarrow \mathfrak{M}$, is the starting point for the proof of the equivariant Verlinde formula $[49,50]$.

${ }^{27}$ Here $S_{t} T_{\mathfrak{M}}$ denotes the total symmetric power of the tangent complex of $\mathfrak{M}$.
} 
the moduli space - with $G_{\mathbb{C}}$ as the stabilizer). As both $L^{p}$ and $\mathcal{F}_{q}$ are trivial over $B G_{\mathbb{C}}$, both $q$ and $p$ dependence disappears, and the problem only depends on the choice of $G$. In this simple case, one can actually directly compute the cohomology groups (BPS states) that contribute to the index in (3.51)

$$
\mathcal{H}_{\mathrm{tw}-\mathrm{BPS}}^{\bullet}=H^{\bullet}\left(\mathfrak{M}, S_{t} T_{\mathfrak{M}} \otimes \mathcal{F}_{q} \otimes L^{p}\right) .
$$

The tangent space of $B G_{\mathbb{C}}$ is given by the two-step complex $\mathfrak{g} \rightarrow 0$ with $\mathfrak{g}$ placed at degree -1 . So in the end, one has

$$
\mathcal{H}_{\mathrm{tw}-\mathrm{BPS}}^{j}=H_{G}^{-j}\left(\Lambda^{j} \mathfrak{g}[j]\right)=\left(\Lambda^{j} \mathfrak{g}\right)^{G}=H^{j}(G),
$$

with the BPS states represented by the elements in the cohomolgy of the group $G$, or equivalently, elements in $\left(\Lambda^{\bullet} \mathfrak{g}\right)^{G}$. And, in this concrete example, one again finds that $t$-degree agrees with cohomological degree. The "(co-)homological-flavor locking" in this case is a result of the tangent complex $\mathfrak{g} \rightarrow 0$ being concentrated in degree -1 .

In fact, the above explanation using the stack language can be readily "translated" into a physics argument as follows. When $p$ is sufficiently large, in the topologically twisted index of $T\left[M_{3}\right]$ only the zero-flux sector $m=0$ survives - this is what mathematicians would call the "semi-stable stratum." Then, the index essentially becomes the index of the $\mathcal{N}=2 \mathrm{SQM}$ (which can be considered as a reduction of a $2 \mathrm{~d} \mathcal{N}=(0,2)$ theory) with gauge group $G$ and an adjoint Fermi multiplet $\Psi$ of $U(1)_{\beta}$ charge 1. In particular, for $G=S U(2)$ we have

$$
\mathcal{I}_{\text {top }}(q, t)=\frac{1}{2} \sum_{m \in \mathbb{Z}} \int_{\mathrm{JK}} \frac{d z}{2 \pi i z}\left(1-z^{ \pm 2}\right)\left(1-t z^{ \pm 2}\right)(1-t)=\left(1-t^{3}\right) .
$$

In the IR, such theory should be effectively described by gauge-invariant combinations of Fermi-fields, corresponding precisely to generators of $G$-invariant part of the exterior algebra $(\Lambda \mathfrak{g})^{G}$, which, in turn, can be understood as generators of the cohomology of the Lie group. For $G=S U(N)$ these correspond to $\operatorname{Tr} \Psi^{3}, \operatorname{Tr} \Psi^{5}, \ldots, \operatorname{Tr} \Psi^{2 N-1}$ in the SQM.

Although the topological index looks much simpler compared to the superconformal index, it should also admit a factorization into the homological blocks via (2.28). For $G=S U(2)$, we would like to check

$$
\mathcal{I}_{\text {top }}(q, t)=\sum_{a \in \mathbb{Z}_{p} / \mathbb{Z}_{2}}\left|\mathcal{W}_{a}\right| \widehat{Z}_{a}(q, t) \widehat{Z}_{a}(1 / q, t)
$$

It is easy to see how this works for $p=1\left(M_{3}=S^{3}\right)$ :

$$
\widehat{Z}_{0}(q, t) \cdot \widehat{Z}_{0}(1 / q, t)=\frac{1}{\left(t^{2} q^{2} ; q\right)_{\infty}} \cdot \frac{1}{\left(t^{2} q^{-2} ; q^{-1}\right)_{\infty}}=\frac{\left(t^{2} q^{-1} ; q\right)_{\infty}}{\left(t^{2} q^{2} ; q\right)_{\infty}}=\left(t^{2} / q ; q\right)_{3} .
$$

In the case $p>1$, again, the problem of defining $\widehat{Z}_{a}(1 / q, t)$ arises. It will be resolved for both superconformal and topologically twisted index in section 3.2.4. 


\subsection{3 $t=q^{\beta}$ reduction}

Consider the case of $G=S U(2)$. Instead of taking the unrefined limit $t \rightarrow 1$, one can consider more general ${ }^{28}$ limit $t \rightarrow q^{\beta}$, with $\beta \in \mathbb{Z}$. One can show that in such a limit the reduced (that is with removed contribution of the Cartan component of the adjoint chiral) the superconformal index given by formula (3.54) becomes a Laurent polynomial in $q$ or a rational function, depending on the sign of $\beta$ :

$$
\mathcal{I}^{(\beta)}(q) \equiv \lim _{t \rightarrow q^{\beta}} \mathcal{I}(q, t) \frac{(q t ; q)_{\infty}}{(1 / t ; q)_{\infty}} \in\left\{\begin{array}{cc}
\mathbb{Z}\left[q, q^{-1}\right], & \beta \in \mathbb{Z}_{+}, \\
\mathbb{Z}(q), & \beta \in-\mathbb{Z}_{+} .
\end{array}\right.
$$

Similarly, for topologically twisted index we have

$$
\mathcal{I}_{\text {top }}^{(\beta)}(q) \equiv \lim _{t \rightarrow q^{\beta}} \mathcal{I}_{\text {top }}(q, t)(q t ; q)_{-1}=\lim _{t \rightarrow q^{\beta}} \frac{\mathcal{I}_{\text {top }}(q, t)}{1-t},
$$

while for the homological blocks we obtain

$$
\left.\widehat{Z}_{a}^{(\beta)}(q) \equiv(q t ; q)_{\infty} \widehat{Z}_{a}(q, t)\right|_{t=q^{\beta}} \in q^{\frac{a^{2}}{p}} \cdot\left\{\begin{array}{l}
\mathbb{Z}[q], \beta \in \mathbb{Z}_{+} \\
\mathbb{Z}(q), \beta \in-\mathbb{Z}_{+}
\end{array}\right.
$$

Explicitly, we have

$$
\widehat{Z}_{a}^{(\beta)}(q)=\frac{1}{\left|\mathcal{W}_{a}\right|} \int \frac{d z}{2 \pi i z}\left(z^{2} ; q\right)_{\beta+1}\left(z^{-2} ; q\right)_{\beta+1} \sum_{n \in p \mathbb{Z}+a} q^{n^{2} / p} z^{2 n}
$$

Taking into account that

$$
\left(q^{-1} t^{-1} ; q^{-1}\right)_{\infty}=\frac{1}{(1 / t ; q)_{\infty}}
$$

the formula (3.35) then reduces to the following relation between Laurent polynomials (for $\beta \in \mathbb{Z}_{+}$) or rational functions (for $\beta \in-\mathbb{Z}_{+}$):

$$
\mathcal{I}^{(\beta)}(q)=\sum_{a \in \mathbb{Z}_{p} / \mathbb{Z}_{2}}\left|\mathcal{W}_{a}\right| \widehat{Z}_{a}^{(\beta)}(q) \widehat{Z}_{a}^{(\beta)}(1 / q)
$$

For the topologically twisted index we have instead

$$
\mathcal{I}_{\text {top }}^{(\beta)}(q)=\sum_{a \in \mathbb{Z}_{p} / \mathbb{Z}_{2}}\left|\mathcal{W}_{a}\right| \widehat{Z}_{a}^{(\beta)}(q) \widehat{Z}_{a}^{(-\beta)}(1 / q)
$$

These relations are easy to check for various values of $p$ and $\beta$. For example, for $p=5$ we should check

$$
\mathcal{I}^{(\beta)}(q)=2 \widehat{Z}_{0}^{(\beta)}(q) \widehat{Z}_{0}^{(\beta)}(1 / q)+\widehat{Z}_{1}^{(\beta)}(q) \widehat{Z}_{1}^{(\beta)}(1 / q)+\widehat{Z}_{2}^{(\beta)}(q) \widehat{Z}_{2}^{(\beta)}(1 / q)
$$

\footnotetext{
${ }^{28}$ A similar reduction was considered in [19] for refined Chern-Simons theory as a way to circumvent certain technicalities.
} 
and

$$
\mathcal{I}_{\text {top }}^{(\beta)}(q)=\widehat{Z}_{0}^{(\beta)}(q) \cdot 2 \widehat{Z}_{0}^{(-\beta)}(1 / q)+\widehat{Z}_{1}^{(\beta)}(q) \widehat{Z}_{1}^{(-\beta)}(1 / q)+\widehat{Z}_{2}^{(\beta)}(q) \widehat{Z}_{2}^{(-\beta)}(1 / q) .
$$

The particular expressions for a few first values of $\beta$ are as follows.

For $\beta=0$,

$$
\begin{aligned}
& \widehat{Z}_{0}^{(0)}(q)=1, \widehat{Z}_{1}^{(0)}(q)=-q^{1 / 5}, \widehat{Z}_{2}^{(0)}(q)=0 . \\
& \mathcal{I}^{(0)}(0)=3, \\
& \mathcal{I}_{\text {top }}^{(0)}(0)=3 .
\end{aligned}
$$

For $\beta=1,{ }^{29}$

$$
\begin{gathered}
\widehat{Z}_{0}^{(1)}(q)=1+q+q^{2}, \widehat{Z}_{1}^{(1)}(q)=-q^{1 / 5}\left(1+2 q+q^{2}\right), \widehat{Z}_{2}^{(1)}(q)=q^{9 / 5} \\
2 \widehat{Z}_{0}^{(-1)}(q)=1, \widehat{Z}_{1}^{(-1)}(q)=0, \widehat{Z}_{2}^{(-1)}(q)=0 \\
\mathcal{I}^{(1)}(q)=\frac{3}{q^{2}}+\frac{8}{q}+13+8 q+3 q^{2} \\
\mathcal{I}_{\text {top }}^{(1)}(q)=1+q+q^{2} .
\end{gathered}
$$

For $\beta=2$,

$$
\begin{gathered}
\widehat{Z}_{0}^{(2)}(q)=1+q+2 q^{2}+2 q^{3}+2 q^{4}+q^{5}+q^{6}, \\
\widehat{Z}_{1}^{(2)}(q)=-q^{1 / 5}\left(1+2 q+3 q^{2}+3 q^{3}+3 q^{4}+2 q^{5}+q^{6}\right), \\
\widehat{Z}_{2}^{(2)}(q)=q^{9 / 5}\left(1+q+2 q+q^{4}\right) . \\
2 \widehat{Z}_{0}^{(-2)}(q)=-q^{2} /\left(1-q^{2}\right), \\
\widehat{Z}_{1}^{(-2)}(q)=-q^{6 / 5} /\left(1-q^{2}\right), \\
\widehat{Z}_{2}^{(-2)}(q)=-q^{4 / 5} /\left(1-q^{2}\right) . \\
\mathcal{I}^{(2)}(q)=\frac{3}{q^{6}}+\frac{8}{q^{5}}+\frac{21}{q^{4}}+\frac{35}{q^{3}}+\frac{55}{q^{2}}+\frac{65}{q}+76+65 q+55 q^{2}+35 q^{3}+21 q^{4}+8 q^{5}+3 q^{6}, \\
\mathcal{I}_{\text {top }}^{(2)}(q)=1+q^{2}+q^{4} .
\end{gathered}
$$

And for $\beta=3$,

$$
\begin{gathered}
\widehat{Z}_{0}^{(3)}(q)=q^{12}+q^{11}+2 q^{10}+3 q^{9}+4 q^{8}+4 q^{7}+5 q^{6}+4 q^{5}+4 q^{4}+3 q^{3}+2 q^{2}+q+1, \\
\widehat{Z}_{1}^{(3)}(q)=-q^{1 / 5}\left(q^{12}+2 q^{11}+3 q^{10}+4 q^{9}+6 q^{8}+7 q^{7}+8 q^{6}+7 q^{5}+6 q^{4}+5 q^{3}+3 q^{2}+2 q+1\right), \\
\widehat{Z}_{2}^{(3)}(q)=q^{9 / 5}\left(q^{10}+2 q^{8}+2 q^{7}+2 q^{6}+3 q^{5}+3 q^{4}+2 q^{3}+3 q^{2}+q+1\right) . \\
2 \widehat{Z}_{0}^{(-3)}(q)=\left(q^{7}+q^{5}+q^{4}+q^{3}+q^{2}\right) /\left(\left(1-q^{3}\right)\left(1-q^{4}\right)\right), \\
\widehat{Z}_{1}^{(-3)}(q)=q^{11 / 5}\left(q^{4}+q^{3}+q^{2}+q+1\right) /\left(\left(1-q^{3}\right)\left(1-q^{4}\right)\right), \\
\widehat{Z}_{2}^{(-3)}(q)=q^{14 / 5}\left(q^{3}+q^{2}+2 q+1\right) /\left(\left(1-q^{3}\right)\left(1-q^{4}\right)\right) . \\
\mathcal{I}_{\text {top }}^{(3)}(q)=1+q^{3}+q^{6}
\end{gathered}
$$

\footnotetext{
${ }^{29}$ Notice that after removing a $(1-t)$ factor from $\mathcal{I}^{\text {top }}(q, t)$, we are left with $\left(1-t^{3}\right) /(1-t)=1+t+t^{2}$.
} 
Notice that the coefficients of the $q$-expansions are always positive (up to an overall sign), and this reflects that the BPS space $\mathcal{H}_{a}^{(\beta)}$ obtained from the spectral sequence (3.19), now doubly-graded by the eigenvalues under the $U(1)_{q} \times U(1)_{R}$ action,

$$
\mathcal{H}_{a}^{(\beta)}=\bigoplus_{i, j \in \mathbb{Z}} \mathcal{H}_{a}^{(\beta) i, j}
$$

enjoys some highly non-trivial properties — subspaces with odd $j$-degree are either all empty, or have their contributions canceled when we take the equivariant Euler characteristic. Moreover, the spectral sequence (3.19) is consistent with the following property, which one can check explicitly order by order in $q$ :

$$
(-q t ; q)_{\infty} \widehat{Z}_{a}(q,-t)=\widehat{Z}_{a}^{(\beta)}(q)+\left(1+q^{\beta} t^{-1}\right) P_{+}(q, t)
$$

where all the coefficient are positive (up to an overall common sign). Namely,

$$
(-q t ; q)_{\infty} \widehat{Z}_{a}(q,-t), P_{+}(q, t) \in \pm \mathbb{Z}_{+}[t][[q]], \quad \widehat{Z}_{a}(q) \in \pm \mathbb{Z}_{+}[q]
$$

The second term in (3.71) represent the pairs of states that go away in the cohomology w.r.t. the deformed supercharge.

Also, the complexity of the blocks $\widehat{Z}_{a}^{(\beta)}$ and $\widehat{Z}_{a}^{(-\beta)}$ will grow with $\beta$, this is expected from the discussion in section 3.1.2 via spectral sequence, where we have seen more cancellations for smaller $\beta$ and fewer ones for larger $\beta$.

\subsubsection{Cyclotomic expansion}

In this secition we show how to obtain expression for $\widehat{Z}_{a}(1 / q, t)$ with $|q|<1$ using what is usually called the cyclotomic expansion.

Let us first consider the general construction. Let $F(q, t)$ be some function of $q$ and $t$, and suppose we know its restrictions at $t=q^{\beta}$, with $\beta \in \mathbb{Z}$,

$$
F^{(\beta)}(q) \equiv F\left(q, q^{\beta}\right)
$$

Then, one can write the following formal cyclotomic-like expansion for $F(q, t)$ :

$$
F(q, t)=\sum_{m=0}^{\infty} \widetilde{F}^{(m)}(q) \cdot \frac{t^{m}(1 / t ; q)_{m}}{(q ; q)_{m}} .
$$

The coefficient of the cyclotomic expansion, $\widetilde{F}^{(m)}(q)$, are related to $F^{(m)}(q)$ by a "triangular" linear transform:

$$
\begin{aligned}
& \widetilde{F}^{(0)}(q)=F^{(0)}(q) \\
& \widetilde{F}^{(1)}(q)=F^{(0)}(q)-F^{(1)}(q) \\
& \widetilde{F}^{(2)}(q)=F^{(0)}(q)-\left(1+q^{-1}\right) F^{(1)}(q)+q^{-1} F^{(2)}(q) \\
& \widetilde{F}^{(3)}(q)=F^{(0)}(q)-\left(1+q^{-1}+q^{-2}\right) F^{(1)}(q)+\left(q^{-1}+q^{-2}+q^{-3}\right) F^{(2)}(q)-q^{-4} F^{(3)}(q)
\end{aligned}
$$


Note, that the denominators $(q ; q)_{m}$ in $(3.74)$ are introduced for convenience, so that

$$
F^{(\beta)}(q) \in \mathbb{Z}\left[q, q^{-1}\right] \quad \Longleftrightarrow \quad \widetilde{F}^{(m)}(q) \in \mathbb{Z}\left[q, q^{-1}\right]
$$

but in principle they can be absorbed into the definition of cyclotomic coefficients $\widetilde{F}^{(m)}(q)$.

If the minimal degree of $q$ in $\widetilde{F}^{(m)}(q)$ grows with $m$, one can produce a $q$-expansion from the cyclotomic expansion (3.74). However, such procedure is quite formal and in general might not actually give the actual $q$-expansion that converges to $F(q, t)$ when $|q|<1$. But it can be done for homological blocks. In particular, from (3.59) one obtains the following cyclotomic-like expansion:

$$
\widehat{Z}_{a}(q, t)=\frac{1}{(q t ; q)_{\infty}} \sum_{m=0}^{\infty} \widetilde{\widehat{Z}}_{a}^{(m)}(q) \cdot \frac{t^{m}(1 / t ; q)_{m}}{(q ; q)_{m}}
$$

where the coefficients

$$
\widetilde{\widehat{Z}}_{a}^{(m)}(q) \in \mathbb{Z}[q]
$$

turn out to be polynomials in $q$ (non-Laurent) with minimal power growing linearly in $m$. Expanding each term in $q$ then gives us a $q$-series of the following form:

$$
\widehat{Z}_{a}(q, t)=\sum_{n=0}^{\infty} P_{n}(t) q^{n}, \quad P_{n}(t) \in \mathbb{Z}[t],
$$

which coincides with the $q$-series that can be produced directly from (3.33). On the other hand, since $P_{m}(t)$ contain only positive powers of $t$, one can produce $\widehat{Z}^{(\beta)}(q)$ - or, equivalently, the coefficients of the cyclotomic expansion $\widetilde{\widehat{Z}}^{(\beta)}(q)$ - from (3.33) just by making the substitution $t=q^{\beta}$ and observing that the resulting $q$-series truncates.

Therefore, we assume that for anti-blocks one can write similarly:

$$
\widehat{Z}_{a}(1 / q, t)=(1 / t ; q)_{\infty} \sum_{m=0}^{\infty} \widetilde{\widehat{Z}}_{a}^{(-m)}(1 / q) \cdot \frac{t^{m}(1 / t ; q)_{m}}{(q ; q)_{m}}
$$

where $\widetilde{\widehat{Z}}_{a}^{(-m)}(1 / q)$ are related to $\left.\widehat{Z}_{a}^{(-\beta)}(1 / q) \equiv\left(q^{-1} t ; q^{-1}\right)_{\infty} \widehat{Z}_{a}\left(q^{-1}, t\right)\right|_{t=q^{\beta}}$ in the same way as $\widetilde{F}^{(m)}(q)$ are related to $F^{(\beta)}(q)$ in $(3.75)$.

For example, in the case $p=5$, using expressions for $\widehat{Z}_{a}^{(-\beta)}(q)$ calculated in Section 3.2.3 we have

$$
\begin{aligned}
& 2 \widehat{Z}_{0}(1 / q, t)=\left(-t^{3}+t^{2}-t+1\right)+\left(t^{4}-3 t^{3}+5 t^{2}-3 t\right) q+\left(-t^{5}+6 t^{4}-11 t^{3}+11 t^{2}-6 t+1\right) q^{2} \\
& +\left(t^{6}-6 t^{5}+17 t^{4}-28 t^{3}+26 t^{2}-12 t+2\right) q^{3}+O\left(q^{4}\right), \\
& \widehat{Z}_{1}(1 / q, t)=q^{-1 / 5}\left(\left(t^{2}-t\right)+\left(t^{4}-3 t^{3}+3 t^{2}-2 t+1\right) q+\left(-t^{5}+4 t^{4}-9 t^{3}+10 t^{2}-5 t+1\right) q^{2}\right. \\
& \left.+\left(-4 t^{5}+15 t^{4}-24 t^{3}+21 t^{2}-10 t+2\right) q^{3}+O\left(q^{4}\right)\right), \\
& \widehat{Z}_{2}(1 / q, t)=q^{1 / 5}\left(\left(-t^{3}+2 t^{2}-t\right)+\left(2 t^{4}-5 t^{3}+5 t^{2}-3 t+1\right) q+\right. \\
& \left.+\left(-2 t^{5}+7 t^{4}-13 t^{3}+14 t^{2}-7 t+1\right) q^{2}+O\left(q^{3}\right)\right) .
\end{aligned}
$$


Now we can check (3.35) and (3.55) directly. From (3.31), or equivalently from (3.77), we have

$$
\begin{aligned}
& \widehat{Z}_{0}(q, t)=1+q+\left(2-t+t^{2}\right) q^{2}+\left(3-2 t+2 t^{2}\right) q^{3}+O\left(q^{4}\right), \\
& \widehat{Z}_{1}(q, t)=q^{1 / 5}\left(-1+(-2+t) q+\left(-3+2 t-2 t^{2}\right) q^{2}+\left(-5+4 t-3 t^{2}+t^{3}\right) q^{3}+O\left(q^{4}\right)\right), \\
& \widehat{Z}_{2}(q, t)=q^{4 / 5}\left((1-t) q+\left(1-2 t+t^{2}\right) q^{2}+O\left(q^{3}\right)\right) .
\end{aligned}
$$

And from (3.31) we have:

$$
\begin{aligned}
\mathcal{I}(q, t)=\left(1-\frac{1}{t^{3}}\right)+\left(-1+\frac{1}{t}+\frac{1}{t^{2}}-\frac{1}{t^{3}}\right) q+ & \left(t^{2}-2+\frac{1}{t}-\frac{1}{t^{3}}+\frac{1}{t^{4}}\right) q^{2}+ \\
& +\left(\frac{1}{t}-\frac{1}{t^{2}}-\frac{1}{t^{5}}+\frac{1}{t^{6}}\right) q^{3}+O\left(q^{4}\right) .
\end{aligned}
$$

Then, one can check explicitly that indeed

$$
\mathcal{I}(q, t)=\widehat{Z}_{0}(q, t) \cdot 2 \widehat{Z}_{0}(1 / q, 1 / t)+\widehat{Z}_{1}(q, t) \widehat{Z}_{1}(1 / q, 1 / t)+\widehat{Z}_{2}(q, t) \widehat{Z}_{2}(1 / q, 1 / t) .
$$

Similarly, the topologically twisted index,

$$
\mathcal{I}_{\text {top }}(q, t)=1-t^{3}
$$

can indeed be decomposed as

$$
\mathcal{I}_{\text {top }}(q, t)=\widehat{Z}_{0}(q, t) \cdot 2 \widehat{Z}_{0}(1 / q, t)+\widehat{Z}_{1}(q, t) \widehat{Z}_{1}(1 / q, t)+\widehat{Z}_{2}(q, t) \widehat{Z}_{2}(1 / q, t) .
$$

\subsubsection{Positivity of coefficients}

In [26], it was shown that, up to an overall \pm sign, the refined homological blocks (with or without contribution from the Cartan component of the adjoint chiral) have the following positivity property:

$$
\begin{aligned}
\pm \widehat{Z}_{a}(q,-t) & \in q^{\Delta_{a}} \mathbb{Z}_{+}[t][[q]] \\
\pm(-q t ; q)_{\infty} \widehat{Z}_{a}(q,-t) & \in q^{\Delta_{a}} \mathbb{Z}_{+}[t][[q]]
\end{aligned}
$$

so that it is naturally to conjecture that $\pm \widehat{Z}_{a}(q,-t)$ actually coincides with the Poincaré polynomial of the underlying doubly graded homology (after a shift in overall degrees),

$$
\mathcal{H}_{a}\left[M_{3}\right]=\bigoplus_{\substack{i \in \mathbb{Z}+\Delta_{a} \\ j \in \mathbb{Z}}} \mathcal{H}_{a}^{i, j} .
$$

This is equivalent to the statement that the triply graded homology (refined by the flavor charge) is supported only on the "diagonal"

$$
\mathcal{H}_{a}^{i, j ; \ell}=\delta_{j, \ell} \mathcal{H}_{a}^{i, j} .
$$

Since we have advertised that the superconformal/twisted index and their categorifications are "better-behaved," one may ask whether they have a similar positivity property. The answer is affirmative. 
Superconformal index. In contrast to the homological blocks, the coefficients of $\mathcal{I}(q, t)$ are not all positive/negative and there does not seem to be an easy change of variables which achieves that (contrary to what happend for $\widehat{Z}_{a}(q, t)$, see [26]). However, there seem to be an easy factor which makes them positive. In particular, in the case of $G=S U(2)$ and $p>1$, one observes that

$$
\mathcal{I}_{T[L(p, 1), S U(2)]}(q, t)=P(q, t) \cdot\left(t^{-1} ; q\right)_{\infty} \cdot(1-q t)
$$

where

$$
P(q, t) \in \mathbb{Z}_{+}[t][[q]]
$$

is a positive power series. So, naively one can expect that the spectrum of BPS operators of $T[L(p, 1), S U(2)]$ is given by cohomology

$$
\mathcal{H}_{\mathrm{BPS}}=H^{*}\left(\mathcal{H}_{P} \otimes \mathcal{H}_{\mathrm{Fermi}}, d\right)
$$

for some differential $d$, where $\mathcal{H}_{P}$ categorifies $P(q, t)$ and $\mathcal{H}_{\text {Fermi }}$ is the space generated by a tower of fermions with the index $\left(t^{-1} ; q\right)_{\infty}$ and one single fermions with index $(1-t q)$. And one would expect the tower is related to $\bar{\psi}_{+}^{3}$, the Cartan component of $\bar{\psi}_{+}$in the (anti-)chiral multiplet, and its derivatives.

Twisted index. The twisted index also shares this positivity property, which can be understood in greater depth. For $T[L(p, 1) ; G]$ with large $p$, we know from the discussion in section 3.2.2 that

$$
\mathcal{H}_{\mathrm{tw}-\mathrm{BPS}}^{\bullet}=H^{\bullet}(G)=\left(\Lambda^{\bullet} \mathfrak{g}\right)^{G} .
$$

Then the equivariant Euler characteristics will factorizes into a positive part times a simple factor,

$$
\mathcal{I}_{\text {top }}=\prod_{i}^{\mathrm{rk} G}\left(1-t^{2 m_{i}+1}\right)=(1-t)^{\mathrm{rk} G} \cdot \prod_{i}^{\mathrm{rk} G}\left(1+t+\ldots+t^{2 m_{i}}\right),
$$

where $m_{i}$ are exponents of the group $G$. At the level of homological invariants, one may be tempted to factor out the exterior algebra of the Cartan subalgebra $\mathfrak{t} \subset \mathfrak{g}$

$$
(\Lambda \mathfrak{g})^{G} \sim \Lambda \mathfrak{t} \cdot(\Lambda \mathfrak{g})^{G} / \Lambda \mathfrak{t} .
$$

However, the last quotient doesn't make sense as $\Lambda \mathfrak{t}$ is not a submodule of $(\Lambda \mathfrak{g})^{G}$ — this parallels the discussion for the superconformal index where the gauge-non-invariant operator $\bar{\psi}_{+}^{3}$ plays the role of $\mathfrak{t}$ here. Instead, one has to consider the Leray spectral sequence

$$
E_{2}^{\bullet \bullet \bullet}=H^{\bullet}\left(G / T, H^{\bullet}(T)\right) \Rightarrow H^{\bullet}(G)
$$

associated with the fibration

$$
T \rightarrow G \rightarrow G / T .
$$

The $E_{2}$ page factorizes as

$$
H^{\bullet}(T) \otimes H^{\bullet}(G / T)
$$


where $H^{\bullet}(T)$ is fermionic (generated by elements in $H^{1}(T)$ ) while $H^{\bullet}(G / T$ ) is bosonic (with only even-degree cohomology groups). But the differentials in the spectral sequence are no longer trivial. And this is the reason that we expect (3.92) as opposed to a direct factorization of $\mathcal{H}_{\mathrm{BPS}}$.

A summary. Up to now, we have encountered several positivity-related phenomena, similar but each with its own flavor, and it may be worthwhile to summarize and compare.

1. Positivity of homological blocks $\widehat{Z}_{a}$. Besides overall signs, all homological blocks are positive in variables $q$ and $-t$. This is likely due to "homological-flavor locking" the homological degree is not independent from the flavor degree and one completely determines the other, $c f$. [26, sec.3.4]. We have already illustrated how this happens for $T\left[S^{3}\right]$ in section 3.1, and expect this to be a general phenomena. In fact, such behavior was already observed for homological invariants of knot $[19,34,51]$.

2. Positivity of the superconformal index $\mathcal{I}$ and twisted index $\mathcal{I}_{\text {top }}$. After factorizing out a "fermionic factor," all coefficients appearing in the two indices will be positive. This may seems to be qualitatively different from 1 , where we have positivity in $(q,-t)$ variable as opposed to $(q, t)$. However, the example of $\mathcal{I}_{\text {top }}(T[L(p, 1)])$ with large $p$ suggests that 1 and 2 are intimately related. Namely, from (3.94), the twisted index in this case is

$$
\mathcal{I}_{\text {top }}=P_{-t}(G)=\prod_{i}^{\mathrm{rk} G}\left(1-t^{2 m_{i}+1}\right)=(1-t)^{\mathrm{rk} G} \cdot(\text { positive polynomial })
$$

where one sees that the third quantity is positive in $-t$ via "homological-flavor locking", while removal of a factor will achieve strict positivity in the fourth quantity.

3. Positivity of " $\beta$-reduced" homological blocks $\widehat{Z}_{a}^{(\beta)}$ and indices $\mathcal{I}^{(\beta)}, \mathcal{I}_{\text {top }}^{(\beta)}$. For $\mathcal{I}^{(\beta)}$ and $\mathcal{I}_{\text {top }}^{(\beta)}$, their positivity directly follows from 2 by setting $t=q^{\beta}$. On the other hand, positivity for $\widehat{Z}_{a}^{(\beta)}$ is not a priori obvious and require non-trivial cancellations that also involve the $q$-degree. This hints at the non-trivial role played by the $q$-grading in the "homological-flavor locking."

\subsubsection{Comparison with refined CS}

Let $R_{n}$ denote by a representation of $S U(2)$ of dimension $n$. The Hilbert space of the refined Chern-Simons theory [19] on $T^{2}$ is the same as in the unrefined case. Namely, it is generated by integral representations of the affine Kac-Moody algebra at "bare" level $k-2$, which in 
our notations ${ }^{30}$ have $n=1, \ldots, k-1$ :

$$
\mathcal{H}_{T^{2}}=\bigoplus_{n=1}^{k-1} \mathbb{C}\left|R_{n}\right\rangle
$$

Let

$$
q=e^{\frac{2 \pi i}{k+2 \beta}}, \quad \text { and } \quad t=q^{\beta} .
$$

The $T$ and $S$ matrices read (up to simple $q, t$-independent phase factors)

$$
\begin{gathered}
T_{n m} \equiv\left\langle R_{n}|T| R_{m}\right\rangle=\delta_{n m} \cdot q^{\frac{n^{2}}{4}} t^{\frac{n}{2}+\frac{\beta}{4}} \\
S_{n m} \equiv\left\langle R_{n}|S| R_{m}\right\rangle=d_{R_{n}}(t) M_{R_{m}}\left(t q^{n}\right)
\end{gathered}
$$

where the refined quantum dimension $d_{R_{n}}$ is given by

$$
d_{R_{n}}(t q)=\frac{1}{(2 k+4 \beta)^{1 / 2}} \prod_{m=0}^{\beta}\left(q^{-(m+1) / 2} t^{-1 / 2}-q^{(m+1) / 2} t^{1 / 2}\right) M_{R_{n}}(t q)
$$

and the $S U(2)$ Macdonald polynomials are given by ${ }^{31}$

$$
M_{R_{n}}(x)=\sum_{j=0}^{n-1} x^{j-n / 2+1 / 2} \prod_{i=1}^{j} \frac{[n-i]_{q}[i+\beta]_{q}}{[n-i+\beta]_{q}[i]_{q}},
$$

which at the special value of the argument simplifies to

$$
M_{R_{n}}(t q)=\prod_{m=0}^{\beta} \frac{q^{-(m+n) / 2} t^{-1 / 2}-q^{(m+n) / 2} t^{1 / 2}}{q^{-(m+1) / 2} t^{-1 / 2}-q^{(m+1) / 2} t^{1 / 2}} .
$$

The scalar product on $\mathcal{H}_{T^{2}}$ is given by

$$
\begin{gathered}
\left\langle R_{n} \mid R_{m}\right\rangle=g_{n} \delta_{n m}, \\
g_{n}=\prod_{m=0}^{\beta} \frac{q^{-(n+m) / 2} t^{-1 / 2}-q^{(n+m) / 2} t^{1 / 2}}{q^{-(n-m) / 2} t^{-1 / 2}-q^{(n-m) / 2} t^{1 / 2}} .
\end{gathered}
$$

${ }^{30}$ Our notations slightly differ from those in [19]:

$$
\begin{gathered}
k_{\text {here }}=k_{\mathrm{AS}}+2, \\
\beta_{\text {here }}=\beta_{\mathrm{AS}}-1, \\
t_{\text {here }}=t_{\mathrm{AS}} / q .
\end{gathered}
$$

${ }^{31}$ As usual, $q$-numbers are defined as

$$
[n]_{q} \equiv \frac{q^{n / 2}-q^{-n / 2}}{q^{1 / 2}-q^{-1 / 2}} .
$$


The refined CS partition function on $M_{3}=L(p, 1)$ is then given by

$$
\begin{aligned}
& Z_{S U(2)}^{\text {ref. } C S}[L(p, 1)]=\sum_{n=1}^{k-1} g_{n}^{-1}\left(S_{1 n}\right)^{2}\left(T_{n n}\right)^{-p} \\
& \quad=(2 k+4 \beta)^{-1} \sum_{n=1}^{k-1} q^{-\frac{p n^{2}}{4}-\frac{p n \beta}{2}-\frac{p \beta^{2}}{4}} \prod_{j=0}^{\beta}\left(q^{-\frac{n+j+\beta}{2}}-q^{\frac{n+j+\beta}{2}}\right)\left(q^{-\frac{n-j+\beta}{2}}-q^{\frac{n-j+\beta}{2}}\right) .
\end{aligned}
$$

Changing the summation variable from $n$ to $m=n+\beta$ gives

$$
\begin{aligned}
(2 k+4 \beta) Z_{S U(2)}^{\text {ref. CS }}[L(p, 1)] & =\sum_{m=\beta+1}^{k+\beta-1} q^{-\frac{p m^{2}}{4}} \prod_{j=0}^{\beta}\left(q^{-\frac{m+j}{2}}-q^{\frac{m+j}{2}}\right)\left(q^{-\frac{m-j}{2}}-q^{\frac{m-j}{2}}\right) \\
= & \frac{(-1)^{\beta} q^{-\frac{\beta(\beta+1)}{2}}}{2} \sum_{m \in \mathbb{Z}_{2(k+2 \beta)}} q^{-\frac{p m^{2}}{4}} \prod_{j=0}^{\beta}\left(1-q^{j+m}\right)\left(1-q^{j-m}\right),
\end{aligned}
$$

where we have extended the range of summation using the parity and vanishing properties of the summand. Using the Gauss sum reciprocity formula (which is a particular case of (A.8))

$$
\sum_{m \in \mathbb{Z}_{2(k+2 \beta)}} q^{-\frac{p m^{2}}{4}} \cdot q^{\frac{n \ell}{2}}=\frac{e^{-\frac{\pi i}{4}}(2 k+4 \beta)^{1 / 2}}{|p|^{1 / 2}} \sum_{a \in \mathbb{Z}_{p}} e^{2 \pi i(k+2 \beta) \frac{a^{2}}{p}} \cdot e^{2 \pi i \frac{a \ell}{p}} \cdot q^{\frac{\ell^{2}}{4 p}}
$$

we can rewrite (3.112) as follows:

$$
\begin{aligned}
& (2 k+4 \beta)^{1 / 2} Z_{S U(2)}^{\text {ref. CS }}[L(p, 1)]= \\
& \quad=t^{-\frac{\beta+1}{2}} \sum_{a, b \in \mathbb{Z}_{p} / \mathbb{Z}_{2}} e^{2 \pi i(k+2 \beta) \operatorname{CS}(a)} \cdot S_{a b} \cdot \frac{1}{\left|\mathcal{W}_{b}\right|} \mathcal{L}_{b}^{(p)}\left[\prod_{j=0}^{\beta}\left(1-q^{j} z^{2}\right)\left(1-q^{j} z^{-2}\right)\right]
\end{aligned}
$$

where, again, we drop the overall phase factor and CS $(a)$ is the CS invariant of a flat connection $a$. As before, the $S$-transform is given by ${ }^{32}$

$$
S_{a b}=\frac{e^{2 \pi i \frac{a b}{p}}+e^{-2 \pi i \frac{a b}{p}}}{\left|\mathcal{W}_{a}\right|}
$$

and $\mathcal{L}_{b}^{(p)}$ is the "Laplace transform":

$$
\begin{aligned}
\mathcal{L}_{b}^{(p)}: \mathbb{Z}[q] \otimes \mathbb{Z}\left[z^{2}, z^{-2}\right] & \longrightarrow \\
z^{2 \ell} & \longmapsto\left\{\begin{array}{cl}
q^{\ell^{2} / p}, & \ell=b \bmod p \\
0, & \text { otherwise }
\end{array}\right.
\end{aligned}
$$

\footnotetext{
${ }^{32}$ Notice, that this $S$-transform matrix is different from the $S$-matrix in refined Chern-Simons theory!
} 
Equivalently, it can be realized as follows:

$$
\mathcal{L}_{b}^{(p)}: \quad f(z) \longmapsto \int \frac{d z}{2 \pi i z} f\left(z^{-1}\right) \sum_{n \in p \mathbb{Z}+b} q^{n^{2} / p} z^{2 n} .
$$

Therefore we have

$$
(2 k+4 \beta)^{1 / 2} Z_{S U(2)}^{\text {ref. CS }}[L(p, 1)]=t^{-\frac{\beta+1}{2}} \sum_{a, b \in \mathbb{Z}_{p} / \mathbb{Z}_{2}} e^{2 \pi i(k+2 \beta) \operatorname{CS}(a)} \cdot S_{a b} \cdot \widehat{Z}_{b}^{(\beta)}(q)
$$

where $\widehat{Z}_{b}^{(\beta)}(q)$ are the same as homological blocks (3.60) obtained as the $D^{2} \times_{q} S^{1}$ partition function of $T[L(p, 1), S U(2)]$,

$$
\widehat{Z}_{b}^{(\beta)}(q)=\left.\frac{1}{\left|\mathcal{W}_{b}\right|} \int \frac{d z}{2 \pi i z} \frac{\left(z^{2} ; q\right)_{\infty}\left(z^{-2} ; q\right)_{\infty}}{\left(z^{2} t q ; q\right)_{\infty}\left(z^{-2} t q ; q\right)_{\infty}} \sum_{n \in p \mathbb{Z}+b} q^{n^{2} / p} z^{2 n}\right|_{t=q^{\beta}}
$$

Therefore, we just showed that (3.118) can be understood as a refined version of the decomposition (2.6) of the WRT invariant into homological blocks $\widehat{Z}_{a}(q)$.

$3.3 M_{3}=O(-p) \rightarrow \Sigma_{g}$

Now, we consider a class of three-manifolds $M_{3}$ that could be viewed as a natural generalization of $L(p, 1)$ - the total space of a degree- $(-p)$ circle bundle over a genus- $g$ Riemann surface. We expect the $3 \mathrm{~d} \mathcal{N}=2$ theory $T\left[M_{3}, S U(2)\right]$ to have the following Lagrangian description:

$$
T\left[M_{3}\right]=\begin{gathered}
\mathcal{N}=2 \text { level- } p \text { Chern-Simons term } \\
+ \text { adjoint chiral } \Sigma \text { with R-charge } 2 \\
+2 g \text { adjoint chirals }\left(\Phi_{i}, \widetilde{\Phi}_{i}\right)_{i=1}^{g} \text { with R-charge } 0 .
\end{gathered}
$$

When $p=0$, the theory has $\mathcal{N}=4$ supersymmetry and coincides with the mirror description of the $3 \mathrm{~d}$ reduction of $T\left[\Sigma_{g}\right][52]$. Having $p \neq 0$ results in $p$ units of $\mathrm{RR} 2$-form flux after reducing the M5-branes first on the circle fiber, which then give rise to a level- $p$ Chern-Simons term via D4-brane world-volume couplings to RR fields. The assignment of R-symmetry charges is consistent with the cubic superpotential coupling $\operatorname{Tr} \widetilde{\Phi_{i}} \Sigma \Phi_{i}$ and reproduces the the correct R-charge assignment for the Lens space theory $T[L(p, 1)]$ when $g=0$.

When $p=0$, the theory $T\left[M_{3}\right]$, considered as an $\mathcal{N}=2$ theory has $U(1)_{\beta} \times U S p(2 g)$ symmetry [52], where $U S p(2 g)$ rotates $2 g$ adjoint chirals and $U(1)_{\beta}$ is the anti-diagonal of $S U(2) \times S U(2)$ R-symmetry, with respect to which the fields $\Phi_{i}, \widetilde{\Phi}_{i}$ and $\Sigma$ have charges -1 , 0 and 1 respectively. ${ }^{33}$ When $p \neq 0$, the CS interaction does not break these symmetries, but the $U(1)_{t^{\prime}}$ R-symmetry of the $\mathcal{N}=4$ theory with $p=0$ will become the flavor symmetry

\footnotetext{
${ }^{33}$ This is because $\Phi_{i}$ and $\widetilde{\Phi}_{i}$ are combined into an $\mathcal{N}=4$ twisted hypermultiplet [52] and transform under the $S U(2) \times S U(2)$ in the same way as a vector multiplet. As a consequence, $U(1)_{\beta}$ only acts on $\Phi_{i}$, not $\widetilde{\Phi}_{i}$.
} 
$U(1)_{\beta}$ of the $\mathcal{N}=2$ theory with $p \neq 0$. For our purposes, it is more convenient to consider instead $U(1)_{\beta} \times U(g)$ maximal rank subgroup with the following charge assignments:

\begin{tabular}{c|cc} 
& $U(1)_{\beta}$ & $U(g)$ \\
\hline$\Sigma$ & 1 & $\mathbf{1}$ \\
$\Phi_{i}$ & -1 & $\mathbf{g}$ \\
$\widetilde{\Phi}_{i}$ & 0 & $\mathbf{g}$
\end{tabular}

\subsubsection{Homological blocks and WRT invariant}

Consider the partition function of $T\left[M_{3}\right]$ on $D^{2} \times S^{1}$ with the following $2 \mathrm{~d} \mathcal{N}=(0,2)$ boundary conditions (which generalize the boundary conditions for $T[L(p, 1)]$ that produce $\widehat{Z}_{a}(q, t)$ ):

- Neumann for $\mathcal{N}=2$ vector multiplet,

- Neumann for adjoint chiral $\Sigma$,

- Dirichlet for $g$ adjoint chirals $\Phi_{i}$,

- Neumann for $g$ adjoint chirals $\widetilde{\Phi}_{i}$,

- $3 \mathrm{~d}$ vector multiplet is coupled to modules of $\mathbb{Z}_{2}$-orbifolded $\sqrt{2 p} \mathbb{Z}$ lattice VOA by gauging its flavor symmetry. Note that when $p=0$ the boundary conditions preserve $2 \mathrm{~d} \mathcal{N}=$ $(2,2)$ supersymmetry. This cancels gauge anomaly inflow due to the level- $p$ CS term.

The $D^{2} \times{ }_{q} S^{1}$ partition then reads [29]

$$
\begin{aligned}
\widehat{Z}_{a}(q, t, u) \equiv Z_{T\left[M_{3}\right]}\left(D_{a}^{2} \times_{q} S^{1}\right)= & \frac{1}{\left|\mathcal{W}_{a}\right|} \int_{\gamma} \frac{d z}{2 \pi i z} \frac{\left(z^{ \pm 2} ; q\right)_{\infty}(q ; q)_{\infty}}{\left(z^{ \pm 2} t q ; q\right)_{\infty}(t q ; q)_{\infty}} \times \\
& \prod_{i=1}^{g} \frac{\left(z^{ \pm 2} t u_{i} q ; q\right)_{\infty}\left(t u_{i} q ; q\right)_{\infty}}{\left(z^{ \pm 2} u_{i} ; q\right)_{\infty}\left(u_{i} ; q\right)_{\infty}} \times \frac{\sum_{n \in p \mathbb{Z}+a} q^{n^{2} / p} z^{2 n}}{(q ; q)_{\infty}}
\end{aligned}
$$

The choice of the contour $\gamma$, which corresponds to a precise choice of the boundary condition for the vector multiplet (or, equivalently, Lagrangian submanifold in the complexified maximal torus of $S U(2))$, will be discussed below.

The WRT invariant of $M_{3}$ can be computed as follows in terms of $S$ and $T$ matrices $^{34}$ :

$$
Z_{S U(2)_{k}}^{\mathrm{CS}}\left[M_{3}\right]=\sum_{n=1}^{k-1}\left(S_{1 n}\right)^{2-2 g}\left(T_{n n}\right)^{-p}=\sum_{n \in \mathbb{Z}_{2 k}}^{\prime}\left(q^{n / 2}-q^{-n / 2}\right)^{2-2 g} q^{-\frac{p n^{2}}{4}}
$$

where we use notation such that matrix indices are "colors" (= dimensions of representations), $q=e^{\frac{2 \pi i}{k}}$ and a prime over the sum means that we skip singular terms. After applying the Gauss reciprocity formula

$$
\sum_{m \in \mathbb{Z}_{2 k}} q^{-\frac{p m^{2}}{4}} \cdot q^{\frac{n \ell}{2}}=\frac{e^{-\frac{\pi i}{4}}(2 k)^{1 / 2}}{|p|^{1 / 2}} \sum_{a \in \mathbb{Z}_{p}} e^{2 \pi i k \frac{a^{2}}{p}} \cdot e^{2 \pi i \frac{a \ell}{p}} \cdot q^{\frac{\ell^{2}}{4 p}},
$$

\footnotetext{
${ }^{34}$ We omit simple overall factors below.
} 
we arrive at

$$
Z_{S U(2)_{k}}^{\mathrm{CS}}\left[M_{3}\right]=\left.\sum_{a, b \in \mathbb{Z}_{p} / \mathbb{Z}_{2}} e^{2 \pi i k \operatorname{CS}(a)} \cdot S_{a b} \cdot \frac{1}{\left|\mathcal{W}_{b}\right|} \widehat{Z}_{b}(q)\right|_{q \rightarrow e^{\frac{2 \pi i}{k}}}
$$

where the $S$-matrix is given in (3.115) and, formally,

$$
\widehat{Z}_{a}(q)=\mathcal{L}_{a}^{(p)}\left[\left(z-z^{-1}\right)^{2-2 g}\right]
$$

with Laplace transform given in (3.116). To be precise, it can be computed using

$$
\widehat{Z}_{a}(q)=\frac{1}{\left|\mathcal{W}_{a}\right|} \cdot \mathrm{v} \cdot \mathrm{p} \cdot \int_{|z|=1}\left(z-z^{-1}\right)^{2-2 g} \sum_{n \in p \mathbb{Z}+a} q^{n^{2} / p} z^{2 n}
$$

as a series in $q$, convergent in the unit disk $|q|<1$. One can see that (3.127) coincides with the unrefined partition function of $T\left[M_{3}\right]$ on $D^{2} \times S^{1}$, cf. (3.122), with removed contribution of Cartan components of the adjoint chiral:

$$
\widehat{Z}_{a}(q)=\left.\widehat{Z}_{a}(q, t, u)(q t ; q)_{\infty} \prod_{i=1}^{g} \frac{\left(u_{i} ; q\right)_{\infty}}{\left(t u_{i} q ; q\right)_{\infty}}\right|_{t=1, u_{i}=1}
$$

The principal value contour prescription in (3.127) suggests that the contour $\gamma$ in (3.122) should be chosen as shown in Figure 3. Note, that the labels $a$ of homological blocks now correspond to the connected components of the space of abelian flat connections.

\subsubsection{Factorization of the superconformal index}

The superconformal index of $3 \mathrm{~d} \mathcal{N}=2$ theory (3.120) reads

$$
\begin{aligned}
\mathcal{I}_{T\left[M_{3}\right]}(q, t)= & \sum_{m \geq 0} \frac{1}{\left|\mathcal{W}_{m}\right|} \int_{|z|=1} \frac{d z}{2 \pi i z} z^{2 p m}\left(1-z^{ \pm 2} q^{m}\right) q^{-2 m(1-g)} t^{-2 m(1-g)} \times \\
& \frac{\left(z^{ \pm 2} q^{m} t^{-1} ; q\right)_{\infty}\left(t^{-1} ; q\right)_{\infty}}{\left(z^{ \pm 2} q^{m+1} t\right)_{\infty}(q t ; q)_{\infty}} \times \\
& \prod_{i=1}^{g} \frac{\left(z^{ \pm 2} u_{i}^{-1} q^{m+1} ; q\right)_{\infty}\left(u_{i}^{-1} q ; q\right)_{\infty}}{\left(z^{ \pm 2} u_{i} q^{m} ; q\right)_{\infty}\left(u_{i} ; q\right)_{\infty}} \cdot \frac{\left(z^{ \pm 2} t u_{i} q^{m+1} ; q\right)_{\infty}\left(t u_{i} q ; q\right)_{\infty}}{\left(z^{ \pm 2} t^{-1} u_{i}^{-1} q^{m} ; q\right)_{\infty}\left(t^{-1} u_{i}^{-1} ; q\right)_{\infty}} .
\end{aligned}
$$

Unlike in the case of Lens spaces $(g=0)$, now there is some ambiguity in the choice of the contour, because if $|t|=1$ there are poles at $|z|=1$. The natural physical choice assumes that $|q|<1$ and $\left|u_{i}\right|=\left|t^{-1} u_{i}^{-1}\right|=q^{\epsilon}$ with $\epsilon>0$, which corresponds to choosing an infinitesimally small R-charge for adjoint hypers. However, such choice of the contour is different from the one in (3.122) and (3.127), illustrated in Figure 3.

Next, we would like to show that

$$
\mathcal{I}_{T\left[M_{3}\right]}(q, t, u)=\sum_{a}\left|\mathcal{W}_{a}\right| \widehat{Z}_{a}(q, t, u) \widehat{Z}_{a}(1 / q, 1 / t, 1 / u)
$$




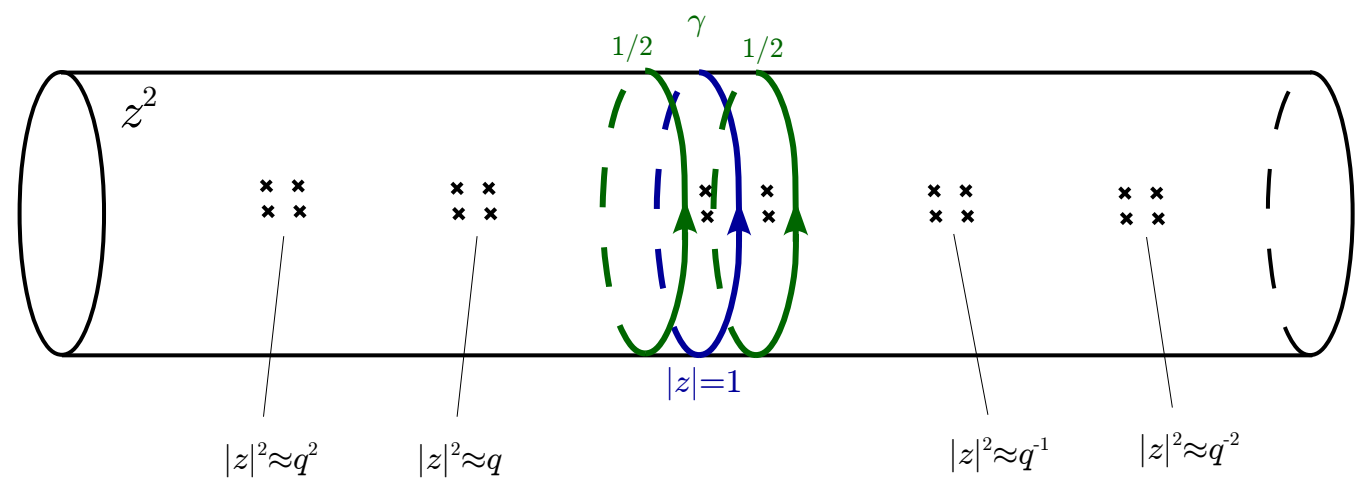

Figure 3: Pole structure of the integrands in (3.122) and (3.129). The green contour corresponds to a choice of the contour $\gamma$ such that in the $t \rightarrow 1$ limit it agrees with the principal-value prescription in (3.127) which reproduces the WRT invariant. The blue contour is the standard physical choice of contour for the superconformal index (3.129).

As before, the problem with checking it directly is that we only have the expression for $\widehat{Z}_{a}(q, t)$ which is valid inside a unit disc $|q|<1$, without any systematic way of analytically continuing it outside. Again, we can instead try to check (3.130) in a particular limit of flavor fugacities such that $\widehat{Z}_{a}(q, t)$ becomes a rational function and has canonical analytic continuation outside of $|q|<1$. However, one finds that there are several new technical complications compared to the case of Lens spaces $(g=0)$. First, there is an ambiguity in the choice of the contours (i.e. whether we encircle the poles at $z^{2}=t^{ \pm 1 / 2}$ or not), illustrated in Figure 3. Second, for $g>1$ the limit $u_{i} \rightarrow 1, t \rightarrow q^{\beta}$ with $\beta \in \mathbb{Z}_{\geq 0}$ does not give a rational function, but rather a lacunary function that cannot be analytically continued outside $|q|<1$ due to the Fabry gap theorem. In particular, one cannot repeat the trick with the cyclotomic expansion due to the absence of closed form expression for $t=1$.

However, one can avoid all these problems by taking the limit $u_{i} \rightarrow 1, t \rightarrow q^{\beta}$, with $\beta<0$. In this limit, all poles at $|z| \approx 1$ disappear and the contour ambiguity becomes irrelevant. Namely, for $\beta \in \mathbb{Z}_{-}$, let us define

$$
\left.\widehat{Z}_{a}^{(\beta)}(q) \equiv \widehat{Z}_{a}(q, t)(q t ; q)_{\infty} \prod_{i=1}^{g} \frac{\left(u_{i} ; q\right)_{\infty}}{\left(t u_{i} q ; q\right)_{\infty}}\right|_{t=q^{\beta}, u_{i}=1}
$$

and

$$
\left.\mathcal{I}^{(\beta)}(q) \equiv \mathcal{I}(q, t) \frac{(q t ; q)_{\infty}}{(1 / t ; q)_{\infty}} \prod_{i=1}^{g} \frac{\left(u_{i} ; q\right)_{\infty}\left(t^{-1} u_{i}^{-1} ; q\right)_{\infty}}{\left(u_{i}^{-1} q ; q\right)_{\infty}\left(t u_{i} q ; q\right)_{\infty}}\right|_{t=q^{\beta}, u_{i}=1}
$$

Then, one can check for various values of $g, p, \beta<0$ that indeed

$$
\mathcal{I}^{(\beta)}(q)=\sum_{a}\left|\mathcal{W}_{a}\right| \widehat{Z}_{a}^{(\beta)}(q) \widehat{Z}_{a}^{(\beta)}(1 / q)
$$

For example, let us take $g=2$ and $p=5$. Then, we have the following. 


$$
\begin{aligned}
& \beta=-1 \text { : } \\
& 2 \widehat{Z}_{0}^{(-1)}(q)=1, \quad \widehat{Z}_{1}^{(-1)}(q)=0, \quad \widehat{Z}_{2}^{(-1)}(q)=0 . \\
& 2 \mathcal{I}^{(-1)}(q)=1 \text {. } \\
& \beta=-2 \text { : } \\
& 2 \widehat{Z}_{0}^{(-2)}(q)=q^{-2}+1, \quad \widehat{Z}_{1}^{(-2)}(q)=q^{-4 / 5}, \quad \widehat{Z}_{2}^{(-2)}(q)=0 . \\
& 2 \mathcal{I}^{(-2)}(q)=4+q^{2}+\frac{1}{q^{2}} \text {. } \\
& \beta=-3: \\
& 2 \widehat{Z}_{0}^{(-3)}(q)=\frac{1}{q^{6}}+\frac{1}{q^{4}}+\frac{2}{q^{3}}+\frac{1}{q^{2}}+1, \widehat{Z}_{1}^{(-3)}(q)=-\frac{(q+1)^{2}\left(q^{2}-q+1\right)}{q^{24 / 5}}, \widehat{Z}_{2}^{(-3)}(q)=\frac{1}{q^{11 / 5}} \text {. } \\
& 2 \mathcal{I}^{(-3)}(q)=q^{6}+\frac{1}{q^{6}}+4 q^{4}+\frac{4}{q^{4}}+8 q^{3}+\frac{8}{q^{3}}+5 q^{2}+\frac{5}{q^{2}}+8 q+\frac{8}{q}+18 .
\end{aligned}
$$

And indeed one can verify that in each case

$$
\mathcal{I}^{(\beta)}(q)=2 \widehat{Z}_{0}^{(\beta)}(q) \widehat{Z}_{0}^{(\beta)}(1 / q)+\widehat{Z}_{1}^{(\beta)}(q) \widehat{Z}_{1}^{(\beta)}(1 / q)+\widehat{Z}_{2}^{(\beta)}(q) \widehat{Z}_{2}^{(\beta)}(1 / q) .
$$

Note, when $g>1$ the fundamental group $\pi_{1}\left(M_{3}\right)$ is non-abelian and $\mathcal{M}_{\text {flat }}\left(M_{3}, S L(2, \mathbb{C})\right)$ has irreducible flat connections. We confirmed, however, that the WRT invariant and the superconformal index both have a natural decomposition into contributions labelled by connected components of abelian flat connections. ${ }^{35}$ This "completeness" of the homological blocks is a striking and highly non-trivial phenomenon. Its physical origin was already discussed in section 2 from the viewpoint of resurgent analysis and charges which label BPS states / enumerative invariants in the physical system (2.13). It would be interesting to explore it further in various duality frames and from other vantage points.

\subsubsection{Comparison with refined CS}

In the notations of section 3.2.6, the partition function of refined Chern-Simons theory on $M_{3}=O(-p) \rightarrow \Sigma_{g}$ reads [19]:

$$
\begin{aligned}
Z_{S U(2)}^{\text {ref. CS }}\left[M_{3}\right] & =\sum_{n=1}^{k-1} g_{n}^{g-1}\left(S_{1 n}\right)^{2-2 g}\left(T_{n n}\right)^{-p}= \\
& =\sum_{n=1}^{k-1} q^{-\frac{p n^{2}}{4}-\frac{p n \beta}{2}-\frac{p \beta^{2}}{4}} \prod_{j=0}^{\beta}\left(q^{-\frac{n+j+\beta}{2}}-q^{\frac{n+j+\beta}{2}}\right)^{1-g}\left(q^{-\frac{n-j+\beta}{2}}-q^{\frac{n-j+\beta}{2}}\right)^{1-g} .
\end{aligned}
$$

By repeating manipulations in section 3.2.6, we arrive at (up to a simple overall factor)

$$
Z_{S U(2)}^{\text {ref. CS }}\left[M_{3}\right]=\sum_{a, b \in \mathbb{Z}_{p} / \mathbb{Z}_{2}} e^{2 \pi i(k+2 \beta) \operatorname{CS}(a)} \cdot S_{a b} \cdot \widehat{Z}_{b}^{(\text {ref.CS} ; \beta)}(q)
$$

\footnotetext{
${ }^{35}$ It is important to keep in mind that the terms in this decomposition are not merely contributions of the corresponding connected components of abelian flat connections to the path integral, but rather contain contributions of irreducible flat connections as well [22]. In other words, even though the sum in the decomposition runs only over abelian flat connections, all flat connections are accounted properly, reducible and irreducible; no one is left behind.
} 
with

$$
\begin{aligned}
\left|\mathcal{W}_{a}\right| \widehat{Z}_{a}^{(\text {ref.CS } \beta \beta)}(q)=\text { v.p. } & \int_{|z|=1} \frac{d z}{2 \pi i z}\left(z^{ \pm 2} ; q\right)_{\beta+1}^{1-g} \sum_{n \in p \mathbb{Z}+a} q^{n^{2} / p} z^{2 n}= \\
& =\text { v.p. }\left.\int_{|z|=1} \frac{d z}{2 \pi i z}\left(\frac{\left(z^{ \pm 2} ; q\right)_{\infty}}{\left(z^{ \pm 2} q t ; q\right)_{\infty}}\right)^{1-g} \sum_{n \in p \mathbb{Z}+a} q^{n^{2} / p} z^{2 n}\right|_{t=q^{\beta}}
\end{aligned}
$$

where "v.p." stand for principal value integration. This indeed agrees with the partition function of $T\left[M_{3}\right]$ on $D^{2} \times S^{1}, c f$. (3.122),

$$
\widehat{Z}_{a}^{(\text {ref.CS } ; \beta)}(q)=\left.\widehat{Z}_{a}(q, t, u) \cdot(q t ; q)_{\infty} \prod_{i=1}^{g} \frac{\left(u_{i} ; q\right)_{\infty}}{\left(t u_{i} q ; q\right)_{\infty}}\right|_{t=q^{\beta}, u_{i}=1} .
$$

\section{$3.4 M_{3}=$ plumbed}

Now we move to consider another very large class of 3-manifolds $M_{3}(\Gamma)$ associated to a plumbing graph $\Gamma$. There are various ways to define $M_{3}$ for a given $\Gamma$. One of them is to say that $M_{3}$ is obtained by a Dehn surgery on the corresponding link $\mathcal{L}(\Gamma)$ of unknots (see Figure 4). For simplicity, we also assume that $\Gamma$ is connected. Such class of 3-manifolds contains Seifert fibrations over $S^{2}$, which correspond to star-shaped plumbing graphs. Denote by $L$ the number of vertices of $\Gamma$. It is equal to the number of components of the link $\mathcal{L}(\Gamma)$. We will also need $L \times L$ linking matrix of $\mathcal{L}(\Gamma)$ which will be denoted by $M$ :

$$
M_{v_{1}, v_{2}}=\left\{\begin{array}{l}
1, \quad v_{1}, v_{2} \text { connected, } \\
a_{v}, v_{1}=v_{2}=v, \\
0, \text { otherwise. }
\end{array} \quad v_{i} \in \text { Vertices of } \Gamma \cong\{1, \ldots, L\} .\right.
$$
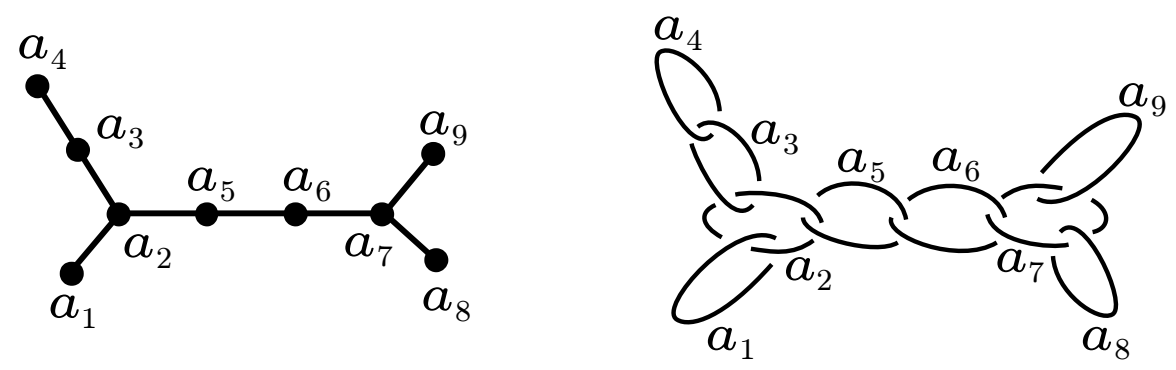

Figure 4: An example of a plumbing graph $\Gamma$ (left) and the corresponding link $\mathcal{L}(\Gamma)$ of framed unknots in $S^{3}$ (right). The associated 3-manifold $M_{3}(\Gamma)$ can be constructed by performing a Dehn surgery on $\mathcal{L}(\Gamma)$.

The first homology group of $M_{3}(\Gamma)$ is given by the cokernel (over $\mathbb{Z}$ ) of the linking matrix:

$$
H_{1}\left(M_{3}, \mathbb{Z}\right) \cong \text { Coker } M=\mathbb{Z}^{L} / M \mathbb{Z}^{L}
$$


In what follows we assume for simplicity that $M_{3}(\Gamma)$ is a rational homology sphere $\left(b_{1}\left(M_{3}\right)=\right.$ 0 ), which implies that $\Gamma$ has no loops (i.e. it is a tree). The linking form on $H_{1}\left(M_{3}, \mathbb{Z}\right)$ is then given by

$$
\ell k(a, b)=\left(a, M^{-1} b\right) \quad \bmod \mathbb{Z}, \quad a, b \in \mathbb{Z}^{L} / M \mathbb{Z}^{L} .
$$

Different plumbing graphs can give homeomorphic 3-manifolds $-M_{3}(\Gamma) \cong M_{3}\left(\Gamma^{\prime}\right)$ iff $\Gamma$ and $\Gamma^{\prime}$ can be connected by a sequence of 3 d Kirby moves, as shown in Figure $5^{36}$.
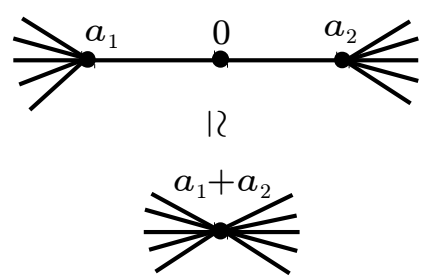

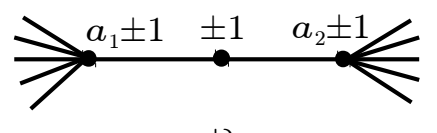

12

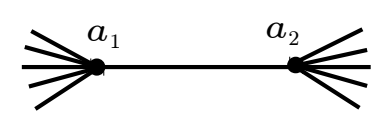

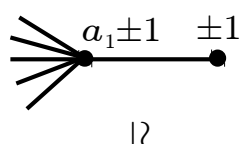

12

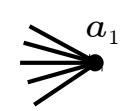

Figure 5: 3d Kirby moves that relate plumbing graphs which result in homeomorphic 3-manifolds.

The theory $T\left[M_{3}, S U(2)\right]$ can in principle be obtained by combining together the following $3 \mathrm{~d}$ theories (see e.g. [45]):

- edge: $S$-duality wall in $\mathcal{N}=4 S U(2)$ super-Yang-Mills (i.e. theory $T[S U(2)]$ ),

- 2 -valent vertex: $T^{a}$-duality wall = "supesymmetric Chern-Simons term at level $a, "$

- $n$-valent vertex: $T[$ (sphere with $n$ holes $\left.) \times S^{1}\right]=n$ copies of $T[S U(2)]$ gauged together.

\subsubsection{Homological blocks $\widehat{Z}_{a}$}

The homological blocks can be calculated by the following formula (see appendix A for a derivation):

$$
\begin{array}{r}
\widehat{Z}_{b}(q)=q^{-\frac{3 L+\sum_{v} a_{v}}{4}} \cdot \text { v.p. } \int_{\left|z_{v}\right|=1} \prod_{v} \in q_{\text {Vertices }} \frac{d z_{v}}{2 \pi i z_{v}}\left(z_{v}-1 / z_{v}\right)^{2-\operatorname{deg}(v)} \cdot \Theta_{b}^{-M}(z) \\
\equiv q^{-\frac{3 L+\sum_{v} a_{v}}{4}} \cdot \text { v.p. } \int_{\left|z_{v}\right|=1} \prod_{v \in \text { Vertices }} \frac{d z_{v}}{2 \pi i z_{v}}\left(z_{v}-1 / z_{v}\right)^{2} \times \\
\quad \times \prod_{\left(v_{1}, v_{2}\right) \in \text { Edges }} \frac{1}{\left(z_{v_{1}}-1 / z_{v_{1}}\right)\left(z_{v_{2}}-1 / z_{v_{2}}\right)} \cdot \Theta_{b}^{-M}(z)
\end{array}
$$

where $\operatorname{deg}(v)$ is the degree of a vertex $v$ and, as before, "v.p." means taking principle value integral (i.e. take half-sum of contours "dodging" poles from both sides) and $\Theta_{b}^{-M}(x)$ is the

\footnotetext{
${ }^{36}$ There is also a move which relates a connected graph to a disconected one. For the sake of brevity we don't discuss the case of disconnected plumbing graphs here.
} 
theta function of the lattice corresponding to minus the linking form $M$ given by

$$
\Theta_{b}^{-M}(x)=\sum_{\ell \in 2 M \mathbb{Z}^{L}+b} q^{-\frac{\left(\ell, M^{-1} \ell\right)}{4}} \prod_{i=1}^{L} x_{i}^{\ell_{i}} .
$$

Remarks on (3.145) :

1. In the special case when the plumbing graph has just one vertex, the expression for the homological block reduces to (3.60) with $\beta=0$.

2. Generically it is well-defined only if $M$ is negative definite, i.e. $M_{3}(\Gamma)$ is a link of a normal singularity, with $\Gamma$ being a resolution graph of the singularity.

3. The formula (3.145) defines homological blocks $\widehat{Z}_{a}(q)$ in terms of a plumbing graph $\Gamma$, but one can show that they are invariant under Kirby moves (Figure 5) acting on $\Gamma$. Therefore, $\widehat{Z}_{a}(q)$ actually depends only on the homeomorphism class of $M_{3}(\Gamma)$, and is indeed a topological invariant. A priori this is not obvious, since (3.145) has been obtained (see Appendix A) as an analytic continuation of WRT invariant, which, in principle, does not have to be unique.

4. Even though the formula has the structure of the "index of a quiver theory", it is not clear how to properly realize it as a partition function of $T\left[M_{3}(\Gamma)\right]$ on $D_{b}^{2} \times S^{1}$ with some boundary condition.

5. Suppose $M<0$. Then $\widehat{Z}_{a}(q)$ is a well-defined series in $q$ convergent in the unit disk $|q|<1$. Unless $M_{3}$ is a Lens space, $\widehat{Z}_{a}(q)$ does not admit analytic continuation (in the usual sense) outside of $|q|<1$ due to Fabry gap theorem (because the powers of $q$ with non-zero coefficients grow quadratically).

6. We have

$$
\widehat{Z}_{b}(q) \in 2^{-c} q^{\Delta_{b}} \mathbb{Z}[[q]]
$$

where

$$
\begin{gathered}
c \in \mathbb{Z}_{+}, \quad c \leq L, \\
b \in(2 \text { Coker } M+\delta) / \mathbb{Z}_{2} \stackrel{\text { Set }}{\cong} H_{1}\left(M_{3}, \mathbb{Z}\right) / \mathbb{Z}_{2}, \\
\delta \in \mathbb{Z}^{L} / 2 \mathbb{Z}^{L}, \quad \delta_{v}=\operatorname{deg} v \bmod 2 . \\
\Delta_{b}=-\frac{3 L+\sum_{v} a_{v}}{4}-\max _{\ell \in 2 M \mathbb{Z}^{L}+b} \frac{\left(\ell, M^{-1} \ell\right)}{4} \in \mathbb{Q}
\end{gathered}
$$

where $\mathbb{Z}_{2}$ acts as

$$
b \mapsto-b
$$

and is a symmetry of (3.145). 


\section{Example 1:}

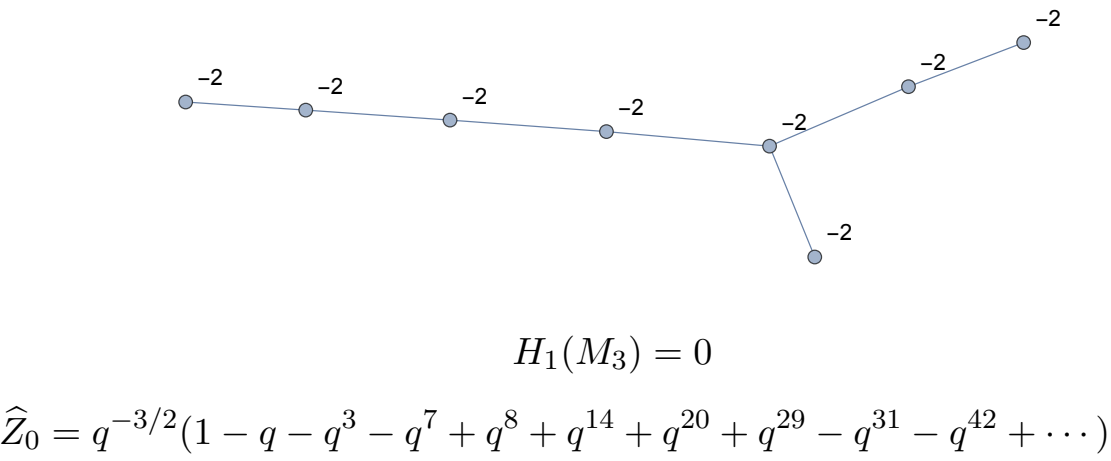

\section{Example 2:}

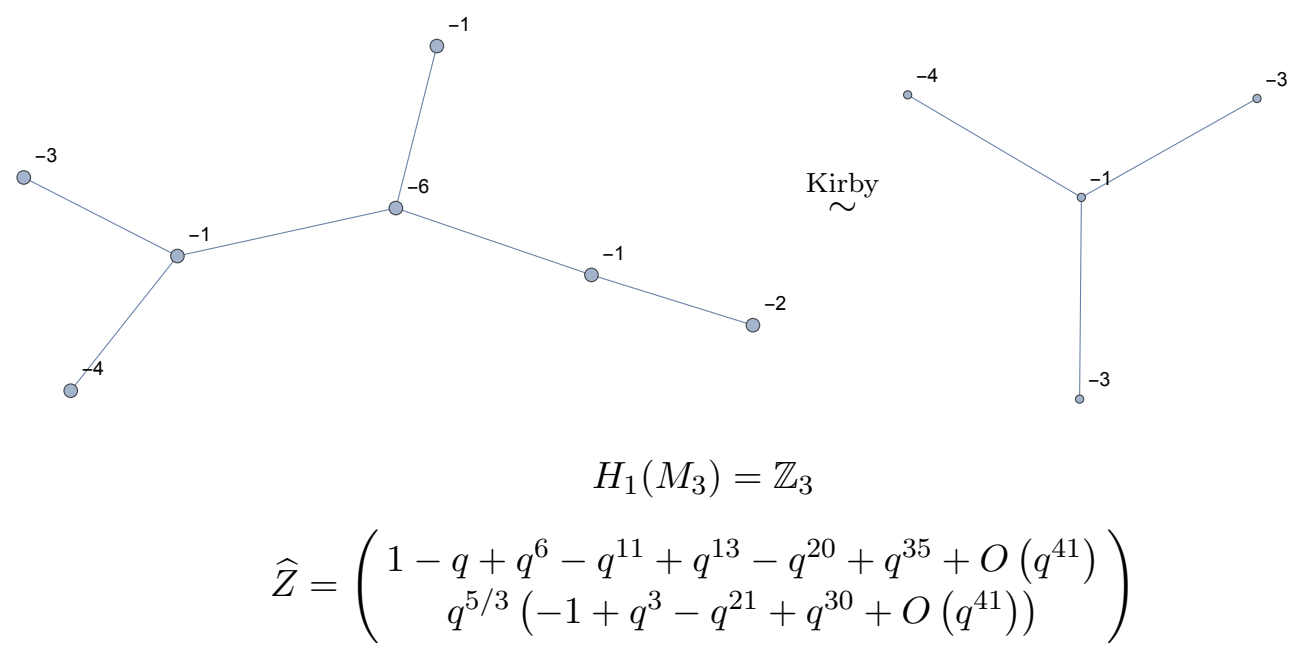

Example 3: (non-Seifert)

$$
\widehat{Z}=\frac{1}{4}\left(\begin{array}{c}
q^{7 / 26}\left(4+q+2 q^{3}-2 q^{4}-2 q^{6}-3 q^{7}-2 q^{8}-2 q^{10}-q^{11}-2 q^{13}-4 q^{14}-2 q^{15}-4 q^{16}+O\left(q^{18}\right)\right) \\
q^{-7 / 26}\left(-3-3 q^{2}+2 q^{4}-2 q^{5}+4 q^{7}+2 q^{8}+2 q^{9}+2 q^{10}+4 q^{12}+4 q^{13}+4 q^{14}+21^{15}+O\left(q^{18}\right)\right) \\
q^{-11 / 26}\left(-1+2 q-2 q^{2}+4 q^{3}-3 q^{6}-2 q^{7}+4 q^{8}-2 q^{9}+2 q^{11}+2 q^{13}+q^{14}-2 q^{16}+O\left(q^{17}\right)\right) \\
q^{-5 / 26}\left(2+2 q^{2}-q^{3}-3 q^{5}-2 q^{6}+2 q^{7}-4 q^{8}-2 q^{10}-2 q^{11}-2 q^{12}-2 q^{13}-5 q^{15}-2 q^{16}+O\left(q^{17}\right)\right) \\
q^{-15 / 26}\left(-1-2 q+2 q^{2}-q^{4}+2 q^{6}-2 q^{7}+2 q^{8}+4 q^{10}+2 q^{12}+2 q^{13}+2 q^{15}+O\left(q^{17}\right)\right)
\end{array}\right)
$$




\subsubsection{Problems on the $T\left[M_{3}\right]$ side}

One may wish to carry out the categorification program for $M_{3}(\Gamma)$ as well. However, it is not obvious how to write a correct expression for superconformal or topologically twisted index of the $3 \mathrm{~d} \mathcal{N}=2$ theory $T\left[M_{3}\right]$ because the UV description with $\mathcal{N}=2$ supersymmetry of its certain components (see the paragraph above Section 3.4.1) is not known. In particular, there is no known Lagrangian description for half-BPS T-duality walls in $\mathcal{N}=4$ super-Yang-Mills theory, and understanding the theory $T\left[M_{3}(\Gamma)\right]$ and its Hilbert space poses a challenge. We hope to study this problem in the future.

Let us note that a certain expression of the superconformal index of $T\left[M_{3}\right]$, with $M_{3}$ being an $S L\left(2, \mathbb{Z}\right.$ )-mapping class torus of punctured $T^{2}$ (which, without a puncture, would be homeomorphic to $M_{3}(\Gamma)$ with circular plumbing $\left.\Gamma\right)$ was considered in [53,54]. In particular, there it was checked that it was invariant under the first two of the Kirby moves in Figure 5 that correspond to relations in the $S L(2, \mathbb{Z})$ group. However, as it was argued in [54] it coincides with the result given by triangulation decomposition, as in $[55,56]$, and therefore, if interpreted as the partition function of the complex Chern-Simons theory, it misses contributions of the reducible flat connections [46]. The latter as we have seen, play a major role for categorification of WRT invariant. Also, in principle one can extend their prescription for calculating superconformal indices associated with plumbing graphs with vertices of degree other than two, by requiring invariance under the third Kirby move in Figure 5.

\section{Adding knots and links into $M_{3}$}

The goal of this section is to incorporate knots and links in a non-trivial 3-manifold $M_{3}$ and produce the corresponding homological invariants. In particular, it will give us the very first examples where both knots and 3-manifolds are non-trivial.

The physical setup that we use to make concrete predictions for the new homological invariants $\mathcal{H}_{a}\left[M_{3} ; K, \mathcal{R}\right]$ was already briefly mentioned in (2.56). As before, $K$ denotes a knot in $M_{3}$ while $\mathcal{R}$ denotes a representation (color) of CS gauge group $G$. The case of links is similar and involves assigment of color to each component of the link. The space $\mathcal{H}_{a}\left[M_{3} ; K, \mathcal{R}\right]$ can be explored from a number of vantage points and in a variety of duality frames (all of which lead to the same result). In particular, from the vantage point of the theory $T\left[M_{3}\right]$ on fivebrane world-volume, incorporating knots and links corresponds to introducing a $1 \mathrm{~d}$ defect ("impurity") which preserves the same supersymmetry as the background along $D^{2} \cong \mathbb{R}_{q}^{2}$ in our previous discussion, $c f$. Figure 1.

Alternatively, starting from the brane system (2.13), there are many possibilities [42] to accommodate knots inside $M_{3}$. We focus on those which give rise to line operators in $T\left[M_{3}\right] .{ }^{37}$ They correspond to codimension-four defects in the $6 \mathrm{~d}(2,0)$ theory, engineered by either M2or M5-branes (see e.g. [57] for a discussion in the context of 3d-3d correspondence). For

\footnotetext{
${ }^{37}$ The other options lead to a $3 \mathrm{~d}$ "space-time filling defect," deforming the theory to a new one $T\left[M_{3}, K\right]$.
} 
example, one can insert a stack of M2-branes in the following way:

$$
\begin{aligned}
& N \text { fivebranes: } \quad \mathbb{R} \times M_{3} \times D^{2} \\
& \cap \cap \\
& \text { space-time: } \quad \mathbb{R} \times T^{*} M_{3} \times T N \text {. } \\
& \cup \quad U \\
& \text { M2-branes: } \quad \mathbb{R} \times T^{*} K \times O
\end{aligned}
$$

We have chosen the line defect in $T\left[M_{3}\right]$ to sit at the tip $O \in D^{2}$ (also the Taub-NUT center) to preserve the $U(1)_{q}$ rotational symmetry. Naively, the way the stack of M2-branes ends on $N$ fivebranes is determined by a partition of the number of M2 branes into $N$. Such partitions are in one-to-one correspondence with Young tableaux with at most $N$ rows, which are in turn can be identified with irreducible representations of $U(N)$. In the case of $S U(N)$ we have to mod out configurations where M2 branes are equally distributed among $N$ fivebranes. This leads to Young tableaux with at most $N-1$ rows that label irreducible representations of $S U(N)$. In reality, mapping line operators in $3 \mathrm{~d} / 3 \mathrm{~d}$ correspondence and in the fivebrane setup is a delicate business and should be dealt with care.

If one wishes to use M5-branes to represent knots, then besides $\mathbb{R}_{\text {time }} \times K$ they would also occupy the two-dimensional co-normal bundle $N^{*} K$ of $K$ inside $T^{*} M_{3}$ and the cotangent space $\left.T^{*}\right|_{O}$ to $D^{2}$ at $O$ [15]. Both constructions, based on M2 and M5 branes, lead to Wilson loops - in fact, they are related by the Hanany-Witten type effect in M-theory or, in TQFT on $M_{3}$, by the Fourier transform from representation basis to holonomy basis.

Using this physical setup, we compute the new homological invariants $\mathcal{H}_{a}\left[M_{3} ; K, \mathcal{R}\right]$ for some simple examples of non-trivial knots and 3-manifolds. In particular, we verify that suitable variants of the Conjectures 2.1 and 2.2 hold in the presence of knots and links.

\subsection{Links in $M_{3}$ and line operators in $T\left[M_{3}\right]$}

Consider a knot $K$ in $M_{3}$ colored by a representation $\mathcal{R}$ of $G$. As described above, it corresponds to a certain half-BPS line operator $\Gamma_{K, \mathcal{R}}$ in $T\left[M_{3} ; G\right]$ :

$$
(K, \mathcal{R}) \longmapsto \Gamma_{K, \mathcal{R}} \in \mathcal{C} \text {. }
$$

where $\mathcal{C}$ is the category of BPS line operators in $\mathcal{N}=2 \operatorname{SCFT} T\left[M_{3}, G\right]$. The homological invariant for a combined system of a knot and a 3-manifold is then given by the BPS Hilbert space of $T\left[M_{3}\right]$ on the disk $D^{2}$ with $\Gamma_{K, \mathcal{R}}$ inserted at $O$, the center of the disk (see Figure 1):

$$
\mathcal{H}_{a}\left[M_{3} ; K, \mathcal{R}\right] \equiv \mathcal{H}_{T\left[M_{3}\right]}\left(D^{2}, \Gamma_{K, \mathcal{R}} ; a\right), \quad a \in\left(\text { Tor } H_{1}\left(M_{3}, \mathbb{Z}\right)\right)^{N} / S_{N}
$$

where $a$ denotes the choice of boundary condition, the same as in the case without a knot. It has the same gradings as before, namely the $q$-grading $i$ and the homological (R-charge) grading $j$ :

$$
\mathcal{H}_{a}\left[M_{3} ; K, \mathcal{R}\right]=\bigoplus_{i \in \Delta_{a}+\mathbb{Z}, j \in \mathbb{Z}} \mathcal{H}_{a}^{i, j}\left[M_{3} ; K, \mathcal{R}\right]
$$


And, as before, the graded Euler characteristic gives the partition function on $D^{2} \times S^{1}$ :

$$
\widehat{Z}_{a}\left(q ; \Gamma_{K, \mathcal{R}}\right) \equiv Z_{T\left[M_{3}\right]}\left(D^{2} \times S^{1}, \Gamma_{K, \mathcal{R}} ; a\right)=\sum_{i, j}(-1)^{j} q^{i} \mathcal{H}_{a}^{i, j}\left[M_{3} ; K, \mathcal{R}\right]
$$

now, in $3 \mathrm{~d} \mathcal{N}=2$ theory $T\left[M_{3}\right]$ with an extra $1 \mathrm{~d}$ "impurity" $\Gamma_{K, \mathcal{R}}$ supported on $O \times S^{1}$. The analogue of the Conjecture 2.1 is then the following:

Conjecture 4.1 The WRT invariant of $M_{3}$ with a knot $K$ colored by representation $\mathcal{R}$ can be decomposed into the following form:

$$
Z_{S U(2)_{k}}\left[M_{3} ; K, \mathcal{R}\right]=\left.(i \sqrt{2 k})^{b_{1}\left(M_{3}\right)-1} \sum_{\substack{a, b \in \\ \text { Tor } H_{1}\left(M_{3}, \mathbb{Z}\right) / \mathbb{Z}_{2}}} e^{2 \pi i k \ell k(a, a)} S_{a b} \widehat{Z}_{b}\left(q ; \Gamma_{K, \mathcal{R}}\right)\right|_{q \rightarrow e^{\frac{2 \pi i}{k}}}
$$

where

$$
\widehat{Z}_{b}\left(q ; \Gamma_{K, \mathcal{R}}\right) \in 2^{-c} q^{\Delta_{b}} \mathbb{Z}[[q]] \quad \Delta_{b} \in \mathbb{Q}, \quad c \in \mathbb{Z}_{+}
$$

convergent in $|q|<1$ and $S_{a b}$ is the same as in Conjecture 2.1

For certain 3-manifolds with an extra symmetry, preserved by the knot $K$, we have an extra grading whose value we denote by $\ell$ :

$$
\mathcal{H}_{a}\left[M_{3} ; K, \mathcal{R}\right]=\bigoplus_{i \in \Delta_{a}+\mathbb{Z}, j, \ell \in \mathbb{Z}} \mathcal{H}_{a}^{i, j ; \ell}\left[M_{3} ; K, \mathcal{R}\right]
$$

In practice, to compute these homological invariants, one needs a convenient ultra-violet (UV) description of the fivebrane setup (4.1) or, better yet, of the IR superconformal theory $T\left[M_{3}\right]$. In general, the brane system (4.1) and the $3 \mathrm{~d}$ theory $T\left[M_{3}\right]$ may admit many such UV descriptions, in various duality frames, all of which flow to the same IR physics and produce the same homological invariants (space of BPS states).

In a given UV gauge theory description of $T\left[M_{3}\right]$, the objects in the tensor category $\mathcal{C}$ of BPS line operators can be understood as vector spaces - the "Hilbert spaces of $\mathcal{N}=2$ quantum mechanics on the line, coupled to the bulk gauge theory". The most familiar line operators are Wilson operators $W_{\lambda}$, labeled by a representation $\lambda$ of the UV gauge group ${ }^{38}$ $\mathcal{G}$ (which, in general, has little to do with $G$ ). The corresponding object of the category $\mathcal{C}$ is, tautologically, the representation $\lambda$. The Wilson lines are expected to form a complete basis of line operators in the IR theory, in the sense that any line operator (not necessarily associate to a knot) should have the following decomposition:

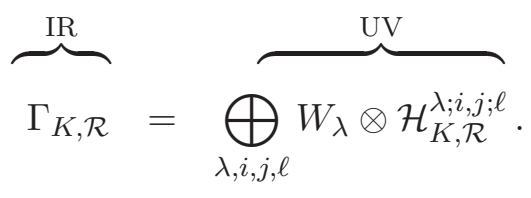

\footnotetext{
${ }^{38}$ In general, $\mathcal{G}$ is different from $G$. For a quiver theory, it may be given by a product $\times{ }_{i} G_{i}$ over the vertices.
} 
The reason is very simple - one can always decompose the "defect Hilbert space" into representations of $\mathcal{G}$, with the multiplicity space $\mathcal{H}_{\Gamma}^{\lambda}$ associated with the representation $\lambda$. The symmetry $\mathcal{G}$ plays the role of the flavor symmetry in QM on the line, which couples to $3 \mathrm{~d}$ theory $T\left[M_{3}\right]$ by gauging $\mathcal{G}$ in the bulk. If $\Gamma$ is a generic physical line operator ( $c f$. superposition of physical line operators), the corresponding multiplicity spaces $\mathcal{H}_{\Gamma}^{\lambda}$ are honest vector space that are at least doubly-graded, with the $(i, j)$-grading coming from $U(1)_{q} \times U(1)_{R}$ symmetry of the brane system (4.1). When the flavor symmetry $U(1)_{\beta}$ is a symmetry of both $T\left[M_{3}\right]$ and $\Gamma$, the third $\ell$-grading is also present.

As a trivial example, consider a line defect given by a quantum mechanics that is completely decoupled from the bulk. Then,

$$
\Gamma_{\mathrm{QM}}=W_{0} \otimes \mathcal{H}_{\mathrm{QM}}
$$

where $\mathcal{H}_{\mathrm{QM}}$ is the Hilbert space of the quantum mechanics and $W_{0}$ is the trivial Wilson loop. So, we see that (4.9) has a chance of being correct, even if there are infinitely many possibilities of engineering defects via $1 \mathrm{~d}-3 \mathrm{~d}$ coupled systems. In this sense, $\lambda$ is analogous to the label " $a$ " of the homological blocks, which reduces infinitely many possible boundary conditions to a finite set. In fact, this isn't just an analogy - it comes from the equivalence of two categories

$$
\mathcal{C} \cong \mathcal{C}_{B}
$$

Namely, a line operator can be viewed as the interface between two boundary conditions, and picking a "reference boundary condition" (e.g. the one giving $\widehat{Z}_{0}$ with zero brane charge) will identify the two categories. This equivalence is a special feature of $3 \mathrm{~d}$ quantum field theories, which associate categories to $S^{1}$, with the objects that can be interpreted either as line operators or as boundary conditions at spatial infinity.

At this point, $\lambda$ can still be an arbitrary representation of the UV gauge group $\mathcal{G}$, and one might naively think that $\mathcal{C}$ is

$$
\mathcal{C}_{\mathrm{UV}}=\operatorname{Rep}(\mathcal{G})
$$

given by the representation category of $\mathcal{G}$. But in light of the equivalence (4.11), we expect $\mathcal{C}$ to be much smaller, since $\mathcal{C}_{B}$ is generated by finitely many simple objects (boundary conditions that give homological blocks), as we have seen in all our examples.

Indeed, (4.12) is often redundant, due to presence of Chern-Simons couplings in $T\left[M_{3}\right]$. There is a set $\Lambda_{M_{3}}$ of "integrable representations" of $\mathcal{G}$ giving a complete basis of $W_{\lambda}$ in $T\left[M_{3} ; G\right]$, and every Wilson loop labeled by a non-integrable representation can be decomposed as

$$
W_{\nu}=\bigoplus_{\substack{\lambda \in \Lambda_{M_{3}} \\ i, j, \ell \in \mathbb{Z}}} W_{\lambda} \otimes \mathcal{H}_{\nu}^{\lambda ; i, j ; \ell} .
$$

All such relations generate an ideal (in the categorical sense) of $\mathcal{C}_{\mathrm{UV}}$, and $\mathcal{C}$ is given by the quotient

$$
\mathcal{C}=\mathcal{C}_{U V} / \mathcal{I}
$$


As a vector space, $\mathcal{C}$ is the same as $\Lambda_{M_{3}} \otimes \mathbb{C}$, but the algebra structure depends on $\mathcal{I}$ and is determined by the theory $T\left[M_{3}\right]$ in a non-trivial way. Both $\mathcal{C}_{\mathrm{UV}}$ and $\mathcal{I}$ depend on the duality frame (presentation of $M_{3}$ ), but it is expected that the quotient category $\mathcal{C}$ is a topological invariant of $M_{3}$. It describes line operators / boundary conditions in the IR theory, which is what usually meant by $T\left[M_{3}\right]$.

We expect, for arbitrary $M_{3}$, the independent Wilson loops in the theory $T\left[M_{3} ; G\right]$ are labeled by elements in ${ }^{39}$

$$
\Lambda_{M_{3}}=\operatorname{Tor} H_{1}\left(M_{3}, \Lambda_{\mathrm{rt}}\right) / W_{G},
$$

where $\Lambda_{\text {rt }}$ is the root lattice of $\mathfrak{g}$ and $W_{G}$ is the Weyl group (not to be confused with Wilson line $W_{\lambda}$ in UV description of $T\left[M_{3}\right]$ ). The argument for this statement parallels our discussion of boundary conditions given by M2-brane charges " $a$ ", which take values in exactly the same set. And the fundamental Wilson loops are expected to come from knots $K$ wrapping torsion 1-cycles, colored by a representation $\mathcal{R}$ of $G$ (i.e. $\left.\mathcal{R} \in \Lambda_{\text {char }}(G) / W_{G}\right)$.

If $G$ is abelian, then $\Lambda_{M_{3}}$ is a group and one can introduce an action of line operators on boundary conditions, which we call the shift action in what follows. This action is expected because Wilson line operators $W_{\lambda}, \lambda \in \Lambda_{M_{3}}$, can be engineered using M2-branes ${ }^{40}$ as in (4.1), and the label " $a$ " for homological blocks also measures M2-brane charges. In other words, a Wilson loop $W_{\lambda}$ relates two Hilbert spaces

$$
\mathcal{H}_{T\left[M_{3}\right]}\left(D^{2}, W_{\lambda} ; a\right) \sim \mathcal{H}_{T\left[M_{3}\right]}\left(D^{2}, W_{\lambda} ; a-\lambda\right) .
$$

One does not a priori know whether this is an isomorphism or not, or how the gradings on the two sides are related. However, in various examples below we observe that, at the level of homological blocks $\widehat{Z}_{a}(q)$, Wilson loop acts as a simple shift operator, even for $G=S U(2)$ if the Weyl group action is properly taken into account.

As before, our main tools for probing the new homological invariants are SUSY-protected quantities. In particular, using a UV descriptions of $T\left[M_{3}\right]$ and a half-BPS impurity $\Gamma_{K, \mathcal{R}}$, one can compute the partition function of $T\left[M_{3}\right]$ on $D^{2} \times S^{1}$ with $\Gamma_{K, \mathcal{R}}$ inserted along $O \times S^{1}$ as follows:

$$
\begin{aligned}
\widehat{Z}_{a}\left(q, t ; \Gamma_{K, \mathcal{R}}\right) \equiv & Z_{T\left[M_{3}\right]}\left(D^{2} \times S^{1}, \Gamma_{K, \mathcal{R}} ; a\right)= \\
& =\int \frac{d z}{2 \pi i z}\left[\text { same as without } \Gamma_{K, \mathcal{R}}\right] \sum_{\substack{\lambda \in \Lambda_{M_{3}} \\
i, j, \ell \in \mathbb{Z}}} \chi_{\lambda}(z) q^{i} t^{\ell}(-1)^{j} \operatorname{dim} \mathcal{H}_{K, \mathcal{R}}^{\lambda ; i, j, \ell}
\end{aligned}
$$

where $z$ stands for the gauge fugacities of the UV gauge group $\mathcal{G}$ and $\chi_{\lambda}$ is the character of the $\mathcal{G}$-representation $\lambda$.

\footnotetext{
${ }^{39}$ So far, we have ignored the difference between $G$ and the Langlands dual group $G^{\vee}$ because the quantities considered so far are not sensitive to the global structure of the group. However, the spectrum of Wilson loops is sensitive to this difference, and (4.15) is stated for the adjoint form $G=G_{\text {ad }}$. If $G$ has a center, one needs to add the representations of $G$ where the center acts non-trivially. Reader interested in this subject may consult [48] where this issue was extensively discussed in a similar brane system.

${ }^{40}$ i.e. $W_{\lambda}=\Gamma_{K, \mathcal{R}}$ when torsion homology class of $\mathcal{R}$-cable of $K$ is given by $\lambda \in \Lambda_{M_{3}}$
} 
Similarly, we can consider the $S^{2} \times S^{1}$ superconformal index with half-BPS impurities inserted at one (or both) poles of $S^{2}$. The UV localization formula for the index is modified as follows (when the impurity is at the north pole):

$$
\begin{aligned}
\mathcal{I}\left(q, t ; \Gamma_{K, \mathcal{R}}\right) & \equiv Z_{T\left[M_{3}\right]}\left(S^{2} \times S^{1} ; \Gamma_{K, \mathcal{R}}\right)= \\
= & \sum_{m} \int \frac{d z}{2 \pi i z}[\text { same as without } \Gamma] \sum_{\substack{\lambda \in \Lambda_{M_{3}} \\
i, j, \ell \in \mathbb{Z}}} \chi_{\lambda}\left(z q^{m / 2}\right) q^{i} t^{\ell}(-1)^{j} \operatorname{dim} \mathcal{H}_{K, \mathcal{R}}^{\lambda ; i, j ; \ell} .
\end{aligned}
$$

As we demonstrate in a variety of examples, it obeys the modified factorization formula

$$
\mathcal{I}\left(q, t ; \Gamma_{K, \mathcal{R}}\right)=\sum_{a}\left|\mathcal{W}_{a}\right| \widehat{Z}_{a}\left(q, t ; \Gamma_{K, \mathcal{R}}\right) \widehat{Z}_{a}\left(q^{-1}, t^{-1}\right)
$$

Note, all of the above relations depend only on the IR data, namely on $K$ and its color $\mathcal{R}$, not on the UV description of $\Gamma_{K, \mathcal{R}}$. In particular, note that $\lambda$ is always summed over in these relations, which goes back to the basic relation between IR line operators $\Gamma_{K, \mathcal{R}}$ ("impurities") and their description (4.9) in the UV theory via $W_{\lambda}$.

The reader should keep in mind that there are two non-trivial maps (dualities) involved in the study of knots. ${ }^{41}$ First, there is a rather non-trivial map (4.9) between line operators in the IR theory $T\left[M_{3}\right]$ and its UV description (or, rather, one of many UV descriptions). This UV/IR map has nothing to do with applications to low-dimensional topology and is a standard phenomenon in quantum field theory. In addition, there is a map between knots and links colored by $\mathcal{R}$ in a TQFT on $M_{3}$ and half-BPS line operators ("impurities") in the $3 \mathrm{~d} \mathcal{N}=2$ theory $T\left[M_{3}\right]$. This map is a chapter in the so-called $3 \mathrm{~d} / 3 \mathrm{~d}$ correspondence. The reason one should pay attention to both of these maps is that it is their composition which relates homological invariants of $K \subset M_{3}$ to concrete calculations in $T\left[M_{3} ; G\right]$,

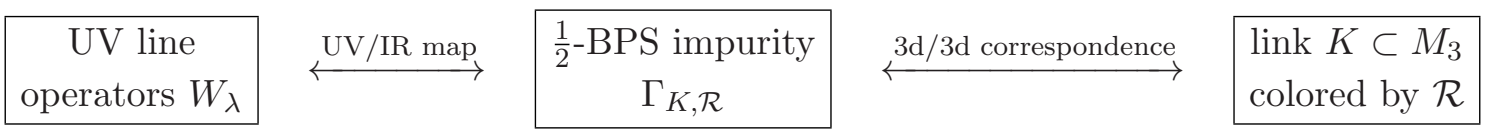

Because each of these maps can be rather non-trivial, one should not confuse the representation $\lambda$ that labels half-BPS line operators in the UV description with the "color" of $K$. The latter is denoted by $\mathcal{R}$ and, whenever non-trivial, leads to the $\mathcal{R}$-colored link homology $[58,59]$ in our setup (4.1).

So far, the discussion in this section is very general and, as such, may be somewhat abstract. We will now give some very concrete examples for $G=S U(2)$.

\subsection{Links in $S^{2} \times S^{1}$ and Rozansky's proposal}

For $M_{3}=S^{2} \times S^{1}$, the theory $T\left[M_{3}\right]$ is a particular case of (3.120) with $p=0$ and $g=0$, that is the theory of $3 \mathrm{~d} \mathcal{N}=2$ vector multiplet with gauge group $\mathcal{G}=G=S U(2)$. In this

\footnotetext{
${ }^{41}$ This aspect is a surprising exception to the rule that knot homology is easier than 3-manifold homology.
} 
case Tor $H_{1}\left(M_{3}, \mathbb{Z}\right)=0$ and the only independent Wilson loop in $T\left[M_{3}\right]$ is the trivial Wilson loop. Also, there is only one homological block $\widehat{Z}_{0}$. Therefore, for an arbitrary representation $\lambda$ of $S U(2)$, the decomposition (4.13) allows to write the Wilson loop $W_{\lambda}$ as (4.10):

$$
W_{\lambda}=W_{0} \otimes \mathcal{H}_{\lambda}^{0 ; i, j ; \ell}
$$

In other words, in this case we expect a factorization

$$
\mathcal{H}_{a}\left[S^{2} \times S^{1} ; K^{\lambda}, \mathcal{R}_{\lambda}\right]=\bigoplus_{i, j, \ell \in \mathbb{Z}} \mathcal{H}_{\lambda}^{0 ; i, j ; \ell} \otimes \mathcal{H}_{a}\left[S^{2} \times S^{1}\right]
$$

where the first factor on the right-hand side is the $S^{2} \times S^{1}$ analogue of the Khovanov-Rozansky homology of the corresponding link $K^{\lambda}$ colored by $\mathcal{R}_{\lambda}$. What is $K^{\lambda}$ ? And, what is $\mathcal{R}_{\lambda}$ ?

As we shall see below, already in this relatively simple case of $M_{3}=S^{1} \times S^{2}$ the map between colored knots and links in $M_{3}$ and the UV line operators $W_{\lambda}$ in $T\left[M_{3}\right]$ is rather non-trivial. Since Wilson loops in $T\left[M_{3}\right]$ come from M2-branes wrapping the $S^{1}$ circle of $S^{1} \times S^{2}$, we expect that the precise dictionary $W_{\lambda} \leftrightarrow\left(K^{\lambda}, \mathcal{R}_{\lambda}\right)$ maps $\lambda$ to a certain link in $M_{3}$ that winds around $S^{1}$, and the total winding number (counted with multiplicities given by $\mathcal{R}_{\lambda}$ ) is equal to $|\lambda|$. Regarding $M_{3}=S^{1} \times S^{2}$ as a special case of the Hopf fibration (namely, the trivial fibration), in the rest of this section we present evidence for the following identification $^{42}$

$$
W_{\lambda} \leftrightarrow \quad\left(K^{\lambda}, \mathcal{R}_{\lambda}\right)=\text { " } \lambda \text {-cable" of the unknot along the Hopf fiber }
$$

Specifically, $\lambda=\square^{\otimes 2 n}$ corresponds to a link $L_{2 n}$ made of $2 n$ copies of the unknot along the Hopf fiber, all colored by the fundamental representation of $S U(2)$. And $\left(K^{\lambda}, \mathcal{R}_{\lambda}\right)$ for general $\lambda$ is a linear combination of $L_{2 n}$ 's that replicates the decomposition of $\lambda$ into $\square^{\otimes 2 n}$ 's. Such decomposition is usually referred to as the "cabling formula" (see Appendix B).

Curiously, a similar problem was already considered by Rozansky from a purely mathematical point of view in [62]. Specifically, the Conjecture 6.10 in [62] gives a concrete description for the homological invariant of the link $L_{2 n}$. We shall verify that Rozansky's proposal $^{43}$ matches exactly with the quantum field theory computation if the generators $a_{i}$ and $\theta_{i}$ are assigned flavor charges $i$, that is

$$
\operatorname{deg}_{t} a_{i}=\operatorname{deg}_{t} \theta_{i}=i .
$$

\footnotetext{
${ }^{42}$ Note, in the unrefined / decategorified setting, $K^{\lambda}$ is simply the unknot colored by $\mathcal{R}_{\lambda}=\lambda$. Although this is no longer the case in homological world, there is still a close relation between "cabling" and "color" [60,61].

${ }^{43}$ The Conjecture 6.10 in [62] contains a typo in the description of the ideal $I_{\text {rel }}$. Namely,

$$
a_{i} p_{i}(\mathbf{x})=\theta_{i} p_{i}(\mathbf{x})=0
$$

should be replaced by

$$
a_{i} p_{n-i+1}(\mathbf{x})=\theta_{i} p_{n-i+1}(\mathbf{x})=0 .
$$


Using the decomposition of tensor products of $S U(2)$ representations, we can write the expectation value of $2 n$ Wilson loops in $T\left[M_{3}\right]$ as a sum over expectation values of Wilson loops labeled by irreducible representations. Only even representations with highest weight $2 l=0,2, \ldots, 2 n$ appear in this decomposition:

$$
\left\langle W_{\square \otimes 2 n}\right\rangle=\sum_{l=0}^{n}\left[\left(\begin{array}{c}
2 n \\
n-l
\end{array}\right)-\left(\begin{array}{c}
2 n \\
n-l-1
\end{array}\right)\right]\left\langle W_{2 l}\right\rangle .
$$

The expectation value of the Wilson loop $W_{2 l}$ in $T\left[M_{3}\right]$, which gives the graded Euler characteristic of $\mathcal{H}_{\lambda},{ }^{44}$

$$
\sum_{i, j, \ell}(-1)^{j} q^{i} t^{\ell} \operatorname{dim} \mathcal{H}_{\lambda}^{0 ; i, j, \ell} \equiv \chi_{q, t}\left(\mathcal{H}_{\lambda}\right)=\left\langle W_{2 l}\right\rangle \equiv \frac{\widehat{Z}_{0}\left(q, t ; W_{2 l}\right)}{\widehat{Z}_{0}(q, t)}
$$

can be computed using (4.17), with $T\left[S^{1} \times S^{2}, S U(2)\right]$ now being the $3 \mathrm{~d} \mathcal{N}=4$ super-YangMills theory. We obtain

$$
\left\langle W_{2 l}\right\rangle=\frac{(q t)^{l}(1 / t ; q)_{l}}{\left(q^{2} t ; q\right)_{l}},
$$

and the expectation values of $W_{2 l+1}$ all vanish.

On the other hand, as a vector space, the homological invariant $H^{\bullet}\left(S^{1} \times S^{2}, L_{2 n}\right)$ proposed in Conjecture 6.10 of [62] admits a similar decomposition into subspaces of pure $\mathbf{x}$-degree ${ }^{45}$

$$
H_{\text {Rozansky }}^{\bullet}\left(L_{2 n}\right)=\bigoplus_{l=0}^{n} V_{l} \otimes \mathbb{Q}\left[a_{1}, \theta_{1}, a_{2}, \theta_{2}, \ldots, a_{l}, \theta_{l}\right] .
$$

Here, $V_{l}$ is a quotient of the vector space spanned by monomials of $x_{1}, \ldots, x_{2 n}$, with degree $n-l$, by the relations

$$
x_{i}^{2}=0 \quad \text { and } \quad \sum_{i=1}^{2 n} x_{i}=0 .
$$

It is easy to check that ${ }^{46}$

$$
\operatorname{dim} V_{l}=\left(\begin{array}{c}
2 n \\
n-l
\end{array}\right)-\left(\begin{array}{c}
2 n \\
n-l-1
\end{array}\right)
$$

are exactly the coefficients in (4.26). In particular, $V_{j}$ with $j<0$ is empty, and the dimension of $V_{0}$ is given by the $n$-th Catalan number. Then

$$
\chi_{t}\left(H^{\bullet}\right)=\sum_{j=0}^{n} \operatorname{dim} V_{l} \cdot \mathfrak{q}^{2 n-2 j} \cdot \frac{\left(t ; t \mathfrak{q}^{-2}\right)_{j}}{\left(t \mathfrak{q}^{-4} ; t \mathfrak{q}^{-2}\right)_{j}},
$$

\footnotetext{
${ }^{44}$ In this normalization, we have

$$
\left\langle W_{0}\right\rangle=1,
$$

enabling us to directly extract the information about the space $\mathcal{H}_{\lambda}^{i, j ; \ell}$.

${ }^{45}$ This decomposition doesn't respect the ring structure as each subspace is not closed under multiplication.

${ }^{46}$ Basically, there are $\left(\begin{array}{c}2 n \\ n-l\end{array}\right)$ non-vanishing degree $(n-l)$ monomials, and they are subject to $\left(\begin{array}{c}2 n \\ n-l-1\end{array}\right)$ independent relations, obtained by multiplying $\sum_{i} x_{i}$ with degree $(n-l-1)$ monomials.
} 
where we have used the q-degree of the generators,

$$
\begin{aligned}
\operatorname{deg}_{\mathfrak{q}} x_{i} & =2, \\
\operatorname{deg}_{\mathfrak{q}} a_{i} & =-2 i-2, \\
\operatorname{deg}_{\mathfrak{q}} \theta_{i} & =-2 i+2 .
\end{aligned}
$$

If we shift the overall $\mathfrak{q}$-degree of $H^{\bullet}$ by $-2 n$ units to remove the $\mathfrak{q}^{2 n}$ factor in (4.33), what remains is in perfect agreement with the expectation value of Wilson loops in $3 \mathrm{~d}$ theory $T\left[M_{3}\right]:$

$$
\left\langle W_{2 l}\right\rangle=(q t)^{j} \cdot \frac{(1 / t ; q)_{j}}{\left(t q^{2} ; q\right)_{j}}=\mathfrak{q}^{-2 j} \cdot \frac{\left(t, t \mathfrak{q}^{-2}\right)_{j}}{\left(t \mathfrak{q}^{-4} ; t \mathfrak{q}^{-2}\right)_{j}}
$$

with a typical relation between $q$ and $\mathfrak{q}[59]$ :

$$
\mathfrak{q}^{2}=q t
$$

So, we have verified the conjecture by Rozansky at the level of equivariant Euler characteristics for this link $L_{2 n}$,

$$
\chi_{q, t}\left(\mathcal{H}_{\mathrm{BPS}}\right)=\chi_{q, t}\left(H_{\text {Rozansky }}\right)
$$

presenting some evidence for the isomorphism between $H_{\text {Rozansky }}^{\bullet}\left(S^{1} \times S^{2}, L_{2 n}\right)\{-2 n\}$ and $\mathcal{H}_{\lambda}:=\bigoplus_{i, j, \ell \in \mathbb{Z}} \mathcal{H}_{\lambda}^{0 ; i, j ; \ell}$ with $\lambda=\square^{\otimes 2 n}$.

As a side remark, positivity also features in the invariants $\widehat{Z}_{a}\left(M_{3} ; K_{\mathcal{R}}\right)$ of three manifolds with knots, in a way similar to the case without knot (3.87). For example, in our case of $M_{3}=S^{2} \times S^{1}$,

$$
\begin{aligned}
& -\widehat{Z}_{0}\left(q,-t ; W_{6}\right)=\left(2 t^{3}+2 t^{2}\right) q^{3}+\left(2 t^{4}+4 t^{3}+4 t^{2}+2 t\right) q^{4}+ \\
& \left(2 t^{5}+6 t^{4}+10 t^{3}+10 t^{2}+4 t\right) q^{5}+\left(2 t^{6}+8 t^{5}+16 t^{4}+22 t^{3}+18 t^{2}+8 t+2\right) q^{6}+ \\
& \left(2 t^{7}+8 t^{6}+20 t^{5}+36 t^{4}+44 t^{3}+34 t^{2}+14 t+2\right) q^{7}+O\left(q^{8}\right)
\end{aligned}
$$

has only positive coefficients.

\subsection{Links in $L(p, 1)$}

For $M_{3}=L(p, 1)$, or more generally $M_{3}=O(-p) \rightarrow \Sigma_{g}$, we have $\Lambda_{M_{3}}=\mathbb{Z}_{p} / \mathbb{Z}_{2}$ and the basic Wilson loops in $T\left[M_{3}\right]$ are given by $W_{\lambda}$ with $\lambda=0, \ldots, p \cdot{ }^{47}$ These correspond to the " $\lambda$-cablings" of the unknot (4.22) wrapping the Hopf fiber of $L(p, 1)$.

More generally, for plumbed $M_{3}$ (see section 3.4), if $\left(K^{\lambda}, \mathcal{R}_{\lambda}\right)$ is a $\lambda$-cabling of the unknot wrapping the "Hopf fiber" associated with the node $v$ of the plumbing graph, we have $\Gamma_{K, \lambda}=W_{\lambda_{v}}$, where $\lambda_{v}$ is the representation of $\mathcal{G}$ obtained from the representation $\lambda$ of $G$ via the "evaluation homomorphism" $e_{v}: \mathcal{G} \rightarrow G$ for the node $v$.

\footnotetext{
${ }^{47}$ Notice that we have added odd representations according to description in footnote 39.
} 


\subsubsection{Unrefined index of $T[L(p, 1)]$ with Wilson loops}

The unrefined superconformal index - with contribution of the Cartan component of the adjoint chiral removed, as in (3.40) — with the Wilson line inserted at the north pole reads:

$$
\begin{aligned}
\left\langle W_{\lambda}\right\rangle \equiv \mathcal{I}\left(q ; W_{\lambda}\right)= & \frac{1}{2} \sum_{m \in \mathbb{Z}} \int \frac{d z}{2 \pi i z} z^{p m} \cdot \chi_{\lambda}\left(q^{m / 2} z\right) \\
& \times\left(q^{2|m|}+q^{-2|m|}+4-2\left(q^{|m|}+q^{-|m|}\right)\left(z^{2}+\frac{1}{z^{2}}\right)+\left(z^{4}+\frac{1}{z^{4}}\right)\right) .
\end{aligned}
$$

Assuming $\lambda$ is irreducible, we identify it with its highest weight in $\Lambda_{\mathrm{wt}, \mathfrak{s u}(2)} / \mathbb{Z}_{2} \cong \mathbb{Z}_{\geq 0}$. Our convention is such that

$$
\chi_{\lambda}(z)=\frac{z^{\lambda+1}-z^{-\lambda-1}}{z-z^{-1}} .
$$

So, the insertion of an operator $W_{\lambda}$ with odd value of $\lambda$ automatically gives zero, because of the non-trivial action of the center $\mathbb{Z}_{2} \subset S U(2)$.

In the table below we present explicit results for $p=5$ up to $\lambda=38$ :

\begin{tabular}{|c|c||c|c||c|c||c|c||c|c|}
\hline$\lambda$ & $\left\langle W_{\lambda}\right\rangle$ & $\lambda$ & $\left\langle W_{\lambda}\right\rangle$ & $\lambda$ & $\left\langle W_{\lambda}\right\rangle$ & $\lambda$ & $\left\langle W_{\lambda}\right\rangle$ & $\lambda$ & $\left\langle W_{\lambda}\right\rangle$ \\
\hline 0 & 3 & 2 & -1 & 4 & 0 & 6 & $q^{-3}$ & 8 & $-q^{-3}-2 q^{-5}$ \\
\hline 10 & $2 q^{-5}+q^{-7}$ & 12 & $-q^{-7}$ & 14 & 0 & 16 & $q^{-16}$ & 18 & $-q^{-16}-2 q^{-20}$ \\
\hline 20 & $2 q^{-20}+q^{-24}$ & 22 & $-q^{-24}$ & 24 & 0 & 26 & $q^{-39}$ & 28 & $-q^{-39}-2 q^{-45}$ \\
\hline 30 & $2 q^{-45}+q^{-51}$ & 32 & $-q^{-51}$ & 34 & 0 & 36 & $q^{-72}$ & 38 & $-q^{-72}-2 q^{-80}$ \\
\hline
\end{tabular}

This table is almost periodic. This can be understood from the action of Wilson lines on the homological blocks. For the $D^{2} \times S^{1}$ partition function of $T\left[M_{3}\right]$ with $W_{\lambda}$ inserted at the center of the disk, $O \in D^{2}$, we have:

$$
\widehat{Z}_{a}\left(q ; W_{\lambda}\right)=\frac{1}{\left|\mathcal{W}_{a}\right|} \int \frac{d z}{2 \pi i z}\left(1-z^{ \pm 2}\right) \chi_{\lambda}(z) \sum_{n \in p \mathbb{Z}+a} q^{n^{2} / p} z^{2 n} .
$$

Wilson loops act in a very simple way on the homological blocks $\widehat{Z}_{a}(q)$. For $L(p, 1)$, we have

$$
\widehat{Z}_{0}=1, \quad \widehat{Z}_{1}=-q^{1 / p}
$$

with the rest of the blocks being zero. Let us also formally extend $\widehat{Z}_{a}$ for $a$ outside the range $\left\{0,1, \ldots,\left\lfloor\frac{p}{2}\right\rfloor\right\}$ by $\widehat{Z}_{a}=0$ and define a "shift operator"

$$
[j] \widehat{Z}_{a}(q):=q^{j(2 a-j) / p} \widehat{Z}_{a-j}(q), \quad a, j \in \mathbb{Z} .
$$

Then,

$$
\widehat{Z}_{a}\left(q ; W_{\lambda}\right)=\sum_{b \equiv \pm a}[\lambda / 2] \widehat{Z}_{b}(q), \quad a \in \mathbb{Z}_{p} / \mathbb{Z}_{2}
$$

This equation is not cyclic, and we took $a$ to live in $\mathbb{Z}_{\geq 0}$. Then, one can verify that

$$
\left\langle W_{\lambda}\right\rangle=\sum_{a \in \mathbb{Z}_{p} / \mathbb{Z}_{2}}\left|\mathcal{W}_{a}\right| \widehat{Z}_{a}\left(q ; W_{\lambda}\right) \widehat{Z}_{a}\left(q^{-1}\right)
$$


One can also deduce the action of $W_{\lambda}$ on $Z_{a}=\sum_{b} S_{a b} \widehat{Z}_{b}$ by applying the $S$-transform, but it won't be very illuminating because $Z_{a}\left(q ; W_{\lambda}\right)$ will be a linear combination of all $Z_{a}(q)$ 's. This difference between $\widehat{Z}_{a}$ and $Z_{a}$ again demonstrates the advantage of working with the homological blocks $\widehat{Z}_{a}$, rather than $Z_{a}(q)$.

\subsubsection{Refinement and categorification}

The refined version of (4.43) reads

$$
(q t ; q)_{\infty} \widehat{Z}_{a}\left(q, t ; W_{\lambda}\right)=\frac{1}{\left|\mathcal{W}_{b}\right|} \int \frac{d z}{2 \pi i z} \frac{\left(z^{2} ; q\right)_{\infty}\left(z^{-2} ; q\right)_{\infty}}{\left(z^{2} t q ; q\right)_{\infty}\left(z^{-2} t q ; q\right)_{\infty}} \chi_{\lambda}(z) \sum_{n \in p \mathbb{Z}+b} q^{n^{2} / p} z^{2 n}
$$

One can show that it has the positivity property (3.87) and, therefore, can be interpreted as the Poincaré polynomial of the homological invariant that categorifies $S U(2)$ WRT invariants of $M_{3}=L(p, 1)$ with knots wrapping the Hopf fiber.

For example, consider $M_{3}=L(5,1)$. With our choice of $G=S U(2)$, it has three abelian flat connections and, correspondingly, three homological blocks, all tabulated in [26, sec.6]. Now, we can see that incorporating knots does not spoil the positivity of (4.48) and its interpretation as the Poincaré polynomial. Yet, the presence of the knot changes all homological blocks in a non-trivial way, mixing homological invariants of the knot $K$ and the ambient 3-manifold $M_{3}$ in one combined entity, e.g. for $M_{3}=L(5,1)$ and $\lambda=2$ :

$$
\begin{gathered}
-(-q t ; q)_{\infty} \widehat{Z}_{0}\left(q,-t ; W_{2}\right)=(t+1) q+\left(t^{2}+2 t+1\right) q^{2}+\left(t^{3}+3 t^{2}+4 t+2\right) q^{3}+ \\
\left(t^{4}+4 t^{3}+8 t^{2}+8 t+3\right) q^{4}+\left(t^{5}+4 t^{4}+10 t^{3}+16 t^{2}+14 t+5\right) q^{5}+ \\
\left(t^{6}+4 t^{5}+11 t^{4}+23 t^{3}+31 t^{2}+23 t+7\right) q^{6}+ \\
\left(t^{7}+4 t^{6}+12 t^{5}+28 t^{4}+47 t^{3}+54 t^{2}+37 t+11\right) q^{7}+O\left(q^{8}\right), \quad(4.49) \\
(-q t ; q)_{\infty} \widehat{Z}_{1}\left(q,-t ; W_{2}\right)=\sqrt[5]{q}+(t+1) q^{6 / 5}+\left(2 t^{2}+4 t+2\right) q^{11 / 5}+\left(2 t^{3}+6 t^{2}+8 t+4\right) q^{16 / 5}+ \\
\left(2 t^{4}+7 t^{3}+14 t^{2}+15 t+6\right) q^{21 / 5}+\left(2 t^{5}+8 t^{4}+19 t^{3}+29 t^{2}+25 t+9\right) q^{26 / 5}+ \\
\left(2 t^{6}+8 t^{5}+22 t^{4}+43 t^{3}+55 t^{2}+41 t+13\right) q^{31 / 5}+O\left(q^{36 / 5}\right), \quad(4.50)
\end{gathered}
$$

and

$$
\begin{gathered}
-(-q t ; q)_{\infty} \widehat{Z}_{2}\left(q,-t ; W_{2}\right)=q^{4 / 5}+(t+1) q^{9 / 5}+\left(t^{2}+2 t+1\right) q^{14 / 5}+\left(2 t^{3}+4 t^{2}+4 t+2\right) q^{19 / 5}+ \\
\left(2 t^{4}+5 t^{3}+8 t^{2}+8 t+3\right) q^{24 / 5}+\left(2 t^{5}+5 t^{4}+11 t^{3}+17 t^{2}+13 t+4\right) q^{29 / 5}+ \\
\left(2 t^{6}+6 t^{5}+14 t^{4}+25 t^{3}+29 t^{2}+21 t+7\right) q^{34 / 5}+O\left(q^{36 / 5}\right) \cdot(4.51)
\end{gathered}
$$

Note that, when $p=1$, we have $L(p, 1) \cong S^{3}$ and the unknot wrapping the Hopf fiber becomes the familiar unknot in $S^{3}$, yet with a nontrivial framing equal to 1 . Since $H_{1}\left(S^{3}, \mathbb{Z}\right)=$ 0 , as in section 4.2 , we expect factorization in this case:

$$
W_{\lambda}=W_{0} \otimes \mathcal{H}_{\lambda}^{0 ; i, j ; \ell}
$$




$$
\mathcal{H}_{a}\left[S^{3} ; K^{\lambda}, \mathcal{R}_{\lambda}\right]=\bigoplus_{i, j, \ell \in \mathbb{Z}} \mathcal{H}_{\lambda}^{0 ; i, j ; \ell} \otimes \mathcal{H}_{a}\left[S^{3}\right]
$$

Moreover, as in section 4.2 , we expect that $\left(K^{\lambda}, \mathcal{R}_{\lambda}\right)$ is the cabling of the unknot determined by $\lambda$. In appendix $B$, we present evidence for this by comparing it with the ordinary Khovanov homology of the cabling of the unknot, $c f$. [60].

\subsection{Comparison with refined CS}

In this section, we wish to compare the categorification of WRT invariants of knots in 3manifolds considered in the previous section with analogous calculations using the tools of refined CS theory and DAHA $[19,51]$. To the best of our knowledge, such calculations (that involve both knots and 3-manifolds) were not done in the literature on refined Chern-Simons theory.

Let us consider the unknot $K$ wrapping the Hopf fiber in $M_{3}=L(p, 1)$ and colored by an irreducible representation $\mathcal{R}=\lambda$. Note, that now the representation $\lambda$ (whose weight we denote by the same letter) does represent the "color" of $K \subset M_{3}$. The partition function of the refined CS theory with such insertion can be expressed through $S$ and $T$ matrices (listed in section 3.2.6) as follows:

$$
\begin{aligned}
Z_{S U(2)}^{\text {ref. CS}} & {[L(p, 1) ; K, \lambda]=\sum_{n=1}^{k-1} g_{n}^{-1} S_{1 n} S_{n, \lambda+1}\left(T_{n n}\right)^{-p}=} \\
& =\sum_{n=1}^{k-1} q^{-\frac{p n^{2}}{4}-\frac{p n \beta}{2}-\frac{p \beta^{2}}{4}} \prod_{j=0}^{\beta}\left(q^{-\frac{n+j+\beta}{2}}-q^{\frac{n+j+\beta}{2}}\right)\left(q^{-\frac{n-j+\beta}{2}}-q^{\frac{n-j+\beta}{2}}\right) M_{\lambda}\left(q^{\beta+n}\right) .
\end{aligned}
$$

where $M_{\lambda}(x)$ is the $S U(2)$ Macdonald polynomial, whose coefficients are rational functions of $q$ and $t=q^{\beta}$. By repeating the manipulations of section 3.2.6, we obtain (up to the same simple overall factor):

$$
Z_{S U(2)}^{\text {ref. CS }}[L(p, 1) ; K, \lambda]=\sum_{a, b \in \mathbb{Z}_{p} / \mathbb{Z}_{2}} e^{2 \pi i(k+2 \beta) \operatorname{CS}(a)} \cdot S_{a b} \cdot \frac{1}{\left|\mathcal{W}_{b}\right|} \widehat{Z}_{b}^{(\text {ref.CS; } \beta)}(q ; K, \lambda)
$$

with

$$
\begin{aligned}
\widehat{Z}_{a}^{\text {(ref.CS; } \beta)}(q ; K, \lambda)=\int \frac{d z}{2 \pi i z} & \left(z^{ \pm 2} ; q\right)_{\beta+1} M_{\lambda}\left(z^{2}\right) \sum_{n \in p \mathbb{Z}+a} q^{n^{2} / p} z^{2 n}= \\
& =\left.\int \frac{d z}{2 \pi i z}\left(\frac{\left(z^{ \pm 2} ; q\right)_{\infty}}{\left(z^{ \pm 2} q t ; q\right)_{\infty}}\right) M_{\lambda}\left(z^{2}\right) \sum_{n \in p \mathbb{Z}+a} q^{n^{2} / p} z^{2 n}\right|_{t=q^{\beta}}
\end{aligned}
$$

For $\lambda=1$, the Macdonald polynomial coincides with the Schur polynomial, $M_{1}\left(z^{2}\right)=\chi_{1}(z)$, and the expression above agrees with the $D^{2} \times S^{1}$ partition function with a Wilson line insertion (4.48). However, in order to find an agreement for more general $\lambda$, we need to make 
a replacement $\chi_{\lambda}(z) \rightarrow M_{\lambda}\left(z^{2}\right)$ in (4.48). At the catogorified level, this means that (4.22) needs to be replaced by

$$
W_{\lambda} \rightsquigarrow \bigoplus_{\mu \leq \lambda} W_{\mu} \otimes \mathcal{H}_{\lambda}^{\mu}
$$

where $\mathcal{H}_{\lambda}^{\mu}$ are appropriate categorifications of the coefificients $C_{\lambda}^{\mu}(q, t)$ in the decomposition $M_{\lambda}\left(z^{2}\right)=\sum_{\mu \leq \lambda} C_{\lambda}^{\mu}(q, t) \chi_{\mu}(z)$. This suggests that the insertion of the Macdonald polynomial in the partition function of $3 \mathrm{~d} \mathcal{N}=2$ theory $T\left[M_{3}\right]$ corresponds to a $\lambda$-colored unknot wrapping the Hopf fiber of $M_{3}, c f$. (4.22):

$$
M_{\lambda}\left(z^{2}\right) \leftrightarrow K=\text { unknot along the Hopf fiber colored by } \mathcal{R}=\lambda
$$

It would be interesting to test this against many examples of $\lambda$-colored $\operatorname{sl}(N)$ homology for various knots and links $[42,51,58,59,63-65]$.

Note, that the coefficients in fusion rules of Macdonald polynomials are non-trivial rational functions of $q, t$. Therefore, the dependence on $\lambda$ in (4.56) does not agrees with the cabling formula, unlike the case of Wilson line insertions. On the other hand, the dependence on framing in the refined CS is simple and is given by the corresponding power of the matrix element $T_{\lambda+1, \lambda+1}$. In particular, when $p=1$ the result of (4.56) is

$$
\frac{\widehat{Z}_{0}^{(\text {ref.CS} ; \beta)}(q ; K, \lambda)}{\widehat{Z}_{0}^{(\text {ref.CS } ; \beta)}(q)}=T_{\lambda+1, \lambda+1} M_{\lambda}(q t) .
$$

Note that, for $\lambda>1$, it is a nontrivial rational function of $q$ and $t$, which means that the underlying doubly graded vector space is infinite dimensional, unlike the finite dimensional vector spaces produced by Wilson line insertions (see Appendix B).

\section{Acknowledgments}

We would like to thank J. E. Andersen, M. Aganagic, F. Benini, C. Cordova, A. Gadde, E. Gorsky, K. Hori, H. Kim, S. Nawata, M. Romo, S. Shakirov, L. Rozansky and K. Ye for useful comments and discussions. The work of S.G. and D.P. is supported in part by the U.S. Department of Energy, Office of Science, Office of High Energy Physics, under Award Number DE-SC0011632. In addition, the work of D.P. is supported by the center of excellence grant "Center for Quantum Geometry of Moduli Space" from the Danish National Research Foundation (DNRF95). P.P. gratefully acknowledges the support from Marvin L. Goldberger Fellowship and the DOE Grant DE-SC0009988. The research of C.V. is supported in part by NSF grant PHY-1067976. This work was performed in part (by P.P.) at Aspen Center for Physics which is supported by National Science Foundation grant PHY-1066293. The authors would like to thank Simons Center for Geometry and Physics and the organisers of the Simons Summer Workshop 2016, where the work on the project has begun, for generous hospitality. 


\section{A. $\mathbb{Z}[[q]]$-valued invariant for negative definite plumbed 3-manifolds}

We are interested in obtaining a certain analytic continuation of $s u(2)_{k}$ WRT invariant $[66,67]$ of a plumbed 3-manidfold $M_{3}(\Gamma),{ }^{48}$ where $\Gamma$ is a tree. Let us first consider the case of positive integer level, with $k \in \mathbb{Z}_{+}$. The colored Jones polynomial of link $\mathcal{L}(\Gamma)$ is explicitly known $(\text { see } e . g .[67])^{49}$ :

$$
\begin{gathered}
J[\mathcal{L}(\Gamma)]_{n_{1}, \ldots, n_{L}}=\frac{2 i}{\mathbb{q}^{1 / 2}-\mathbb{q}^{-1 / 2}} \prod_{v \in \text { Vertices } \cong\{1, \ldots, L\}} \mathbb{q}^{\frac{a_{v}\left(n_{v}^{2}-1\right)}{4}}\left(\frac{2 i}{\mathbb{q}^{n_{v} / 2}-\mathbb{q}^{-n_{v} / 2}}\right)^{\operatorname{deg}(v)-1} \times \\
\prod_{\left(v_{1}, v_{2}\right) \in \text { Edges }} \frac{\mathbb{q}^{n_{v_{1}} n_{v_{2}} / 2}-\mathbb{q}^{-n_{v_{1}} n_{v_{2} / 2}}}{2 i}
\end{gathered}
$$

where $\mathbb{q}=e^{2 \pi i / k}{ }^{50}$ For the purposes of computing the WRT invariant of 3-manifold obtained by Dehn surgery on a link $\mathcal{L}(\Gamma)$, it is useful to consider the following quantity:

$$
F[\mathcal{L}(\Gamma)] \equiv \sum_{n \in\{1, \ldots, k-1\}^{L}} J[\mathcal{L}(\Gamma)]_{n_{1}, \ldots, n_{L}} \prod_{v=1}^{L} \frac{\mathbb{q}^{n_{v} / 2}-\mathbb{q}^{-n_{v} / 2}}{\mathbb{q}^{1 / 2}-\mathbb{q}^{-1 / 2}}
$$

Then the WRT invariant of $M_{3}(\Gamma)$ is given by $[66]^{51}$

$$
\tau\left[M_{3}(\Gamma)\right]=\frac{F[\mathcal{L}(\Gamma)]}{F[\mathcal{L}(+1 \bullet)]^{b_{+}} F[\mathcal{L}(-1 \bullet)]^{b_{-}}}
$$

where $b_{ \pm}$are the number of positive/negative eigenvalues of the linking matrix $M$ while $\pm 1 \bullet$ denotes a plumbing graph with one vertex corresponding to an unknot with \pm 1 framing. The $S U(2)_{k}$ CS partition function, in the usual physical normalization, with

$$
Z_{S U(2)_{k}}\left[S^{2} \times S^{1}\right]=1,
$$

differs from (A.5) by a simple factor:

$$
Z_{S U(2)_{k}}\left[M_{3}(\Gamma)\right]=\sqrt{\frac{2}{k}} \sin \frac{\pi}{k} \cdot \tau\left[M_{3}(\Gamma)\right]
$$

\footnotetext{
${ }^{48}$ Everything below in principle can be generalized to $s u(N)$ and $u(N)$.

${ }^{49}$ We use the normalization in which the colored Jones polynomial of unlink with $L$ components with canonical framing is given by

$$
J[\mathrm{Unlink}]_{n_{1}, \ldots, n_{L}}=\prod_{v=1}^{L} \frac{\mathbb{q}^{n_{v} / 2}-\mathbb{q}^{-n_{v} / 2}}{\mathbb{q}^{1 / 2}-\mathbb{q}^{-1 / 2}} .
$$

${ }^{50}$ In this appendix, we use $q$ to denote a $k$-th $\left(k \in \mathbb{Z}_{+}\right)$root of unity $e^{\frac{2 \pi i}{k}}$ and reserve $q$ to denote the corresponding continuous variable which will appear in analytic continuation.

${ }^{51}$ Normalized such that

$$
\tau\left[S^{3}\right]=1
$$


The following Gauss sum reciprocity formula will be very useful for us (see e.g. $[68,69]$ ):

$$
\begin{aligned}
\sum_{n \in \mathbb{Z}^{L} / 2 k \mathbb{Z}^{L}} \exp \left(\frac{\pi i}{2 k}(n, M n)+\frac{\pi i}{k}(\ell, n)\right)= \\
\qquad \frac{e^{\frac{\pi i \sigma}{4}}(2 k)^{L / 2}}{|\operatorname{det} M|^{1 / 2}} \sum_{a \in \mathbb{Z}^{L} / M \mathbb{Z}^{L}} \exp \left(-2 \pi i k\left(a+\frac{\ell}{2 k}, M^{-1}\left(a+\frac{\ell}{2 k}\right)\right)\right)
\end{aligned}
$$

where $\ell \in \mathbb{Z}^{L},(\cdot, \cdot)$ is the standard pairing on $\mathbb{Z}^{L}$ and $\sigma=b_{+}-b_{-}$is the signature of the linking matrix $M$. In particular, from this formula it follows that

$$
F[\mathcal{L}( \pm 1 \bullet)]=\sum_{n=1}^{k-1} \mathbb{q}^{ \pm \frac{n^{2}-1}{4}}\left(\frac{\mathbb{q}^{n / 2}-\mathbb{q}^{-n / 2}}{\mathbb{q}^{1 / 2}-\mathbb{q}^{-1 / 2}}\right)^{2}=\frac{(2 k)^{1 / 2} e^{ \pm \frac{\pi i}{4}} \mathfrak{q}^{\mp \frac{3}{4}}}{\mathbb{q}^{1 / 2}-\mathbb{q}^{-1 / 2}}
$$

Therefore

$$
\begin{gathered}
\tau\left[M_{3}(\Gamma)\right]=\frac{e^{-\frac{\pi i \sigma}{4}} \mathbb{q}^{\frac{3 \sigma}{4}}}{2(2 k)^{L / 2}\left(\mathbb{q}^{1 / 2}-\mathbb{q}^{-1 / 2}\right)} \times \\
\sum_{n \in \mathbb{Z}^{L} / 2 k \mathbb{Z}^{L}} \prod_{v \in \text { Vertices }} \mathbb{q}^{\frac{a_{v}\left(n_{v}^{2}-1\right)}{4}}\left(\frac{1}{\mathbb{q}^{n_{v} / 2}-\mathbb{q}^{-n_{v} / 2}}\right)^{\operatorname{deg}(v)-2} \times \\
\prod_{\left(v^{\prime}, v^{\prime \prime}\right) \in \text { Edges }} \frac{\mathbb{q}^{n_{v^{\prime}} n_{v^{\prime \prime}} / 2}-\mathbb{q}^{-n_{v^{\prime}} n_{v^{\prime \prime}} / 2}}{2}
\end{gathered}
$$

where we used invariance of the summand under $n_{v} \leftrightarrow-n_{v}$ and the fact that $L-\mid$ Edges $\mid=1$. The prime' in the sum means that the singular values $n_{v}=0, k$ are omitted.

Consider the following factor in the expression above:

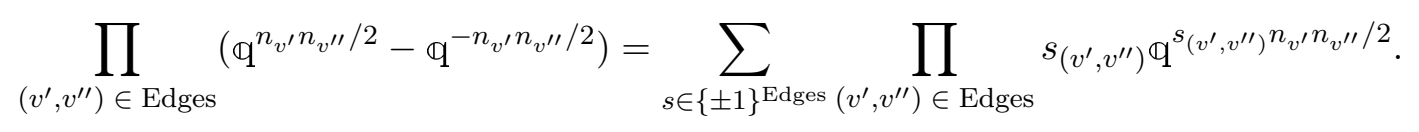

If one picks a vertex $v$ and makes a change $n_{v} \rightarrow-n_{v}$, a term in the sum with a given configuration of signs associated to edges (that is $s \in\{ \pm 1\}^{\text {Edges }}$ ) will transform into a term with a different configuration times $(-1)^{\operatorname{deg} v}$. Using the fact that the graph $\Gamma$ is a tree, by a sequence of such transforms, any configuration of signs can be brought to the configuration with all signs +1 . Therefore (A.10) can be rewritten as follows:

$$
\begin{aligned}
\tau\left[M_{3}(\Gamma)\right]=\frac{e^{-\frac{\pi i \sigma}{4}} \mathbb{q}^{\frac{3 \sigma-\sum_{v} a_{v}}{4}}}{2(2 k)^{L / 2}\left(\mathbb{q}^{1 / 2}-\mathbb{q}^{-1 / 2}\right)} \times \\
\sum_{n \in \mathbb{Z}^{L} / 2 k \mathbb{Z}^{L}} \mathbb{q}^{\frac{(n, M n)}{4}} \prod_{v \in \text { Vertices }}\left(\frac{1}{\mathbb{q}^{n_{v} / 2}-\mathbb{q}^{-n_{v} / 2}}\right)^{\operatorname{deg}(v)-2} .
\end{aligned}
$$


Before we can apply (A.8) to (A.12) we need to explicitly regularize the sum. In order to do this, let us introduce the following auxiliary quantities:

$$
\begin{gathered}
\Delta_{v} \in \mathbb{Z}_{+}: \Delta_{v}=\operatorname{deg} v-1 \bmod 2, \quad \forall v \in \text { Vertices, } \\
\omega \in \mathbb{C}: \quad 0<|\omega|<1 .
\end{gathered}
$$

Then

$$
\begin{gathered}
\sum_{n \in \mathbb{Z}^{L} / 2 k \mathbb{Z}^{L}} \mathbb{q}^{\frac{(n, M n)}{4}} \prod_{v \in \text { Vertices }}\left(\frac{1}{\mathbb{q}^{n_{v} / 2}-\mathbb{q}^{-n_{v} / 2}}\right)^{\operatorname{deg}(v)-2}= \\
\left.\lim _{\omega \rightarrow 1} \frac{1}{2^{L}} \sum_{n \in \mathbb{Z}^{L} / 2 k \mathbb{Z}^{L}} \mathbb{q}^{\frac{(n, M n)}{4}} F_{\omega}\left(x_{1}, \ldots, x_{L}\right)\right|_{x_{v}=\mathbb{q}^{n_{v} / 2}}
\end{gathered}
$$

where

$$
\begin{aligned}
& F_{\omega}\left(x_{1}, \ldots, x_{L}\right)= \\
& \prod_{v \in \text { Vertices }}\left(x_{v}-1 / x_{v}\right)^{\Delta_{v}}\left\{\left(\frac{1}{x_{v}-\omega / x_{v}}\right)^{\operatorname{deg}(v)-2+\Delta_{v}}+\left(\frac{1}{\omega x_{v}-1 / x_{v}}\right)^{\operatorname{deg}(v)-2+\Delta_{v}}\right\} \\
& \stackrel{\omega \approx 0}{=} \sum_{m \geq 0} \sum_{\ell \in \mathcal{I}_{m}} N_{m, \ell} \prod_{v} x_{v}^{\ell_{v}} \cdot \omega^{m} \in \mathbb{Z}\left[x_{1}^{ \pm 1}, \ldots, x_{L}^{ \pm 1}\right][[\omega]]
\end{aligned}
$$

with $\mathcal{I}_{m}$ being a finite set of elements from $\mathbb{Z}^{L}$. If, after expansion in $\omega$ one collects coefficients in front of powers of $x$, the expression has the following form:

$$
F_{\omega}\left(x_{1}, \ldots, x_{L}\right) \stackrel{\text { Formally }}{=} \sum_{\ell \in 2 \mathbb{Z}^{L}+\delta} F_{\omega}^{\ell} \prod_{v} x_{v}^{\ell_{v}} \quad \in \mathbb{Z}[\omega]\left[\left[x_{1}^{ \pm 1}, \ldots, x_{1}^{ \pm L}\right]\right]
$$

where

$$
F_{\omega}^{\ell}=\sum_{m: \ell \in \mathcal{I}_{m}} N_{m, \ell} \omega^{m} \in \mathbb{Z}[\omega]
$$

and

$$
\delta \in \mathbb{Z}^{L} / 2 \mathbb{Z}^{L}, \quad \delta_{v} \equiv \operatorname{deg} v \quad(\bmod 2) .
$$

Note that $F_{1}^{\ell}=\sum_{m} N_{m, \ell} \in \mathbb{Z}$ does not depend on the choice of $\Delta \in \mathbb{Z}^{L}$ in (A.13). Formally,

$$
\begin{aligned}
F_{1}\left(x_{1}, \ldots, x_{L}\right) & =\sum_{\ell \in 2 \mathbb{Z}^{L}+\delta} F_{1}^{\ell} \prod_{v} x_{v}^{\ell v}= \\
& \prod_{v \in \text { Vertices }}\left\{\begin{array}{c}
\text { Expansion } \\
\text { at } x \rightarrow 0
\end{array}\right.
\end{aligned}
$$


Now let us assume that the quadratic form $M: \mathbb{Z}^{L} \times \mathbb{Z}^{L} \rightarrow \mathbb{Z}$ is negative definite, ${ }^{52}$ i.e. $\sigma=$ $-L)$. Then we can define the following $q$-series, convergent for $|q|<1$ :

$$
\widehat{Z}_{b}(q) \stackrel{\text { Def }}{=} 2^{-L} q^{-\frac{3 L+\sum_{v} a_{v}}{4}} \sum_{\ell \in 2 M \mathbb{Z}^{L}+b} F_{1}^{\ell} q^{-\frac{\left(\ell, M^{-1} \ell\right)}{4}} \in 2^{-c} q^{\Delta_{b}} \mathbb{Z}[[q]]
$$

where

$$
\begin{gathered}
c \in \mathbb{Z}_{+}, \quad c \leq L, \\
b \in\left(2 \mathbb{Z}^{L}+\delta\right) / 2 M \mathbb{Z}^{L} / \mathbb{Z}_{2} \cong(2 \text { Coker } M+\delta) / \mathbb{Z}_{2} \stackrel{\text { Set }}{\cong} H_{1}\left(M_{3}, \mathbb{Z}\right) / \mathbb{Z}_{2}, \\
\Delta_{b}=-\frac{3 L+\sum_{v} a_{v}}{4}-\max _{\ell \in 2 M \mathbb{Z}^{L}+b} \frac{\left(\ell, M^{-1} \ell\right)}{4} \in \mathbb{Q}
\end{gathered}
$$

where $\mathbb{Z}_{2}$ acts as follows:

$$
b \rightarrow-b
$$

which is a symmetry of (A.21).

Using relation (A.15) and applying Gauss reciprocity formula (A.8) we arrive at the following expression for the WRT invariant:

$$
\begin{aligned}
& \tau_{k}\left[M_{3}(\Gamma)\right]=\left.\frac{e^{-\frac{\pi i \sigma}{4}} \mathbb{q}^{-\frac{3 L+\sum_{v} a_{v}}{4}}}{2(2 k)^{L / 2}\left(\mathbb{q}^{1 / 2}-\mathbb{q}^{-1 / 2}\right)} \lim _{\omega \rightarrow 1} \sum_{n \in \mathbb{Z}^{L} / 2 k \mathbb{Z}^{L}} \mathbb{q}^{\frac{(n, M n)}{4}} F_{\omega}\left(x_{1}, \ldots, x_{L}\right)\right|_{x_{v}=\mathbb{q}^{n_{v} / 2}}=
\end{aligned}
$$

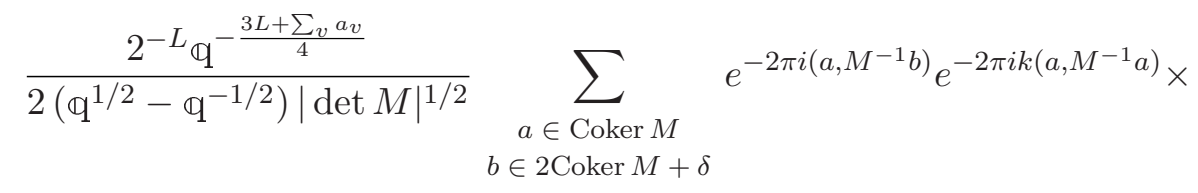

$$
\begin{aligned}
& \lim _{\omega \rightarrow 1} \sum_{\ell \in 2 M \mathbb{Z}^{L}+b} F_{\omega}^{\ell} \mathbb{q}^{-\frac{\left(\ell, M^{-1} \ell\right)}{4}}
\end{aligned}
$$

Now, assume that the $\operatorname{limit} \lim _{q \rightarrow \mathbb{q}} \widehat{Z}_{b}(q)$, where $\widehat{Z}_{b}(q)$ is defined in (A.21) and $q$ approaches $k$-th primitive root of unity from inside of the unit disc $|q|<1$, exists and moreover,

$$
\lim _{\omega \rightarrow 1} \sum_{\ell \in 2 M \mathbb{Z}^{L}+b} F_{\omega}^{\ell} \mathbb{q}^{-\frac{\left(\ell, M^{-1} \ell\right)}{4}}=\lim _{q \rightarrow \mathbb{q}} \sum_{\ell \in 2 M \mathbb{Z}^{L}+b} F_{1}^{\ell} q^{-\frac{\left(\ell, M^{-1} \ell\right)}{4}} .
$$

Then

$$
\tau_{k}\left[M_{3}(\Gamma)\right]=\frac{1}{2\left(\mathbb{q}^{1 / 2}-\mathbb{q}^{-1 / 2}\right)|\operatorname{det} M|^{1 / 2}} \times
$$

\footnotetext{
${ }^{52}$ In principle, this condition can be relaxed. It is only necessary that $M$ is negative on a certain subspace of $\mathbb{Z}^{L}$.
} 
We do not provide a proof of (A.27), and therefore (A.28), but believe it should be similar to the proof of the similar statement in [70]. However, even without the relation (A.28), the formula (A.21) provides an invariant of negative-definite plumbed 3-manifolds valued in $q$ series with integer coefficients. As was already mentioned before, the resulting $q$-series do not depend on the auxiliary choices of $\Delta_{v}$ in (A.16). One can show that they are also invariant under the action of Kirby moves (see Figure 5) acting on $\Gamma$, and therefore indeed depend only on homeomorphism class of $M_{3}(\Gamma)$.

The formula (A.21) for the homological blocks $\widehat{Z}_{a}$ can also be rewritten as a contour integral:

$$
\widehat{Z}_{b}(q)=q^{-\frac{3 L+\sum_{v} a_{v}}{4}} \cdot \text { v.p. } \int_{\left|z_{v}\right|=1} \prod_{v \in \text { Vertices }} \frac{d z_{v}}{2 \pi i z_{v}}\left(z_{v}-1 / z_{v}\right)^{2-\operatorname{deg}(v)} \cdot \Theta_{b}^{-M}(z)
$$

where $\Theta_{b}^{-M}(x)$ is the theta function of the lattice corresponding to minus the linking form $M$ :

$$
\Theta_{b}^{-M}(x)=\sum_{\ell \in 2 M \mathbb{Z}^{L}+b} q^{-\frac{\left(\ell, M^{-1} \ell\right)}{4}} \prod_{i=1}^{L} x_{i}^{\ell_{i}}
$$

and "v.p." again means that we take principle value integral (i.e. take half-sum of contours $\left|z_{v}\right|=1 \pm \epsilon$ ). Such prescription corresponds to regularization by $\omega$ made in (A.16).

\section{B. Cabling in $M_{3}$ versus color in $T\left[M_{3}\right]$}

Here, we illustrate a delicate feature of $3 \mathrm{~d} / 3 \mathrm{~d}$ correspondence that shows up at the categorical level when knots are introduced in $M_{3}$. (The issue goes away if one looks at the problem without knots or at the decategorified level.) Namely, we compare $3 \mathrm{~d} \mathcal{N}=2$ theory $T\left[M_{3}\right]$ on $D^{2} \times S^{1}$ to quantum group invariants of $M_{3}$ and their categorification. As explained in section 4.1 and illustrated in Figure 1, introducing knots and links in $M_{3}$ corresponds to adding a line operator ("impurity") of a suitable type in $T\left[M_{3}\right]$.

A curious aspect of this correspondence is that the representation $\lambda$ ("color") of the line operator in $T\left[M_{3}\right]$ most naturally maps to the cabling data of the original knot $K$ in $M_{3}$. At the decategorified level, that data would be the same as the color of $K$ itself, but in the homological world the story is more interesting.
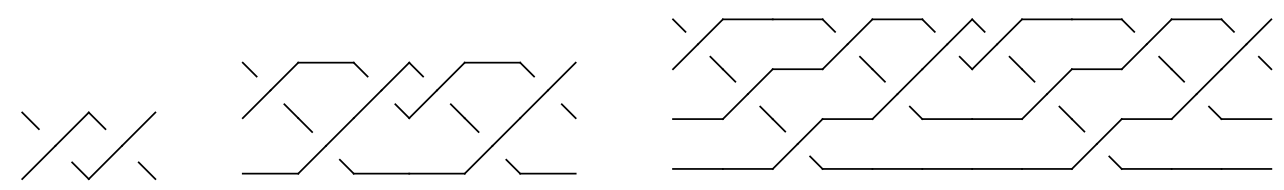

Figure 6: Braid representations of 2-, 3- and 4-cables of unknot with framing 1.

Since this phenomenon can be seen already in the basic case of the unknot, we illustrate it with the simplest choice of $G=S U(2), M_{3}=S^{3}$ and $K=$ unknot with framing 1 . Its 
colored Jones polynomials can be computed via the standard cabling formula that isolates an $n$-dimensional irreducible representation in the tensor product of 2-dimensional fundamental representations. As in [60], applying the same formula to the ordinary Khovanov homology of the $n$-cabling $K^{n}$ (illustrated in Figure 6):

$$
\begin{aligned}
& \mathcal{P}_{2}(\mathfrak{q}, t ; K)=\mathcal{P}_{1}\left(\mathfrak{q}, t ; K^{2}\right)-1 \\
& \mathcal{P}_{3}(\mathfrak{q}, t ; K)=\mathcal{P}_{1}\left(\mathfrak{q}, t ; K^{3}\right)-2 \mathcal{P}_{1}(\mathfrak{q}, t ; K) \\
& \mathcal{P}_{4}(\mathfrak{q}, t ; K)=\mathcal{P}_{1}\left(\mathfrak{q}, t ; K^{4}\right)-3 \mathcal{P}_{1}\left(\mathfrak{q}, t ; K^{2}\right)+1
\end{aligned}
$$

we find ${ }^{53}$

$$
\begin{aligned}
\mathcal{P}_{1}(\mathfrak{q},-t ; K)= & \mathfrak{q}^{5 / 2}+\sqrt{\mathfrak{q}} \\
\mathcal{P}_{2}(\mathfrak{q},-t ; K)= & \mathfrak{q}^{2}+\mathfrak{q}^{6} t^{2}+\mathfrak{q}^{4} t^{2} \\
\mathcal{P}_{3}(\mathfrak{q},-t ; K)= & \mathfrak{q}^{5 / 2}+\mathfrak{q}^{9 / 2}+\mathfrak{q}^{17 / 2} t^{4}+\mathfrak{q}^{21 / 2} t^{4}+\mathfrak{q}^{13 / 2} t^{2}+\mathfrak{q}^{5 / 2}(-t) \\
\mathcal{P}_{4}(\mathfrak{q},-t ; K)= & \mathfrak{q}^{16} t^{8}+\mathfrak{q}^{14} t^{8}+\mathfrak{q}^{12} t^{6}+\mathfrak{q}^{10} t^{4}+\mathfrak{q}^{4}\left(1-t^{3}\right)+\mathfrak{q}^{6}\left(t^{2}-t^{3}\right)+\mathfrak{q}^{8}\left(-t^{5}+t^{4}+t^{2}\right) \\
& \ldots
\end{aligned}
$$

where we changed $t \rightarrow-t$ in order to show explicitly the $\mathbb{Z}_{2}$ grading (by fermion number) via \pm signs. Note that the choice of framing played an important role in this calculation. In particular, if it was trivial, the result would be very different (even at the level of total dimensions) because the cables would be just disconnected unions of unknots, instead of non-trivial links shown in Figure 6.

Now, let us compare (B.2) with the expectation values (4.48) of the Wilson line operators in $3 \mathrm{~d} \mathcal{N}=2$ theory $T\left[S^{3}\right]$ :

$$
\begin{aligned}
& \left\langle W_{1}\right\rangle=t^{-1 / 4}\left(\mathfrak{q}^{5 / 2}+\sqrt{\mathfrak{q}}\right) \\
& \left\langle W_{2}\right\rangle=\mathfrak{q}^{2}+\frac{\mathfrak{q}^{6}}{t}+\frac{\mathfrak{q}^{4}}{t} \\
& \left\langle W_{3}\right\rangle=\mathfrak{q}^{5 / 2}+\mathfrak{q}^{9 / 2}+\frac{\mathfrak{q}^{17 / 2}}{t^{2}}+\frac{\mathfrak{q}^{21 / 2}}{t^{2}}-\frac{\mathfrak{q}^{5 / 2}}{t}+\frac{\mathfrak{q}^{13 / 2}}{t} \\
& \left\langle W_{4}\right\rangle=\frac{\mathfrak{q}^{16}}{t^{4}}+\frac{\mathfrak{q}^{14}}{t^{4}}+\frac{\mathfrak{q}^{12}}{t^{3}}+\frac{\mathfrak{q}^{10}}{t^{2}}+\mathfrak{q}^{6}\left(\frac{1}{t}-\frac{1}{t^{2}}\right)+\mathfrak{q}^{4}\left(1-\frac{1}{t^{2}}\right)+\mathfrak{q}^{8}\left(-\frac{1}{t^{3}}+\frac{1}{t^{2}}+\frac{1}{t}\right)
\end{aligned}
$$

where, as in section 4.2 , we made the replacement $q=\mathfrak{q}^{2} / t$. We find perfect agreement at the level of $\mathbb{Z} \times \mathbb{Z}_{2}$ graded spaces, where the first factor is the $\mathfrak{q}$-grading and the second factor is the fermion number grading. The $t$-gradings do not exactly agree (although they are somewhat correlated). This is a standard feature of the relation between colored knot homology and homology of cablings $[60,61]$.

The comparison between (B.2) and (B.3) clearly shows that the representation ("color") of the Wilson line operator in $T\left[S^{3}\right]$ appears to map to the cabling data of the original unknot in $M_{3}=S^{3}$. Note, a priori neither (B.2) nor (B.3) have any direct relation to the homology of the unknot colored by representation $\lambda$. Indeed, $\lambda$-colored homology of the unknot $[34,58]$

\footnotetext{
${ }^{53}$ using KnotTheory package for Mathematica
} 
should be obtained by using the categorified Jones-Wenzl projectors [71,72] instead of the naive cabling relations (B.1).

Similarly, (B.3) may miss the mark for two reasons. First, the variable $t$ that appears in (B.3) keeps track of the flavor symmetry grading, not the homological (R-symmetry) grading. This issue is not a serious objection, especially in view of the "homological-flavor locking" that allowed to identify these two gradings in many examples in this paper. A more serious issue with (B.3) has to do with a non-trivial map of line operators in $3 \mathrm{~d} / 3 \mathrm{~d}$ correspondence. In particular, the $n$-th cabling ${ }^{54}$ of a knot $K$ and its $n$-colored version may be indistinguishable at the decategorified level, but they are completely different operators in the physical setup (4.1) and, in general, have different homological invariants (spaces of BPS states).

\section{Categorification of the Turaev-Viro invariants}

The aim of this paper is to introduce and study new three-manifold invariants. Along the way, we have seen the important roles played by boundary conditions, abelian flat connections, homological blocks, refinement, and line operators. Here, we outline how these ideas can be applied to categorification of the Turaev-Viro invariants.

Defined via a state-sum model, the Turaev-Viro (TV) invariants [73] involve a "counting" problem from the start. This motivates a series of questions: do TV invariants of $M_{3}$ have a natural home in physics? Could it be that they are counting BPS states associated with the theory $T\left[M_{3}\right]$ and can be categorified in a way similar to the WRT invariant? How are they related to the new invariants discussed in this paper? Can they also be decomposed into the "atoms" of three-manifold invariants - the homological blocks and abelian connections? Can they be refined?

From the perspective of the state-sum model, categorification of the Turaev-Viro invariants may potentially seem even more natural than that of Chern-Simons (WRT) invariants, though the two problems are closely connected. Thus, the TV invariant of a closed 3-manifold $M_{3}$ is simply the square of the corresponding $S U(2)$ WRT/CS invariants [74-76]:

$$
\operatorname{TV}\left(M_{3}, q\right)=\left|Z^{\mathrm{CS}}\left(M_{3}, q\right)\right|^{2}
$$

As explained in detail in this paper, the WRT invariants can be written as a linear sum of homological blocks and, when there is only one block,

$$
\operatorname{TV}\left(M_{3}, q\right)=\widehat{Z}_{0}\left(M_{3}, q\right) \widehat{Z}_{0}\left(M_{3}, q^{-1}\right)=\mathcal{I}_{T\left[M_{3}\right]}(q)
$$

is exactly given by the index of the theory $T\left[M_{3}\right]$. So, for homological spheres, we have already categorified their TV invariants!

For general $M_{3}$, the story will be more interesting. ${ }^{55}$ If we use $V_{B}$ to denote the vector space of boundary conditions on $S^{1}$ of the theory $T\left[M_{3}\right]$ (a de-categorification of $\mathcal{C}_{B}$ ), then

\footnotetext{
${ }^{54}$ more precisely, the linear combination of cablings up to $n$-th, given by (B.1)

${ }^{55}$ To streamline the presentation, we will ignore normalization factors, Weyl group actions, etc.
} 
there are two natural bases $\{|\widehat{a}\rangle\}$ and $\{|a\rangle\}$ corresponding to the homological blocks and (connected components of) abelian flat connections. There are several distinct elements in $V_{B}$, one is $|0\rangle$ - the boundary condition at the origin of $D^{2}$, and another is $|\mathrm{CS}\rangle$ - the boundary condition at $\partial D^{2}$ that is used to reproduce the WRT invariant. Partition function of the theory $T\left[M_{3}\right]$ on $S^{1} \times I \times S^{1}$ defines the inner product on $V_{B}$ and a map $V_{B} \rightarrow V_{B}^{*}$. Then we have ${ }^{56}$

$$
\begin{aligned}
Z^{\mathrm{CS}}(q, t) & =\langle 0 \mid \mathrm{CS}\rangle, \\
Z_{a}(q, t) & =\langle 0 \mid a\rangle, \\
\widehat{Z}_{a}(q, t) & =\langle 0 \mid \widehat{a}\rangle, \\
\mathcal{I}(q, t) & =\langle 0 \mid 0\rangle,
\end{aligned}
$$

and also, in the unrefined limit,

$$
\mathrm{TV}(q)=Z^{\mathrm{CS}}(q) Z^{\mathrm{CS}}\left(q^{-1}\right)=\langle 0 \mid \mathrm{CS}\rangle\langle\mathrm{CS} \mid 0\rangle
$$

Using

$$
|\mathrm{CS}\rangle=\sum_{a} e^{2 \pi i k \operatorname{CS}(a)}|a\rangle
$$

we have

$$
|\mathrm{CS}\rangle\left\langle\mathrm{CS}\left|=\sum_{a, b} e^{2 \pi i k(\mathrm{CS}(a)-\mathrm{CS}(b))}\right| a\right\rangle\langle b|=\sum_{j} \mathcal{O}_{j}
$$

where

$$
\mathcal{O}_{j}=\sum_{a} \widetilde{q}^{\ell k(j, 2 a-j)}|a-j\rangle\langle a|,
$$

is the shift operator that acts on $Z_{a}$ by

$$
Z_{a} \mapsto \widetilde{q}^{\ell k(j, 2 a-j)} Z_{a-j}
$$

with

$$
\widetilde{q}=e^{-2 \pi i k}
$$

being the modular transform of $q$. The shift operators are the S-dual of Wilson loops, whose action on the homological block for $M_{3}=L(k, 1)$, as we have seen in section 4.3.1, is

$$
\widehat{Z}_{a} \mapsto q^{j(2 a-j)} \widehat{Z}_{a-j}
$$

The S-duality of type IIB string theory becomes 3d mirror symmetry of $T\left[M_{3}\right]$ that exchanges Wilson loops and vortex loops (see e.g. [77] for a detailed description of the map in a very large class of $3 \mathrm{~d} \mathcal{N}=4$ theories), and one expects that $\mathcal{O}_{j}$ corresponds to the insertion

\footnotetext{
${ }^{56}$ To obtain homological invariants, one just needs to decompactify the time $S^{1}$ circle, and $V_{B}$ will be come the category $\mathcal{C}_{B}$ that the $3 \mathrm{~d}$ theory $T\left[M_{3}\right]$ associates to $S^{1}$. Then the homological invariants are identified with $\operatorname{Hom}\left(B_{1}, B_{2}\right)$ between two boundary conditions $B_{1}, B_{2}$ in $\mathcal{C}_{B}$.
} 
of a vortex line. Similar to a Wilson loop, which carries an electric charge, a vortex loop carries a magnetic flux, creating a holonomy along its meridian — no surprise that it will shift $a$ which is characterized by holonomies. The relation between vortex loops and abelian flat connections can be understood more precisely using the M-theory geometry, as we now explain.

Vortex loops come from codimension-two defects in $6 \mathrm{~d}(2,0)$ theory and can be engineered by a stacks of "defect M5-branes" as follows:

$\begin{array}{cccc}\text { M5-branes: } & \mathbb{R} \times M_{3} \times & D^{2} \\ & \cap & \cap \\ \text { space-time: } & \mathbb{R} \times T^{*} M_{3} \times & T N, \\ & & \cup & \cup \\ \text { "defect" M5'-branes: } & \mathbb{R} \times & M_{3} \times\left. T^{*}\right|_{O}\end{array}$

where $\left.T^{*}\right|_{O}$ is the cotangent space at $O$. The defect fivebranes have a flat background 2form connection ("gerbe connection") on its world volume. The vortex lines will carry fluxes, labeled by elements in $H_{1}\left(M_{3}, \mathbb{Z}\right)^{*}$. Analogous to the BPS state counting analysis with M2branes, the non-torsion part of $H_{1}\left(M_{3}, \mathbb{Z}\right)^{*}$ results in $Q$-exact deformations of the system, enabling us to consider only the torsion part (Tor $\left.H_{1}\left(M_{3}, \mathbb{Z}\right)\right)^{*}$. Taking into account multiple fivebranes gives (Tor $\left.H_{1}\left(M_{3}, \Lambda_{\mathrm{wt}, \mathfrak{g}}\right)\right)^{*} / W_{G}$. In other words, non-trivial BPS vortex loop are labeled by components of abelian flat connections. For M2-branes propagating in this system (C.14), this generates the above mentioned shift action of vortex loops on flat connections.

We have now identified the system relevant for the Turaev-Viro invariant: $T\left[M_{3}\right]$ on $\mathbb{R} \times S^{2}$ with the insertion of a exotic defect, given by a linear combination of vortex loops, ${ }^{57}$ whose BPS Hilbert space should categorify the TV invariant. This Hilbert space will be doubly-graded for generic $M_{3}$ and at least triply-graded for Seifert $M_{3}$. In the latter case, we will have refinement for both TV invariants and the "TV homologies." It would be interesting to test this proposal in concrete examples, which we leave for future work.

\section{References}

[1] P. Kronheimer and T. Mrowka, Monopoles and three-manifolds, vol. 10. Cambridge University Press, 2007.

[2] A. Floer, An instanton-invariant for 3-manifolds, Comm. Math. Phys. 118 (1988) 215-240.

[3] P. Ozsváth and Z. Szabó, Holomorphic disks and topological invariants for closed three-manifolds, Annals of Mathematics (2004) 1027-1158.

[4] E. Witten, Quantum Field Theory and the Jones Polynomial, Commun. Math. Phys. 121 (1989) 351-399.

[5] N. Reshetikhin and V. G. Turaev, Invariants of 3-manifolds via link polynomials and quantum groups, Invent. Math. 103 (1991) 547-597.

\footnotetext{
${ }^{57}$ This defect can be viewed as the S-dual of a Wilson loop labeled by a reducible representation, but it will be interesting to find an alternative way to characterize this defect.
} 
[6] D. Birmingham, M. Blau, M. Rakowski and G. Thompson, Topological field theory, Phys. Rept. 209 (1991) 129-340.

[7] L. Rozansky and H. Saleur, $S$ and T matrices for the superU(1,1) WZW model: Application to surgery and three manifolds invariants based on the Alexander-Conway polynomial, Nucl. Phys. B389 (1993) 365-423, [hep-th/9203069].

[8] D. Chang, I. Phillips and L. Rozansky, $R$ matrix approach to quantum superalgebras su- $q(\mathrm{~m} / \mathrm{n})$, J. Math. Phys. 33 (1992) 3710-3715, [hep-th/9207075].

[9] L. Rozansky and H. Saleur, Reidemeister torsion, the Alexander polynomial and U(1,1) Chern-Simons Theory, J. Geom. Phys. 13 (1994) 105-123, [hep-th/9209073].

[10] G. Meng and C. H. Taubes, Sw= milnor torsion, Mathematical Research Letters 3 (1996) 661-674.

[11] L. Crane and I. B. Frenkel, Four-dimensional topological quantum field theory, Hopf categories, and the canonical bases, J. Math. Phys. 35 (1994) 5136-5154.

[12] M. Khovanov, A categorification of the jones polynomial, arXiv preprint math.QA/9908171 (1999) .

[13] M. Khovanov and L. Rozansky, Matrix factorizations and link homology, Fundamenta Mathematicae 199 (2008) 1-91.

[14] I. Frenkel, C. Stroppel and J. Sussan, Categorifying fractional Euler characteristics, Jones-Wenzl projectors and 3j-symbols, Quantum Topol. 3 (2012) 181-253.

[15] H. Ooguri and C. Vafa, Knot invariants and topological strings, Nucl. Phys. B577 (2000) 419-438, [hep-th/9912123].

[16] S. Gukov, A. S. Schwarz and C. Vafa, Khovanov-Rozansky homology and topological strings, Lett. Math. Phys. 74 (2005) 53-74, [hep-th/0412243].

[17] S. Gukov, Gauge theory and knot homologies, Fortsch. Phys. 55 (2007) 473-490, [0706.2369].

[18] E. Witten, Fivebranes and Knots, 1101.3216.

[19] M. Aganagic and S. Shakirov, Knot Homology and Refined Chern-Simons Index, Commun. Math. Phys. 333 (2015) 187-228, [1105.5117].

[20] S. Chun, S. Gukov and D. Roggenkamp, Junctions of surface operators and categorification of quantum groups, 1507.06318.

[21] S. Nawata and A. Oblomkov, Lectures on knot homology, 2015. 1510.01795.

[22] S. Gukov, M. Marino and P. Putrov, Resurgence in complex Chern-Simons theory, 1605.07615.

[23] S. Cecotti and C. Vafa, Topological antitopological fusion, Nucl. Phys. B367 (1991) 359-461.

[24] C. Beem, T. Dimofte and S. Pasquetti, Holomorphic Blocks in Three Dimensions, JHEP 12 (2014) 177, [1211.1986].

[25] S. Cecotti, D. Gaiotto and C. Vafa, $t t^{*}$ geometry in 3 and 4 dimensions, JHEP 05 (2014) 055, [1312.1008].

[26] S. Gukov, P. Putrov and C. Vafa, Fivebranes and 3-manifold homology, 1602.05302. 
[27] K. Hikami, Decomposition of witten-reshetikhin-turaev invariant: linking pairing and modular forms, Chern-Simons Gauge Theory 20 (2011) 131-151.

[28] M. Atiyah, On framings of 3-manifolds, Topology 29 (1990) 1-7.

[29] Y. Yoshida and K. Sugiyama, Localization of $3 d \mathcal{N}=2$ Supersymmetric Theories on $S^{1} \times D^{2}$, 1409.6713.

[30] S. Pasquetti, Factorisation of $N=2$ Theories on the Squashed 3-Sphere, JHEP 04 (2012) 120, [1111.6905].

[31] M. Blau and G. Thompson, Chern-Simons Theory with Complex Gauge Group on Seifert Fibred 3-Manifolds, 1603.01149.

[32] F. Benini and A. Zaffaroni, A topologically twisted index for three-dimensional supersymmetric theories, JHEP 07 (2015) 127, [1504.03698].

[33] J. M. F. Labastida, M. Marino and C. Vafa, Knots, links and branes at large N, JHEP 11 (2000) 007, [hep-th/0010102].

[34] E. Gorsky, S. Gukov and M. Stosic, Quadruply-graded colored homology of knots, 1304.3481.

[35] M. Aganagic, T. Ekholm, L. Ng and C. Vafa, Topological Strings, D-Model, and Knot Contact Homology, Adv. Theor. Math. Phys. 18 (2014) 827-956, [1304.5778].

[36] V. Shende, D. Treumann and E. Zaslow, Legendrian knots and constructible sheaves, 1402.0490.

[37] V. Shende, D. Treumann, H. Williams and E. Zaslow, Cluster varieties from Legendrian knots, 1512.08942.

[38] S. Gukov and D. Pei, Equivariant Verlinde formula from fivebranes and vortices, 1501.01310.

[39] D. Jafferis and X. Yin, A Duality Appetizer, 1103.5700.

[40] A. Kapustin, H. Kim and J. Park, Dualities for 3d Theories with Tensor Matter, JHEP 12 (2011) 087, [1110.2547].

[41] D. Pei and K. Ye, A 3d-3d appetizer, JHEP 11 (2016) 008, [1503.04809].

[42] S. Gukov, S. Nawata, I. Saberi, M. Stoi and P. Sukowski, Sequencing BPS Spectra, JHEP 03 (2016) 004, [1512.07883].

[43] C. Beem, M. Lemos, P. Liendo, W. Peelaers, L. Rastelli and B. C. van Rees, Infinite Chiral Symmetry in Four Dimensions, Commun. Math. Phys. 336 (2015) 1359-1433, [1312.5344].

[44] M. Dedushenko, S. S. Pufu and R. Yacoby, A one-dimensional theory for Higgs branch operators, 1610.00740 .

[45] A. Gadde, S. Gukov and P. Putrov, Fivebranes and 4-manifolds, 1306.4320.

[46] H.-J. Chung, T. Dimofte, S. Gukov and P. Sukowski, 3d-3d Correspondence Revisited, JHEP 04 (2016) 140, [1405.3663].

[47] Y. Imamura and S. Yokoyama, Index for three dimensional superconformal field theories with general R-charge assignments, JHEP 04 (2011) 007, [1101.0557].

[48] S. Gukov, D. Pei, W. Yan and K. Ye, Equivariant Verlinde algebra from superconformal index and Argyres-Seiberg duality, 1605.06528. 
[49] J. E. Andersen, S. Gukov and D. Pei, The Verlinde formula for Higgs bundles, 1608.01761.

[50] D. Halpern-Leistner, The equivariant Verlinde formula on the moduli of Higgs bundles, ArXiv e-prints (Aug., 2016), [1608.01754].

[51] I. Cherednik, Jones polynomials of torus knots via DAHA, Int.Math.Res.Not. 23 (2013) 5366-5425, [1111.6195].

[52] F. Benini, Y. Tachikawa and D. Xie, Mirrors of 3d Sicilian theories, JHEP 09 (2010) 063, [1007.0992].

[53] D. Gang, E. Koh and K. Lee, Superconformal Index with Duality Domain Wall, JHEP 10 (2012) 187, [1205.0069].

[54] D. Gang, E. Koh, S. Lee and J. Park, Superconformal Index and 3d-3d Correspondence for Mapping Cylinder/Torus, JHEP 01 (2014) 063, [1305.0937].

[55] T. Dimofte, D. Gaiotto and S. Gukov, Gauge Theories Labelled by Three-Manifolds, Commun. Math. Phys. 325 (2014) 367-419, [1108.4389].

[56] T. Dimofte, D. Gaiotto and S. Gukov, 3-Manifolds and 3d Indices, Adv. Theor. Math. Phys. 17 (2013) 975-1076, [1112.5179].

[57] D. Gang, N. Kim, M. Romo and M. Yamazaki, Taming supersymmetric defects in 3d3d correspondence, J. Phys. A49 (2016) 30LT02, [1510.03884].

[58] S. Gukov and M. Stosic, Homological Algebra of Knots and BPS States, Proc. Symp. Pure Math. 85 (2012) 125-172, [1112.0030].

[59] H. Awata, S. Gukov, P. Sulkowski and H. Fuji, Volume Conjecture: Refined and Categorified, Adv. Theor. Math. Phys. 16 (2012) 1669-1777, [1203.2182].

[60] M. Khovanov, Categorifications of the colored jones polynomial, Journal of Knot Theory and Its Ramifications 14 (2005) 111-130.

[61] S. Gukov and J. Walcher, Matrix factorizations and Kauffman homology, hep-th/0512298.

[62] L. Rozansky, A categorification of the stable su (2) witten-reshetikhin-turaev invariant of links in s2 $x$ s1, arXiv preprint arXiv:1011.1958 (2010) .

[63] H. Itoyama, A. Mironov, A. Morozov and A. Morozov, HOMFLY and superpolynomials for figure eight knot in all symmetric and antisymmetric representations, JHEP 07 (2012) 131, [1203.5978].

[64] P. Wedrich, Exponential growth of colored HOMFLY-PT homology, 1602.02769.

[65] Ya. Kononov and A. Morozov, Rectangular superpolynomials for the figure-eight knot, 1609.00143.

[66] N. Reshetikhin and V. G. Turaev, Invariants of 3-manifolds via link polynomials and quantum groups, Inventiones mathematicae 103 (1991) 547-597.

[67] E. Witten, Quantum field theory and the jones polynomial, Communications in Mathematical Physics 121 (1989) 351-399.

[68] F. Deloup and V. Turaev, On reciprocity, Journal of Pure and Applied Algebra 208 (2007) $153-158$. 
[69] L. C. Jeffrey, Chern-simons-witten invariants of lens spaces and torus bundles, and the semiclassical approximation, Communications in mathematical physics 147 (1992) 563-604.

[70] R. Lawrence and D. Zagier, Modular forms and quantum invariants of 3-manifolds, Asian Journal of Mathematics 3 (1999) 93-108.

[71] B. Cooper and V. Krushkal, Categorification of the Jones-Wenzl projectors, Quantum Topol. 3 (2012) 139-180.

[72] B. Cooper, M. Hogancamp and V. Krushkal, SO(3) homology of graphs and links, Algebr. Geom. Topol. 11 (2011) 2137-2166.

[73] V. Turaev and O. Viro, State sum invariants of 3-manifolds and quantum 6j-symbols, Topology 31 (1992) $865-902$.

[74] V. G. Turaev et al., Shadow links and face models of statistical mechanics, J. Differential Geom 36 (1992) 35-74.

[75] K. Walker, On wittens 3-manifold invariants, preprint (1991) .

[76] V.-G. Turaev, Quantum invariants of knots and 3-manifolds. Walter de Gruyter \&Co., 1994.

[77] B. Assel and J. Gomis, Mirror Symmetry And Loop Operators, JHEP 11 (2015) 055, [1506.01718]. 